Merci d'utiliser le titre suivant lorsque vous citez ce document :

MacLeod, M. et al. (2015-08-01), « Rapport coût efficacité des mesures d'atténuation des émissions de gaz à effet de serre en agriculture : Examen des travaux publiés », Éditions OCDE, Paris.

http://dx.doi.org/10.1787/5jrvvkhfn7nr-fr

\title{
Rapport coût efficacité des mesures d'atténuation des émissions de gaz à effet de serre en agriculture
}

\author{
EXAMEN DES TRAVAUX PUBLIÉS
}

Michael MacLeod,

Vera Eory,

Guillaume Gruère,

Jussi Lankoski

La version originale de ce document a été publiée comme suit :

MacLeod, M. et al. (2015-08-01), "Cost-Effectiveness of Greenhouse Gas Mitigation Measures for Agriculture: A Literature Review", OECD Food, Agriculture and Fisheries Papers, No. 89, OECD Publishing, Paris. http://dx.doi.org/10.1787/5jrvvkq900vj-en 


\section{DOCUMENTS DE L'OCDE SUR L'ALIMENTATION, L'AGRICULTURE ET LA PÊCHERIE}

Ce document est publié sous la responsabilité du Secrétariat général de l'OCDE. Les opinions et les interprétations exprimées ne reflètent pas nécessairement les vue de l'OCDE ou des gouvernements de ses pays membres.

Ce document et toute carte qu'il peut comprendre sont sans préjudice du statut de tout territoire, de la souveraineté s'exerçant sur ce dernier, du tracé des frontières et limites internationales, et du nom de tout territoire, ville ou région.

La publication de ce document a reçu l'approbation de Ken Ash, Directeur, Direction des Échanges et de l'agriculture à l'OCDE.

Des commentaires sur la série sont les bienvenus et peuvent être envoyés à tad.contact@oecd.org.

\footnotetext{
Vous êtes autorisés à copier, télécharger ou imprimer du contenu OCDE pour votre utilisation personnelle. Vous pouvez inclure des extraits des publications, des bases de données et produits multimédia de I'OCDE dans vos documents, présentations, blogs, sites Internet et matériel d'enseignement, sous réserve de faire mention de la source OCDE et du copyright. Les demandes pour usage commercial ou de traduction devront être adressées à rights@oecd.org.
} 


\title{
Résumé \\ RAPPORT COÛT EFFICACITÉ DES MESURES D'ATTÉNUATION DES ÉMISSIONS DE GAZ À EFFET DE SERRE EN AGRICULTURE : EXAMEN DES TRAVAUX PUBLIÉS
}

\author{
Michael MacLeod, Vera Eory, \\ Guillaume Gruère et Jussi Lankoski
}

\begin{abstract}
Ce document livre un examen des travaux publiés à l'échelle internationale sur le rapport coûtefficacité des mesures axées sur l'offre qui permettent de réduire l'intensité des émissions de gaz à effet de serre agricoles en préservant ou renforçant la production. La présente étude analyse 65 études internationales récentes consacrées au rapport coût-efficacité de mesures agricoles d'atténuation, qui couvrent 181 activités différentes. Neuf études de cas de mesures d'atténuation largement appliquées illustrent, en général à partir d'une méthode d'estimation ascendante des coûts, les différences significatives du rapport coût-efficacité des mesures entre les pays et les études, dues en partie à des différences contextuelles. Les comparaisons entre ces études hétérogènes doivent être appréhendées avec toute la circonspection voulue, mais les résultats suggèrent que les mesures reposant sur l'efficience d'utilisation des engrais et l'amélioration génétique du bétail, ainsi que les possibilités d'amélioration de l'efficacité énergétique des engins et machines agricoles, sont considérées comme des solutions d'atténuation hautement performantes en terme de coût-efficacité dans les différents pays. Un premier examen des mesures publiques montre qu'il existe différentes solutions possibles pour encourager l'adoption de mesures économes et efficaces, allant de campagnes d'information à des stratégies d'incitation. Il convient d'approfondir l'analyse pour surmonter les difficultés d'estimation restantes et trouver comment les mesures d'atténuation peuvent s'inscrire dans le cadre plus vaste des stratégies climatiques, agricoles et environnementales.
\end{abstract}

Mots-clés : Agriculture et changement climatique, atténuation des émissions de gaz à effet de serre, coûtefficacité, Coût d'abattement marginal, estimation ascendante des coûts.

JEL : Q16, Q52, Q54, Q58.

\section{Remerciements}

Ce rapport a été écrit par Michael MacLeod et Vera Eory, du Scotland's Rural College, Edimbourg, Royaume Uni, et par Guillaume Gruère et Jussi Lankoski de l'OCDE. Le rapport a bénéficié du soutien financier du Programme pour la modification de l'environnement (2011-2016) de la Division des services scientifiques et analytiques liés au milieu rural et à l'environnement (RESAS) du Gouvernement écossais. Ils remercient aussi les délégations de l'OCDE, notamment les délégations française et irlandaise pour avoir transmis des publications et informations utiles. Les auteurs remercient aussi Ada Ignaciuk et Franck Jésus pour leurs commentaires sur des versions antérieures de ce rapport. Ils remercient enfin Michèle Patterson pour avoir supporté la préparation de cette publication. 


\section{Table des matières}

Résumé.

1. L'atténuation des émissions agricoles de gaz à effet de serre : gros plan sur l'amélioration de l'intensité GES du côté de l'offre

2. Trois grandes méthodes d'évaluation des coûts des mesures d'atténuation : avantages et inconvénients.

3. Étendue des publications analysées : 65 études fournissent une estimation du rapport coûtefficacité de pas moins de 181 mesures d'atténuation des émissions agricoles de GES

4. Études de cas de mesures spécifiques : des coûts très divers ............................................................. 21

5. Des mesures d'atténuation aux stratégies d'atténuation possibles : débat préliminaire 44

6. Principaux enseignements : quelques mesures notables, beaucoup d'autres de rapport coûtefficacité variable et travaux de recherche nécessaires sur les estimations et les solutions possibles à l'appui de l'application..

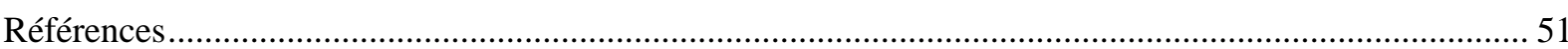

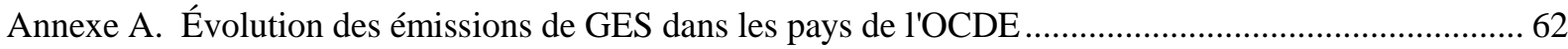

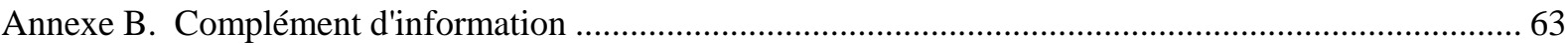

\section{Tableaux}

Tableau 1. Nombre de mesures d'atténuation de chaque catégorie et nombre total de reprises des mesures d'atténuation de chaque catégorie dans les études analysées ...........................20

Tableau 2. Principales sources d'émissions au niveau de l'exploitation visées par les mesures d'atténuation des études de cas.......................................................................................22

Tableau 3. Réduction des émissions associée aux cultures de couverture semées pendant la période de jachère de la rotation des grandes cultures ....................................................24

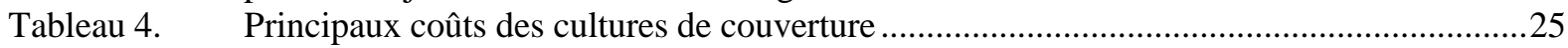

Tableau 5. Taux de réduction des émissions pour l'apport raisonné de nutrients .................................27

Tableau 6. Estimations du rapport coût-efficacité pour l'apport raisonné de nutrients .........................27

Tableau 7. Taux de réduction des émissions associé aux inhibiteurs de nitrification...........................29

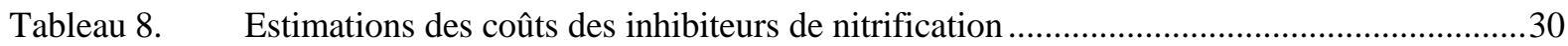

Tableau 9. Rapport coût-efficacité estimé des inhibiteurs de nitrification ..........................................30

Tableau 10. Réduction des émissions associé au boisement des pâturages ............................................31

Tableau 11. Principaux coûts et avantages ponctuels et récurrents du boisement des pâturages ............32

Tableau 12. Estimations des coûts et avantages du boisement des pâturages ..........................................32

Tableau 13. Rapport coût-efficacité estimé du boisement des pâturages ................................................32

Tableau 14. Réduction des émissions associée à l'élévation de la teneur en matières grasses de

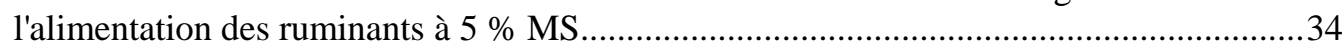

Tableau 15. Estimations du rapport coût-efficacité de la complémentation en lipide dans

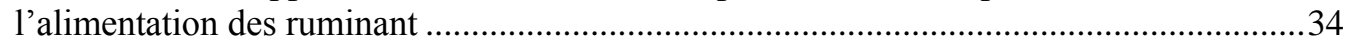

Tableau 16. Réduction des émissions avec l'amélioration génétique pour réduire les émissions de $\mathrm{CH} 4$ liées à la fermentation entérique. 
Tableau 17. Estimations du rapport coût-efficacité avec l'amélioration génétique pour réduire les émissions de $\mathrm{CH} 4$ liées à la fermentation entérique......................................................37

Tableau 18. Réduction des émissions avec la digestion anaérobie.... 37

Tableau 19. Estimations du rapport coût-efficacité avec la digestion anaérobie

Tableau 20. Économies moyennes de carburant réalisables en modifiant l'utilisation des engins agricoles, selon la base de données Efficient 20 (Lieu : UE)

Tableau 21. Coûts et économies récurrents associés à l'amélioration de l'efficacité énergétique des engins agricoles $(+:$ coût ; - : économie)

Tableau 22. Réduction des émissions associée à l'introduction de légumineuses dans les systèmes de cultures et les pâturages .....

Tableau 23. Coûts ou économies avec la fixation biologique de l'azote

Tableau 24. Source possible d'incommensurabilité des études

Tableau 25. Exemples de stratégies d'atténuation des GES

Tableau 26.

Exemples de coûts (-) et avantages (+) secondaires de certaines mesures courantes d'atténuation des GES

Tableau 27. Études récentes du rapport coût-efficacité de mesures multiples d'atténuation des émissions de GES .....

Tableau 28. Synthèse des sous-catégories de mesures d'atténuation incluses dans chaque étude (pour les études comportant un calcul du rapport coût-efficacité)

Tableau 29. Nombre de mesures d'atténuation dans chaque catégorie (en gras) et souscatégorie (en italique)

Tableau 30. Nombre de cas où chaque mesure d'atténuation apparaît dans les études analysées

\section{Graphiques}

Graphique 1. Principales sources et principaux processus d'émission et d'absorption de gaz à effet de serre dans les exploitations agricoles des écosystèmes gérés

Graphique 2. Évolution des émissions de gaz à effet de serre, de la valeur ajoutée agricole et de la productivité agricole dans les pays de l'OCDE (1990-2010)...................................

Graphique 3. Coûts et avantages marginaux de réduction des émissions.................................................12

Graphique 4. Exemple de courbe du coût marginal de réduction des émissions (MACC) associée aux mesures d'atténuation des émissions de GES du secteur laitier britannique .................13

Graphique 5. MACC projetée des émissions de GES non-CO2 pour l'agriculture

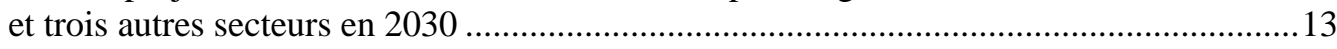

Graphique 6. Classification des coûts engendrés par l'atténuation des émissions de GES ......................16

Graphique 7. Estimations minimales et maximales du rapport coût-efficacité (EUR/tCO2e) des mesures d'atténuation envisagées dans 12 études de MACC .......................................46

Graphique 8. Émissions de gaz à effet de serre $\left(\mathrm{CH}_{4}\right.$ et $\left.\mathrm{N}_{2} \mathrm{O}\right)$ d'origine agricole, pays de l'OCDE, 1990-2010

\section{Encadrés}

Encadré 1. Atténuation des émissions dans une agriculture fondée sur l'élevage de ruminants le cas de l'Irlande.

Encadré 2. L'utilisation de MACCs pour analyser les rapports coût-efficacité projetés des options de réduction des émissions par secteur et pays .............................................13

Encadré 3. Implications des différentes approches modélisatrices : résultats d'une méta-analyse ........16

Encadré 4. Les MACC dans le cas de polluants multiples

Encadré 5. Exemples de mesures d'atténuation alternatives

Encadré 6.

Potentiel d'atténuation et coût de dix actions techniques : le cas de la France 


\section{RÉSUMÉ}

Les émissions agricoles de gaz à effet de serre (GES) représentent une part non négligeable du total des émissions des pays de l'OCDE. Sur la période 2008-10, les GES d'origine agricole émis au niveau de l'exploitation (hors émissions en amont et en aval de l'exploitation, consommation d'énergie dans les exploitations et changement d'affectation des terres) ont représenté $8 \%$ du total des émissions des pays de l'OCDE notifiées au titre de la Convention-cadre des Nations Unies sur les changements climatiques.

L'agriculture peut avoir un rôle à jouer dans l'atténuation des émissions de GES malgré la hausse de la demande alimentaire à laquelle elle doit faire face. La demande des principaux produits agroalimentaires devrait connaître une croissance soutenue d'ici à 2050 ; dans un tel contexte, les objectifs de production agricole devront être réalisés tout en cherchant à faire baisser le total mondial des émissions de GES. Il est tout à fait possible, dans les pays de l'OCDE, de réduire l'intensité d'émission (émissions par unité produite) de l'agriculture tout en améliorant la productivité. En fonction du taux de croissance de la productivité, cette réduction de l'intensité d'émission pourrait même déboucher sur une diminution absolue des émissions.

Dans les pays de l'OCDE, l'intensité d'émission de la production agricole a pu être sensiblement améliorée grâce aux évolutions techniques et aux mesures prises par les pouvoirs publics. Selon les estimations disponibles, les pays de l'OCDE ont réduit l'intensité de leurs émissions agricoles d'environ $2 \%$ par an en moyenne entre 2000 et 2010. Ce résultat s'explique par l'adoption conjointe de techniques et de pratiques de gestion agricole plus efficaces et par la mise en œuvre d'incitations à la réduction des émissions, à la faveur des diverses stratégies et réformes engagées dans les pays de l'OCDE.

Pour aller plus loin, il est nécessaire de connaître les différentes solutions envisageables pour atténuer les émissions de GES d'origine agricole et de déterminer si elles sont techniquement efficaces, économiquement efficientes et acceptables pour la société. En particulier, le rapport coût-efficacité des mesures d'atténuation dans l'agriculture, qui a fait l'objet de multiples études, est souvent très variable, parce qu'il dépend de la situation géographique, des conditions climatiques et des pratiques agricoles passées et actuelles; il reste par conséquent difficile à mesurer et à évaluer pour étayer l'action des pouvoirs publics.

Le présent document livre un examen des travaux publiés à l'échelle internationale sur le rapport coûtefficacité des mesures axées sur l'offre qui permettent de réduire l'intensité des émissions en préservant ou renforçant la production. Par conséquent, il n'analyse pas les solutions axées sur la demande : il se concentre sur les pratiques agricoles d'atténuation plutôt que sur les moyens d'intervention possibles (tels que la taxe carbone ou l'échange de droits d'émission) et privilégie les pratiques permettant de réduire les émissions tout en préservant ou en renforçant la production. Il ne s'agit pas d'une méta-analyse, en ce sens qu'il ne prétend pas évaluer le rapport coût-efficacité moyen des mesures.

Il existe de très nombreuses mesures possibles d'atténuation et d'importantes variations entre les estimation sde de leurs impact, partiellement dû à des spécificités méthodologiques. Différentes méthodes peuvent permettre de dériver les courbes du coût marginal de réduction des émissions : i) l'estimation ascendante des coûts; ii) la modélisation microéconomique, en fonction de prix exogènes; iii) les modèles d'équilibre de l'offre par région ou par secteur. La présente étude identifie et analyse 65 études internationales récentes consacrées au rapport coût-efficacité de huit catégories de mesures agricoles d'atténuation, qui couvrent 181 activités différentes. Neuf études de cas de mesures d'atténuation largement appliquées illustrent, en général à partir d'une méthode d'estimation ascendante des coûts, les 
différences significatives du rapport coût-efficacité des mesures entre les pays et les études, dues en partie à des différences contextuelles.

Les comparaisons entre ces études hétérogènes doivent être appréhendées avec toute la circonspection voulue, mais les résultats suggèrent que les mesures reposant sur l'efficience d'utilisation des engrais et l'amélioration génétique du bétail, ainsi que les possibilités d'amélioration de l'efficacité énergétique des engins et machines agricoles, sont considérées comme des solutions d'atténuation hautement performantes en terme de coût-efficacité dans les différents pays.

Un premier examen des mesures publiques montre qu'il existe différentes solutions possibles pour encourager l'adoption de mesures économes et efficaces, allant de campagnes d'information à des stratégies d'incitation. L'adoption des mesures les plus performantes à faible coût (ou à coût négatif) devrait pouvoir être encouragée par des stratégies axées sur l'éducation et l'information. S'agissant des mesures susceptibles d'entraîner un coût positif ou modéré mais procurant des avantages nets à la société (coût de mise en œuvre inférieur au coût social des émissions), le recours à des incitations pourrait être justifié. Dans ce cas, deux types de démarche peuvent être envisagés, l'une basée sur l'utilisation d'instruments économiques sectoriels ou plus généraux (taxe ou plafonnement et échange, par exemple), l'autre, sur des paiements volontaires visant à promouvoir des mesures d'atténuation ciblées : toutes deux peuvent présenter des avantages et des inconvénients.

Il convient d'approfondir l'analyse pour surmonter les difficultés d'estimation restantes et trouver comment les mesures d'atténuation peuvent s'inscrire dans le cadre plus vaste des stratégies climatiques, agricoles et environnementales. Malgré le nombre croissant de travaux empiriques, la mesure du rapport coût-efficacité se heurte toujours à plusieurs problèmes techniques, notamment pour : a) prévoir le rapport coût-efficacité des différentes combinaisons de mesures; b) tenir compte des fuites et des effets de déplacement qui transfèrent les émissions d'un lieu à un autre ; c) rendre compte de la variabilité et de l'incertitude du rapport coût-efficacité ; d) comprendre pourquoi les agriculteurs sont parfois réticents à adopter des mesures d'atténuation a priori «doublement gagnantes». Une analyse exhaustive serait également nécessaire pour évaluer les coûts et les avantages des différents leviers de la réduction des émissions de GES d'origine agricole. 


\section{L'atténuation des émissions agricoles de gaz à effet de serre : gros plan sur l'amélioration de l'intensité GES du côté de l'offre}

Les émissions de gaz à effet de serre (GES) d'origine agricole représentent une part non négligeable du total des émissions des pays de l'OCDE. Sur la période 2008-10, l'agriculture a été responsable de $8 \%$ du total des émissions de l'OCDE comptabilisé par la Convention-cadre des Nations Unies sur les changements climatiques (CCCC) (consommation d'énergie et changement d'affectation des terres non compris) (OCDE, 2013, voir aussi le graphique 8 en annexe).

Comme l'illustre le graphique 1, les principaux GES associés à la production agricole sont les suivants :

- le méthane $\left(\mathrm{CH}_{4}\right)$ issu principalement de la décomposition de matière organique dans des conditions anaérobies dans le cadre de la fermentation entérique et de la gestion du fumier, mais aussi de la riziculture ;

- le protoxyde d'azote $\left(\mathrm{N}_{2} \mathrm{O}\right)$ issu de la transformation microbienne de l'azote $(\mathrm{N})$ dans les sols et les fumiers - lors de l'épandage d'engrais organiques ou de synthèse et par l'intermédiaire de l'urine et des déjections du bétail ;

- le dioxyde de carbone $\left(\mathrm{CO}_{2}\right)$ issu de a) la consommation d'énergie au niveau de l'exploitation, en amont et en aval, et b) des variations des stocks de carbone dans la biomasse aérienne et dans le sol liées à l'affectation des terres et à son changement.

Les émissions agricoles annuelles de $\mathrm{CH}_{4}$ et de $\mathrm{N}_{2} \mathrm{O}$ des pays de l'OCDE au niveau de l'exploitation ont atteint 1183 milliards de tonnes d'équivalent $\mathrm{CO}_{2}\left(\mathrm{CO}_{2} \mathrm{e}\right)$ sur la période 2008-10(OCDE, 2013 : p. 144), réparties à part égales entre les deux gaz. Parallèlement, la proportion des émissions agricoles dans le total de chaque pays a présenté de fortes disparités, allant de $2 \%$ au Japon à $46 \%$ en NouvelleZélande (voir les tendances et estimations détaillées par pays à l'annexe A). Ces chiffres ne comprennent pas les émissions de $\mathrm{CO}_{2}$ résultant de la combustion des combustibles et carburants sur l'exploitation (pour les travaux agricoles, par exemple), du changement d'affectation des terres au niveau des exploitations et des activités hors exploitation telles que la production d'intrants (aliments du bétail, énergie et engrais notamment).

Compte tenu de cette part de responsabilité, un certain nombre de rapports et d'initiatives ont mis en avant le potentiel de l'agriculture dans l'atténuation des émissions de GES (Smith et al., 2014, par exemple). La demande des principaux produits agroalimentaires devrait connaître une croissance soutenue d'ici à 2050; dans un tel contexte, l'abaissement du total mondial des émissions de GES par le ralentissement de la production agricole des pays de l'OCDE est de moins en moins probable ${ }^{1}$. En revanche, il est possible de réduire l'intensité d'émission (à savoir, les émissions par unité produite) de l'agriculture dans les pays de l'OCDE tout en améliorant la productivité.

1. Encore qu'une rationalisation soit sans doute possible par un transfert de la production actuelle, soit des pays de l'OCDE vers d'autres pays membres, soit des pays de l'OCDE vers des pays non-membres dont les conditions climatiques ou pédologiques peuvent être plus adaptées à une production agricole plus efficiente. 
Graphique 1. Principales sources et principaux processus d'émission et d'absorption de gaz à effet de serre dans les exploitations agricoles des écosystèmes gérés

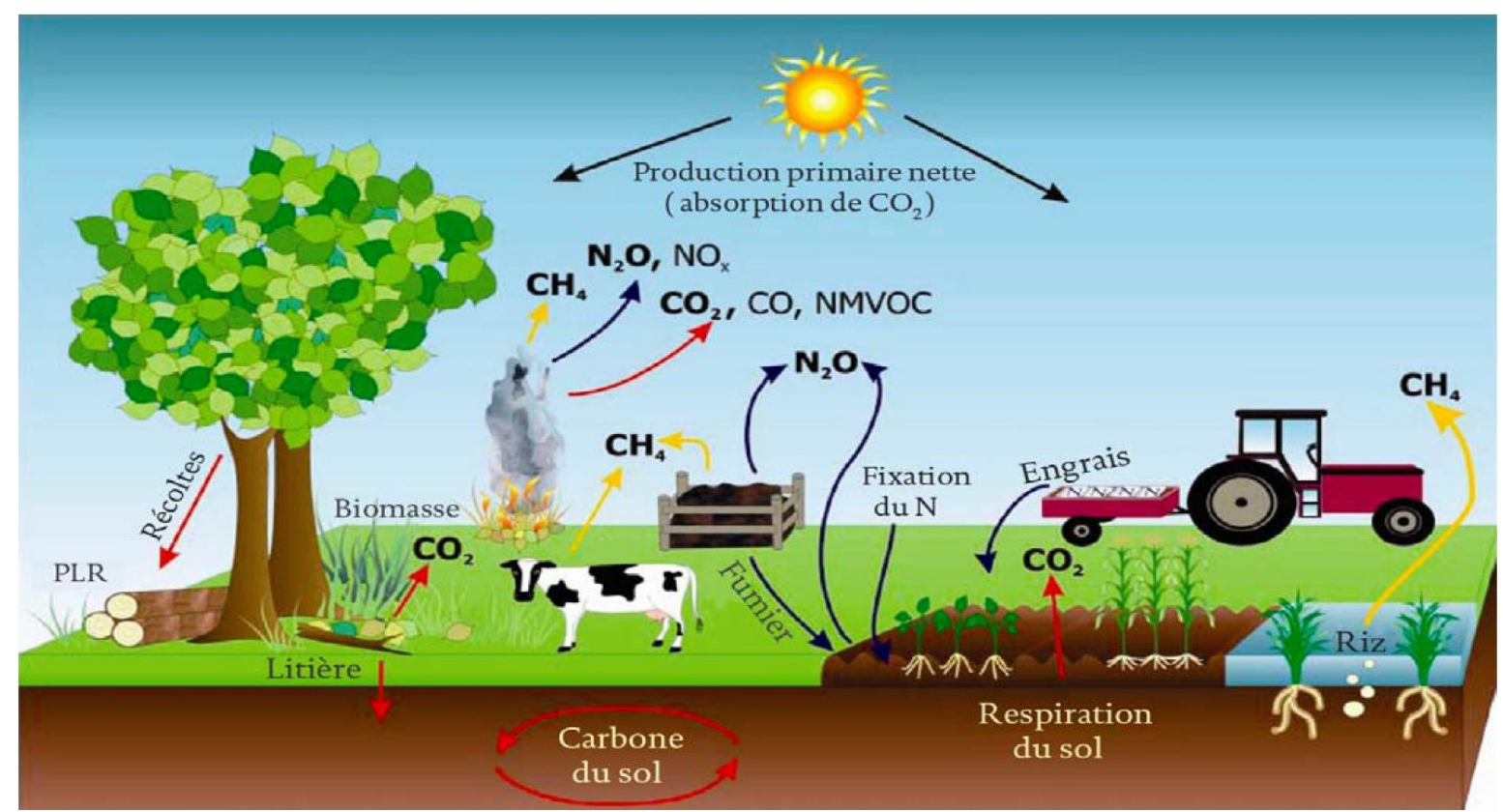

Note : La séquestration du carbone n'est pas représentée explicitement mais joue également un rôle dans le bilan des GES. Source : GIEC (2006) : 16.

De 2000 à 2010, les émissions totales de GES des pays de l'OCDE ont diminué en valeur absolue, au rythme de $0.4 \%$ par an, alors que le volume de la production agricole a progressé de $1.6 \%$ par an (OCDE, 2013 - voir annexe A pour les données par pays) ${ }^{2}$, entraînant une réduction annuelle de l'intensité des émissions $^{3}$ de la production agricole de l'OCDE de $1.97 \%$. Un tel découplage entre la production et les facteurs externes négatifs pour l'environnement est au cœur des objectifs de la croissance verte de l'agriculture (OCDE, 2014). En réduisant leurs émissions tout en réalisant des progrès en terme de productivité et de valeur ajoutée moyennes agricoles, comme le montre le graphique 2, les pays de l'OCDE contribuent au « découplage absolu » défini dans les objectifs d'atténuation (OCDE, 2014) ${ }^{4}$.

Ces résultats s'expliquent essentiellement par l'adoption de techniques et de pratiques de gestion agricole plus efficaces et par la mise en œuvre d'incitations à la réduction des émissions, à la faveur des différentes politiques appliquées par les pays de l'OCDE. Dans plusieurs pays, les politiques publiques ont joué un rôle majeur dans l'adoption de pratiques favorisant l'atténuation des émissions de GES. Ces stratégies prévoient, par exemple, « des conseils aux éleveurs pour améliorer l'efficience de l'alimentation et le rythme de croissance du bétail en vue de limiter les émissions de GES, et des subventions à l'utilisation de biodigesteurs afin de réduire les émissions de $\mathrm{CH}_{4}$ et de remplacer les sources d'énergie grandes émettrices de GES » (OCDE, 2013 : p. 27). L'encadré 1 présente une partie des efforts entrepris en Irlande, à titre d'exemple.

2. Voir OCDE (2013) : p. 57 pour des informations sur les indices de production.

3. L'intensité des émissions correspond aux émissions par unité produite.

4. Le découplage est considéré comme absolu lorsque la variable économique augmente tandis que la variable environnementale est stable ou décroissante (OCDE, 2014). 
Graphique 2. Évolution des émissions de gaz à effet de serre, du produit intérieur brut agricole et de la productivité agricole dans les pays de l'OCDE (1990-2010)

$1990=100$

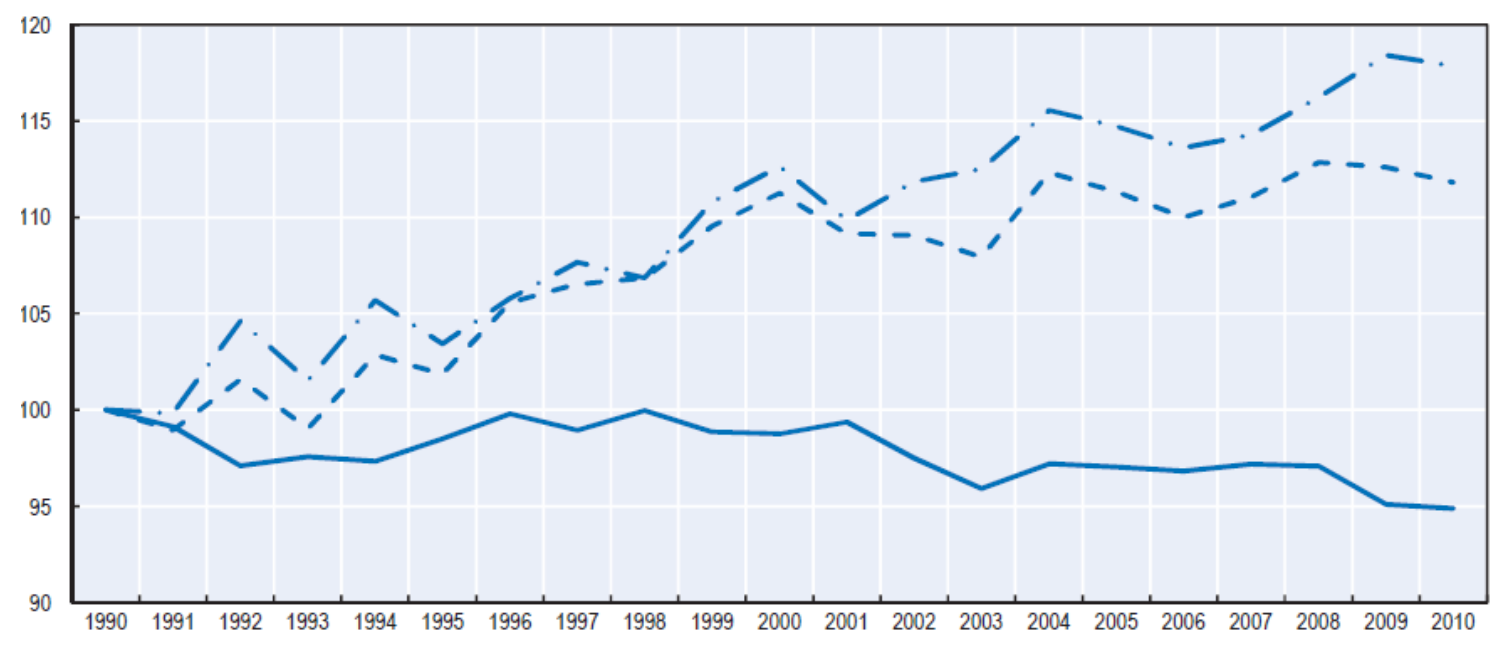

Source : OCDE (2014) à partir des données des inventaires de gaz à effet de serre de la CCNUCC, http://unfccc.int/ghg data/items/3800.php; FAO, FAOSTAT (base de données), http://faostat.fao.org/.

\section{Encadré 1. Atténuation des émissions dans une agriculture fondée sur l'élevage de ruminants - le cas de I'Irlande}

En Irlande, l'agriculture reste la principale responsable des émissions globales de GES, avec $31.1 \%$ des émissions totales nationales qui lui sont imputables (hors consommation énergétique et modification d'affectation des terres). Cette proportion reflète l'importance de l'agriculture dans l'économie irlandaise (elle contribue au total des exportations à hauteur d'environ $11 \%$, soit 10 milliards EUR) et la prépondérance des systèmes d'élevage de ruminants en plein air.

En raison de ces caractéristiques, de nombreux travaux ont été réalisés pour analyser les solutions possibles d'atténuation dans le secteur. Les émissions de l'agriculture irlandaise ont atteint un sommet en 1998, avant d'entamer une baisse constante jusqu'en 2011 sous l'effet de plusieurs mesures, notamment la réforme de la Politique agricole commune, la participation à des initiatives agro-environnementales ou biologiques, le soutien de la gestion du fumier conformément à la Directive sur les nitrates de l'UE, la contribution au boisement et l'exploitation de sources d'énergie renouvelable. Si les émissions d'origine agricole ont légèrement progressé en 2012 et 2013, elles sont restées inférieures de $7.3 \%$ à leur niveau de 1990.

L'empreinte carbone de l'agriculture irlandaise est l'une des plus faibles au monde. En 2011, I'Irlande avait le plus faible taux d'émissions associées à la production de lait de vache (1 kg par kg de produit) et de viande porcine $(4.8 \mathrm{~kg} / \mathrm{kg})$ de l'Union européenne (Leip et al., 2010). Ses émissions par $\mathrm{kg}$ de viande bovine $(18.4 \mathrm{~kg} / \mathrm{kg}$ ) étaient également inférieures à la moyenne de l'UE $(22.2 \mathrm{~kg} / \mathrm{kg})$. En 2013, l'intensité d'émission par calorie de la production alimentaire a diminué d'environ $14 \%$ par rapport à 2005. Des estimations anticipées prévoient que dans un scénario de maintien des conditions actuelles, l'intensité des émissions en 2030 sera inférieure à l'intensité des émissions en 2005 de $25 \%$, contre $35 \%$ en cas d'adoption de nouvelles mesures.

Schulte et Donnellan (2012) ont déterminé un potentiel de réduction de la pollution de $1.1 \mathrm{M} \mathrm{t.} \mathrm{CO}_{2}$ d'ici à 2020, en s'appuyant en grande partie sur une vision de la neutralité carbone proposée par Schulte et al. (2014). Selon leur rapport, l'efficience carbone du secteur agricole peut être améliorée. Des progrès importants ont été réalisés dans l'agriculture et l'agroalimentaire pour réduire les émissions en améliorant les techniques agricoles et la production animale. Le niveau élevé des investissements dans l'innovation, la R-D agricoles et l'agro-alimentaire pendant la période $2005-2012$ a considérablement accéléré la mise au point de nouvelles techniques. II a également permis de faire avancer les vastes travaux consacrés à l'agriculture et la transformation pour trouver des solutions plus intelligentes et respectueuses de l'environnement.

Source : Note de la délégation irlandaise auprès de l'OCDE, à partir de Leip, A. et al. (2010), « Evaluation of the livestock sector's contribution to the EU greenhouse gas emissions (GGELS) », Centre commun de recherche de la Commission européenne, Ispra. ec.europa.eu/agriculture/analysis/external/livestock-gas/full_text_en.pdf ; Schulte, R. et T. Donnellan (2012), « A Marginal Abatement Cost Curve for Irish Agriculture », rapport de la Teagasc, Dublin. www.teagasc.ie/publications/2012/1186/1186_Marginal_Abatement_Cost_Curve_for_Irish_Agriculture.pdf : Schulte, R.P.O. et al. (2013), « Carbon Neutrality as a horizon point for Irish Agriculture : a qualitative appraisal of potential pathways to 2050 », Teagasc, Dublin. www.teagasc.ie/publications/2013/3002/CarbonNeutrality.pdf. 
Pour aller plus loin, il est nécessaire de connaître les différentes solutions envisageables pour atténuer les émissions de GES d'origine agricole et de déterminer si elles sont techniquement efficaces, économiquement efficientes et acceptables pour la société. En particulier, le rapport coût-efficacité des mesures d'atténuation dans l'agriculture, qui a fait l'objet de multiples études, est souvent très variable, parce qu'il dépend de l'emplacement géographique, des conditions climatiques et des pratiques agricoles passées et actuelles; il reste par conséquent difficile à mesurer et à évaluer pour étayer l'action des pouvoirs publics.

Le présent rapport se propose de faire la synthèse et d'analyser les données de plus en plus nombreuses sur le rapport coût-efficacité des mesures d'atténuation des émissions de gaz à effet de serre (GES) dans l'agriculture, à l'appui de la réflexion menée par les responsables publics. Les travaux déjà publiés dans le monde seront passés en revue, les principales tendances et principaux concepts seront expliqués et quelques questions préliminaires et implications pour l'action seront abordées.

Plusieurs réserves non négligeables peuvent néanmoins être émises au sujet de la présente étude. En premier lieu, elle est axée essentiellement sur le rapport coût-efficacité et élude par conséquent d'autres critères tels que les coûts et les avantages connexes ou les coûts de transaction pour les exploitants, essentiellement du fait que ces critères ont rarement été étudiés (voir section 2), alors que les estimations qui en tiendraient compte seraient plus proches de la réalité. En deuxième lieu, l'étude n'est pas une métaanalyse et ne vise pas à évaluer le rapport coût-efficacité global moyen des mesures d'atténuation ou à recommander le recours à des mesures ou des stratégies spécifiques, mais à fournir un aperçu des études publiées et inventoriées ainsi que des estimations qu'elles proposent. En troisième lieu, le présent document a pour thème principal les mesures d'atténuation qui permettent de réduire l'intensité des émissions tout en préservant ou intensifiant la production, afin de pouvoir rechercher simultanément l'atténuation du changement climatique et l'amélioration de la productivité. Ces trois limites sont analysées plus en détail ci-après.

- Le terme «atténuation » peut avoir plusieurs sens. Il désigne souvent une diminution des émissions totales de GES, généralement exprimée sous forme de variation du total des GES émis par une activité spécifique dans une région déterminée et en référence à une année donnée, par exemple : «depuis 1990, les GES émis dans le cadre de l'agriculture et de l'utilisation connexe des sols [en Écosse] ont diminué de 4.2 millions t. $\mathrm{CO}_{2} \mathrm{e} »$ (Gouvernement écossais, 2013a : p. 2). Or, dans le présent rapport, l'atténuation fait référence à une réduction de l'intensité des émissions, à savoir, de la quantité de GES émis par unité produite ou par unité consommée au cours de la production, et s'exprime, par exemple, en $\mathrm{kg} \mathrm{CO}_{2} \mathrm{e} / \mathrm{kg}$ de produit ou $\mathrm{kg} \mathrm{CO}_{2} \mathrm{e} / \mathrm{ha} / \mathrm{année} \mathrm{(voir} \mathrm{MacLeod}$ et al., 2014, pour une synthèse des mesures de réduction de l'intensité GES de la production d'aliments et de boissons) $)^{5}$.

- On distingue les mesures d'atténuation (qui désignent les activités entreprises sur l'exploitation pour réduire les émissions, comme l'ensemencement de cultures de couverture ou l'allègement de l'épandage d'azote) des politiques d'atténuation (qui désignent les instruments d'action des pouvoirs publics pouvant être employés pour encourager l'adoption d'une mesure, par exemple, l'es campagnes d'information ou les incitations).

- Le rapport est centré sur les mesures visant les pratiques productives existantes. La demande alimentaire mondiale devrait augmenter fortement ces trente prochaines années (voir Alexandratos et Bruinsma, 2012). En conséquence, le fléchissement de la production agricole dans les pays de l'OCDE n'engendrera probablement que des hausses compensatoires de la production dans les pays non membres. Étant donné que l'intensité des émissions des pays de l'OCDE est souvent inférieure à celle des autres lors de la production des principales matières premières agricoles (voir Gerber et al., 2013, MacLeod et al., 2013 ; Opio et al., 2013), restreindre cette production dans les pays de l'OCDE

5. Ces deux types d'atténuation induisent une baisse des émissions qui sont moins importantes que si l'on n'avait pas pris de mesures, ce qui n'est pas le cas si la réduction du total des GES est obtenue par un transfert de la production et des sources d'émission de GES. 
reviendrait sans doute à accroître le total net mondial des émissions d'origine agricole, toutes choses égales par ailleurs ${ }^{6}$.

Quatrièmement, la présente étude se consacre aux mesures adoptées du côté de l'offre afin d'atténuer les émissions en réduisant la quantité de GES émis par unité produite ou en renforçant l'absorption et le stockage des GES. Elle n'inclut pas les mesures appliquées du côté de la demande pour atténuer les émissions en modifiant les habitudes et les modes de consommation, en particulier en renforçant l'efficience (par une réduction des déchets alimentaires des ménages, par exemple) ou en substituant certains produits par d'autres à plus faible intensité d'émissions (la viande de bœuf par du poulet, l'acier par du bois ou le gazole minéral par du biogazole, par exemple). Leur absence ne signifie toutefois pas qu'elles n'offrent pas de possibilités. Les mesures applicables du côté de la demande ouvrent d'autres perspectives pour atténuer les émissions de GES mais mériteraient d'être analysées dans une étude distincte, compte tenu de leurs différences fondamentales avec les mesures relatives à l'offre, en termes de stratégies et de quantification de leur rapport coût-efficacité

Enfin, du point de vue de la méthodologie, cette revue se concentre sur le critère de coût-efficacité pour jauger les pratiques de réductions des émissions, mais d'autres méthodes peuvent être employées. McCarl et Schneider (2001) et McCarl et Sands (2007) distinguent des potentiels technique, économique, et compétitif (économique) de réduction des émissions pour différentes stratégies envisagées. Le potentiel technique équivaut au potentiel physique maximum d'une stratégie de réduction des émissions donnée, alors que le potentiel économique prends en compte les coûts (d'opportunité) de cette stratégie. Le potentiel technique définit ainsi la limite supérieure du potentiel économique. Par exemple, Lewandrowski et al. (2004) montrent que le potentiel économique de la séquestration du carbone obtenue en adoptant des pratiques de labour de conservation sur des terres additionnelles, en faisant l'hypothèse d'un prix du carbone maximal, n'atteignait que $25 \%$ du potentiel technique estimé. Le potentiel compétitif (économique) considère qu'une option de réduction va entrer en compétition avec d'autres stratégies de réduction des émissions (labour de conservation, reboisement et biocarburants) et que cela va réduire encore le potentiel économique d'une simple stratégie de réduction des émissions. McCarl et Schneider (2000) démontrent qu'à 100 USD par tonne de carbone, les potentiels technique, économique et compétitif de réduction des émissions sont respectivement de 150, 90, et 65 million de tonnes de carbone équivalent.

La suite du rapport se divise en cinq sections. La section 2 décrit et analyse les méthodes employées pour quantifier le rapport coût-efficacité des mesures d'atténuation. La section 3 passe en revue l'éventail des études récentes sur le rapport coût-efficacité des mesures d'atténuation. La section 4 comporte une série de brèves études de cas pour illustrer certaines difficultés techniques de la quantification du rapport coût-efficacité des mesures d'atténuation. Ces études de cas décrivent également certains obstacles associés à la mise en œuvre des mesures d'atténuation des GES. Les résultats d'un sous-ensemble distinct de mesures d'atténuation ayant déjà fait l'objet de plusieurs études sont comparés à la section 5 afin de montrer les variations possibles du rapport coût-efficacité. Cette section passe également en revue les premières implications pour l'action et est suivie d'une brève conclusion.

\section{Trois grandes méthodes d'évaluation des coûts des mesures d'atténuation : avantages et inconvénients}

\section{Courbes du coût marginal de réduction des émissions}

L'intensification des efforts d'atténuation des émissions de GES agricoles devrait mettre l'accent sur le rapport coût-efficacité (en se concentrant sur des mesures permettant d'atteindre le taux souhaité de réduction à moindre coût) et l'efficience sociale (en réduisant les émissions jusqu'au taux pour lequel les

6. Il est toutefois reconnu que réduire la production peut, dans certains cas (en particulier sur les terres marginales), entraîner une diminution nette des émissions, même lorsque celles de la production déplacée sont prises en compte.

7. Pour approfondir le débat sur l'atténuation par la gestion de la demande, voir Bajželj et al. (2014). 
coûts associés à l'atténuation sont équivalents aux bénéfices de la réduction des émissions pour la société). Diverses études ont tenté de quantifier le rapport coût-efficacité des mesures d'atténuation (sans toutefois tenir compte de l'intégralité des coûts pour la société, la plupart du temps). Cette information est souvent représentée à l'aide d'un outil d'évaluation très répandu pour l'analyse des stratégies, la courbe du coût marginal de réduction des émissions (MACC). Cette courbe affiche le coût de la réduction des émissions par unité supplémentaire (en équivalents $\mathrm{CO}_{2}$ ) et peut être représentée en regard d'une courbe illustrant le bénéfice marginal de réduction des émissions, afin de permettre l'identification du niveau optimal de réduction des émissions (voir graphique 3). Dans les études d'atténuation des émissions de GES, les MACC dérivées de la modélisation sont généralement fluides, alors que celles issues de méthodes d'estimation ascendante des coûts ont plus souvent la forme d'une série de barres discrètes représentant chacune une mesure d'atténuation (voir graphique 4). La largeur de chaque barre représente la réduction de GES et sa hauteur correspond au rapport coût-efficacité de la mesure. La surface couverte par chaque barre équivaut au coût total de la mesure. L'encadré 2 présente un exemple d'application à large échelle de la méthode MACC dans l'optique de comparer les réductions potentielles d'émissions de GES par secteur et région.

\section{Graphique 3. Coûts et avantages marginaux de réduction des émissions}

Pour une mesure technique donnée, la réduction des émissions est optimale lorsque les coûts marginaux de réduction sont égaux aux bénéfices marginaux, c'est-à-dire, à l'intersection des deux courbes

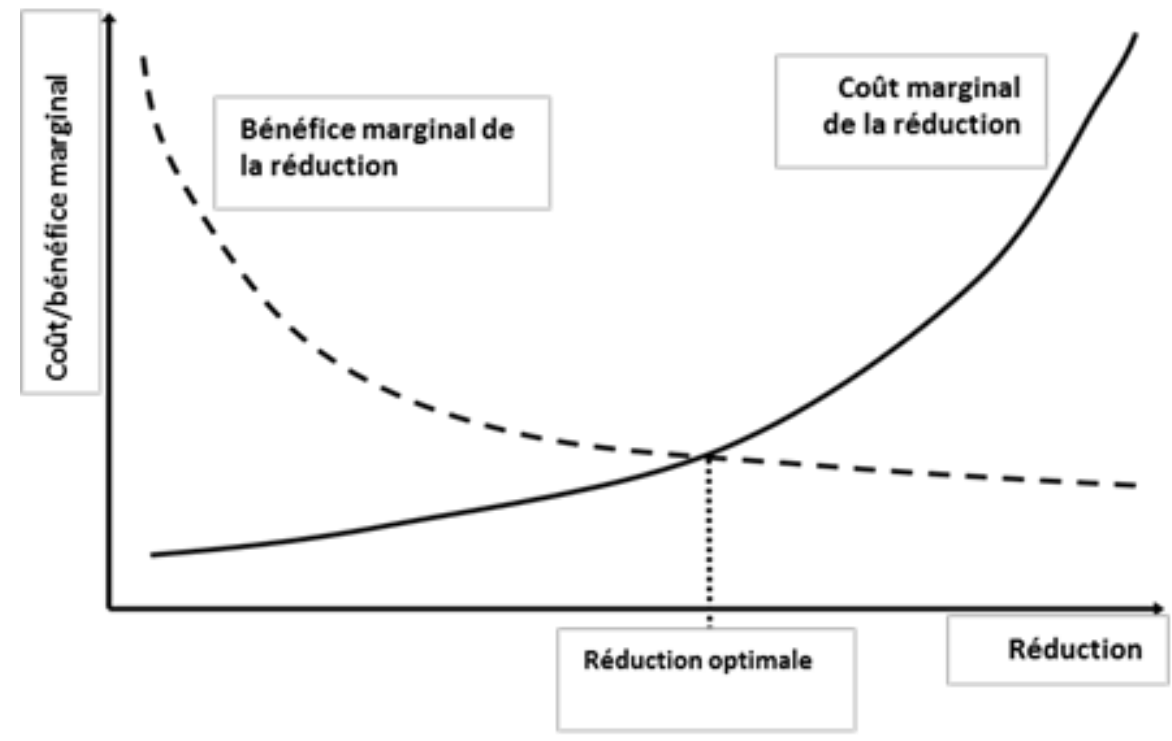

Source : Pearce et Turner (1990). 
Graphique 4. Exemple de courbe du coût marginal de réduction des émissions (MACC) associée aux mesures d'atténuation des émissions de GES du secteur laitier britannique

Coût-efficacité(£/tCO2e)

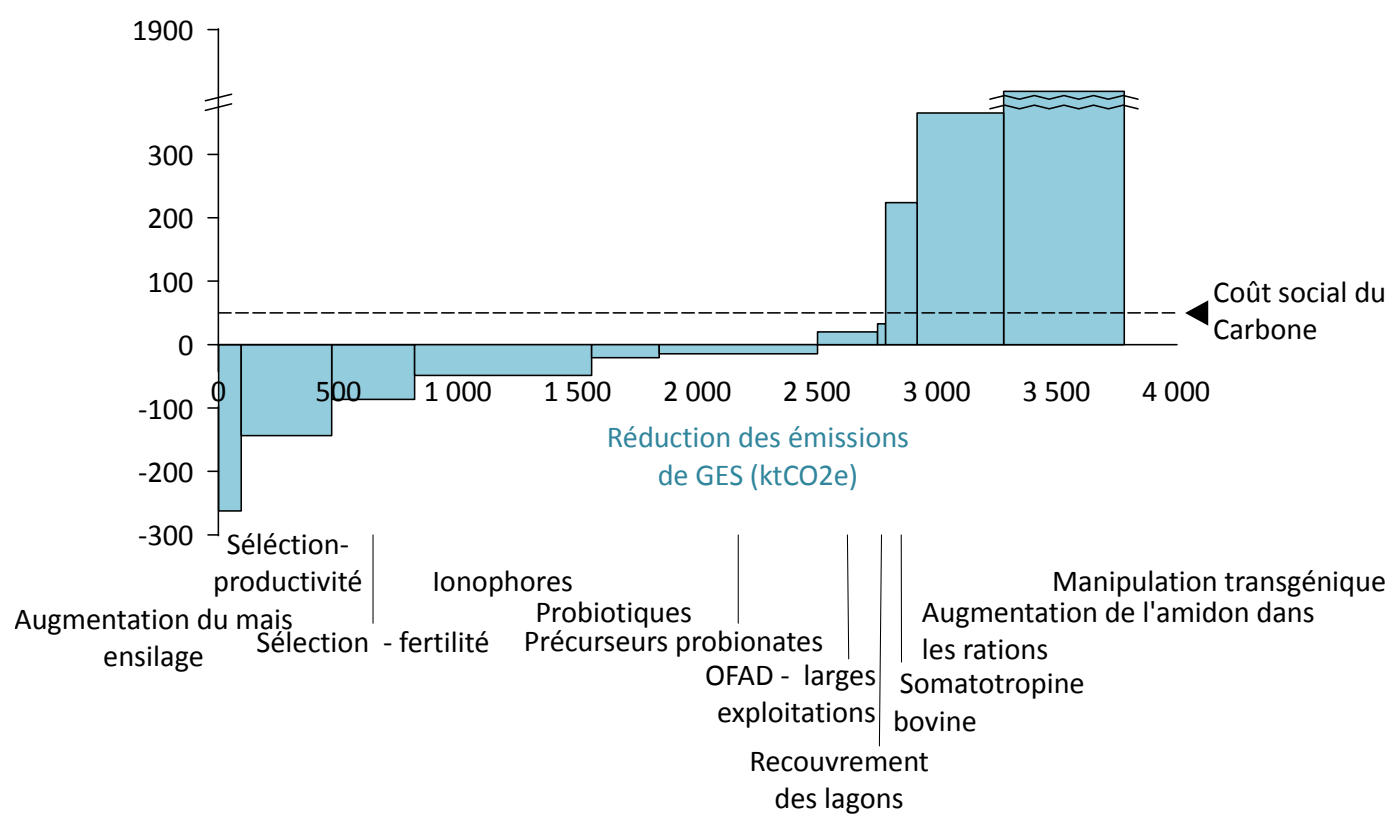

Note : OFAD : digestion anaérobie dans les exploitations agricoles.

\section{Encadré 2. L'utilisation de MACC pour analyser les rapports coût-efficacité projetés des options de réduction des émissions par secteur et pays}

USEPA (2013) a mené une enquête compréhensive des émissions actuelles et projetées des sources de GES non- $\mathrm{CO}_{2}$ $\left(\mathrm{CH}_{4}, \mathrm{~N}_{2} \mathrm{O}\right.$ et autres gaz) et des rapports coût-efficacité de ces mesures au niveau global en 2030. Utilisant une approche ascendante des coûts, le document calcule des MACC comparables pour quatre secteurs dans des pays spécifiques en 2010 et 2030.

\section{Graphique 5. MACC projetée des émissions de GES non- $\mathrm{CO}_{2}$} pour l'agriculture et trois autres secteurs en 2030

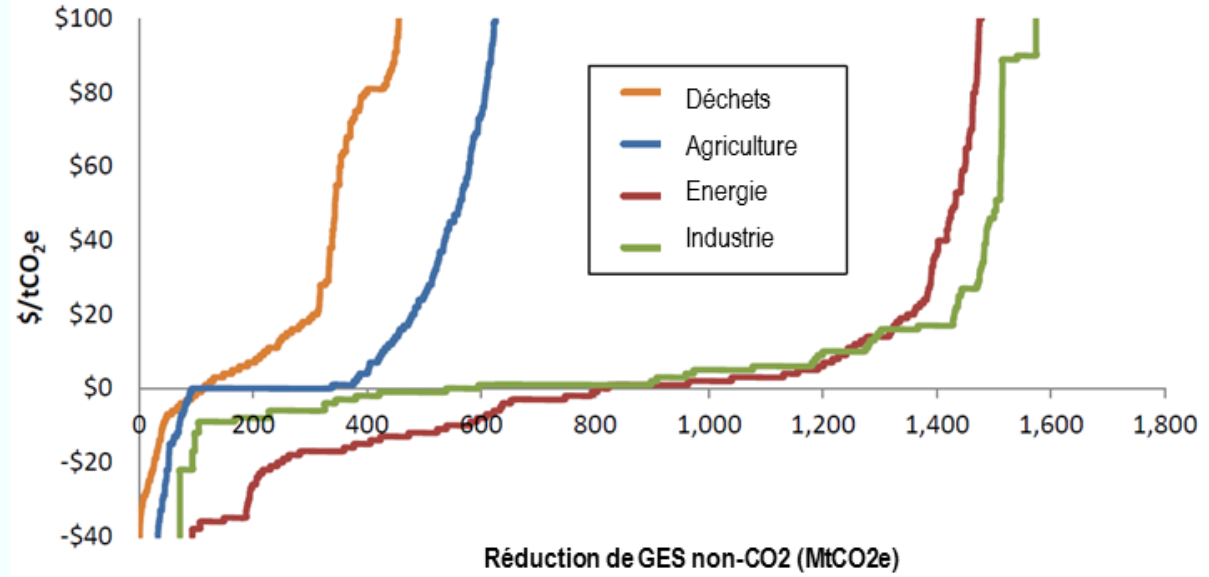

Source : USEPA (2013). 
Encadré 2. L'utilisation de MACC pour analyser les rapports coût-efficacité projetés des options de réduction des émissions par secteur et pays (suite)

Le rapport montre que l'agriculture est et va demeurer la source principale d'émissions de gaz non- $\mathrm{CO}_{2}($ représentant $45 \%$ du total) en 2030. Néanmoins, la réduction des GES agricole n'est pas aussi avantageuse en terme de coût-efficacité que des efforts des secteurs industriels et énergétiques à l'échelle globale, mais est plus avantageuse que des efforts de réduction dans le secteur des déchets (Graphique 5). Par exemple, à un prix de USD $30 / \mathrm{tCO}_{2}$ e, le potentiel de réduction des émissions de l'agriculture est estimé à environ $500 \mathrm{MtCO}_{2} \mathrm{e}$, comparé à $332 \mathrm{MtCO}_{2} \mathrm{e}$ pour les déchets et plus de $1400 \mathrm{MtCO}$ pour les secteurs industriels et énergétiques.

La section agricole du rapport analyse ensuite de manière plus spécifique les trois sources principales des émissions de GES ; l'élevage ( $21 \%$ des émissions projetées de GES non- $\mathrm{CO}_{2}$ en 2030$)$, le riz $(6 \%)$, et les autres terres cultivées (4\%). Dans le cas de l'élevage, l'amélioration de l'efficacité de conversion alimentaire, les suppléments alimentaires, et l'utilisation de digesteur anaérobie sont identifiés comme les principales options à bas coût, mais ne permettent qu'une réduction technique de $10 \%$ des émissions totales en 2030 . L'analyse trouve que $26 \%$ des émissions de $\mathrm{GES} \mathrm{non-CO}_{2}$ provenant de la culture du riz peuvent être réduites via des mesures techniques, et identifie la conversion des terres sèches comme l'option présentant le potentiel le plus important. Enfin, l'absence de labour et la réduction d'application des engrais représentent $80 \%$ du potentiel de réduction des émissions pour les autres cultures (comptant pour $26 \%$ des émissions totales de GES non- $\mathrm{CO}_{2}$ en 2030). Contrairement aux autres cas, la courbe MACC globale pour les terres cultivées se déplace vers la gauche entre 2010 et 2030, démontrant un potentiel de coût-efficacité en diminution progressive, à mesure que le sol devient saturé en carbone.

Source : USEPA (2013), "Global mitigation of non-CO2 Greenhouse gases: 2010-2030", EPA-430-R-13011, USEPA, Washington, DC. http://www.epa.gov/climatechange/EPAactivities/economics/nonco2mitigation.html.

\section{Méthodologies de l'élaboration des MACC}

Le coût marginal de réduction des émissions peut se calculer de différentes manières. Selon Vermont et De Cara (2010), les MACC se répartissent en trois grands types en fonction de la méthode de dérivation des courbes: i) les MACC issues de l'estimation ascendante des coûts; ii) les MACC issues de la modélisation microéconomique, en fonction de prix exogènes ; iii) les modèles d'équilibre de l'offre par région ou par secteur ${ }^{8}$.

\section{i) Estimation ascendante des coûts}

Cette méthode consiste à recueillir des informations sur les coûts ponctuels et récurrents de différentes mesures d'atténuation et sur leurs effets. Ces données servent à calculer a) le coût annuel moyen de chaque mesure ; b) la quantité d'émissions supprimées chaque année ; c) le rapport coût-efficacité, à savoir, le coût par unité d'émissions de GES supprimées. Pour chaque mesure, les résultats sont portés sur un graphique, généralement par ordre croissant de rapport coût-efficacité. Les courbes du coût marginal de réduction des émissions de McKinley (Naucler et Enkvist, 2009) et celles du graphique 4 sont des exemples de ce premier type de MACC.

\section{ii) Modélisation microéconomique, prix exogènes}

S'il est possible de considérer certaines initiatives comme des investissements indépendants, la plupart des mesures du secteur agricole sont adoptées pour être intégrées à des systèmes agricoles soumis à des contraintes spécifiques en termes de terres, de travail et de capital. S'il est estimé que l'exploitation fonctionne à une frontière d'efficience notionnelle, une mesure supplémentaire peut alors déplacer d'autres activités de production et ainsi créer un coût d'opportunité. L'achat d'une cuve à lisier, par exemple, peut empêcher une exploitation laitière de réaliser d'autres investissements en vue d'améliorer le rendement laitier. Par conséquent, le calcul du véritable coût de mise en œuvre d'une mesure devrait comporter une estimation de ce coût d'opportunité, pouvant être dérivée au moyen d'une modélisation économique à l'échelle de l'exploitation. Un exemple de cette approche est l'évaluation spatiale des coûts d'atténuation des émissions de substances autres que le $\mathrm{CO}_{2}$ par le secteur agricole dans l'UE (De Cara et al., 2005).

8. Les trois approches ne sont toutefois pas indépendantes, la deuxième et la troisième pouvant faire appel aux informations fournies par la première. 
iii) Modèles d'équilibre de l'offre par région ou par secteur

La troisième approche fait appel à des modèles d'équilibre, où les prix sont endogènes. Ces modèles décrivent le comportement qu'adopterait l'économie d'une grande région ou l'un de ses secteurs compte tenu des contraintes liées à l'atténuation des émissions de GES. Weyant et al. (2006) identifient trois types de modèles d'équilibre : les modèles d'équilibre multisectoriel, d'équilibre général agrégé et d'équilibre du marché. Les modèles DICE et RICE, par exemple, couvrent tous les grands secteurs de l'économie mondiale (Nordhaus et Boyer, 1999); le modèle ASMGHG représente le secteur de l'agriculture et la sylviculture aux États-Unis (Schneider et al., 2007) et le modèle CAPRI représente l'agriculture européenne (Dominguez et al., 2009). Ces approches désagrégatives ont la «capacité de saisir les mécanismes de propagation intersectorielle ou internationale et les effets retour provoqués par un choc localisé de l'action publique, tel qu'une politique fiscale ou l'imposition d'une norme dans les secteurs agricole et sylvicole », mais elles nécessitent une grande quantité de données (Povellato et al., 2007).

\section{Choix de la méthode et application}

Les trois méthodes ne concernent pas le même type de coûts, et aucune des trois n'englobe tous les coûts de l'atténuation des émissions de GES. Les mesures d'atténuation peuvent engendrer différents types de coûts, comme le montre le graphique 6. Certaines peuvent entraîner des coûts positifs ou négatifs (autrement dit, des bénéfices). Il a été avancé, par exemple, que le fait de réglementer dans le domaine de l'environnement peut soit réduire, soit renforcer la compétitivité (voir MacLeod et al., 2009, p. 20). De même, les mesures d'atténuation peuvent avoir de multiples retombées secondaires sur l'environnement, qui donnent lieu à un coût ou un bénéfice net. Le graphique 6 renseigne sur l'ampleur des différents types de coûts que chaque méthode peut refléter. Cette classification est toutefois quelque peu approximative et ne signifie pas que ces méthodes traduisent exhaustivement les coûts engendrés dans les limites envisagées. Ainsi, alors que les modèles d'équilibre axés sur l'offre sont à même de refléter les modifications des dépenses et des recettes, ils ne sont pas nécessairement les mieux adaptés pour le faire ; en revanche, leur force réside dans l'illustration des retombées sur les aspects économiques du bien-être. Quelle que soit la méthode utilisée, certains coûts (ou bénéfices) seront exclus et il convient de garder ce constat à l'esprit pour interpréter les résultats.

Une fois admises ces limites, le choix de la méthode dépend du but de l'analyse envisagée. En simplifiant, les modèles d'équilibre sont efficaces pour refléter les interactions entre les différentes composantes économiques et sont utiles pour décrire des phénomènes tels que l'incidence d'une stratégie de réduction des émissions agricoles de GES sur l'offre et la demande. La modélisation microéconomique peut donner une idée de la manière dont, par exemple, l'adoption d'une mesure particulière pourrait affecter les performances économiques des exploitations agricoles selon leur type ou leur taille. Les méthodes d'estimation ascendante sont, en général, plus adaptées pour présenter une vue d'ensemble des mesures techniques possibles pour atténuer les émissions, mais rendent rarement compte des coûts d'opportunité. Enfin, une analyse des coûts et avantages sociaux est sans doute plus adaptée qu'une analyse du rapport coût-efficacité si l'on souhaite étudier les coûts des mesures d'atténuation pour la société.

La recherche économétrique semble indiquer que le choix de la méthode peut infléchir les estimations des coûts de mesures données, en particulier pour les émissions ayant un prix peu élevé, même si aucune de ces méthodes ne peut être considérée comme plus exacte que les autres. À l'aide d'une méta-analyse, Vermont et de Cara (2010) démontrent que les coûts d'atténuation se dégageant des modèles d'équilibre sont toujours inférieurs à ceux ressortant des modèles microéconomiques axés sur l'offre. Les estimations dérivées de ces modèles microéconomiques sont également supérieures à celles issues des méthodes de calcul pour les émissions ayant un prix peu élevé. L'encadré 3 présente plus en détail les mérites relatifs de chaque approche, à travers leur incidence sur les résultats (voir également Povellato et al., 2007 et Kesicki et Strachan, 2011). La principale conclusion à tirer de ces résultats est la nécessité d'interpréter les valeurs absolues des estimations avec précaution. Ils incitent également à utiliser la même méthode pour réaliser des comparaisons du rapport coût-efficacité entre les mesures. 


\section{Graphique 6. Classification des coûts engendrés par l'atténuation des émissions de GES}

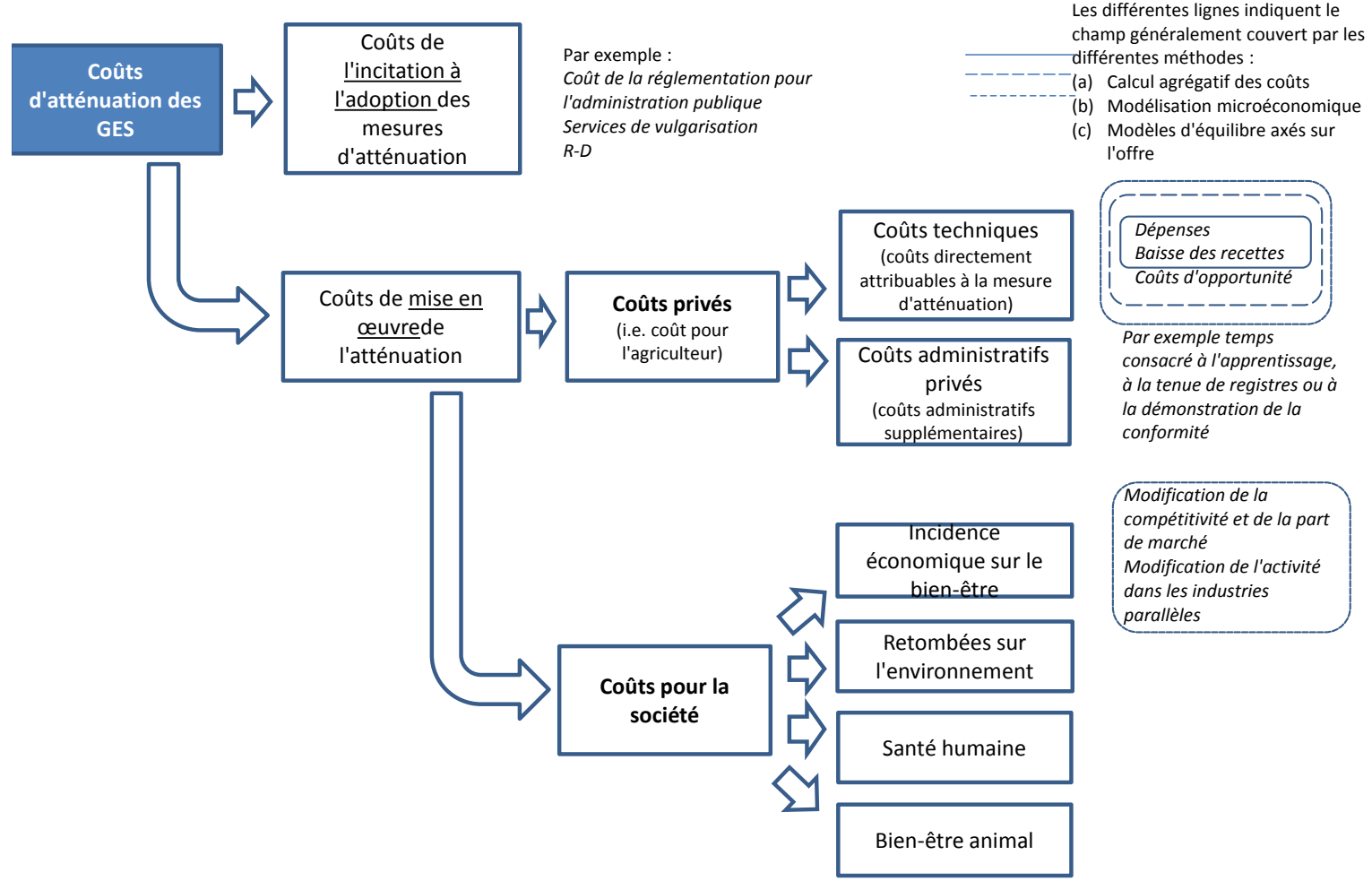

\section{Encadré 3. Implications des différentes approches modélisatrices} résultats d'une méta-analyse

Vermont et Cara (2010) analysent les conséquences du choix de l'approche modélisatrice sur les estimations du potentiel de réduction de la pollution et de ses coûts. Les modèles microéconomiques de l'offre établis en fonction de prix exogènes fournissent une description précise de la batterie d'indicateurs très détaillés relatifs à la viabilité de la production, et les coûts de la réduction de la pollution dérivés de ces modèles coïncident étroitement avec la définition normalisée du coût marginal de réduction de la pollution. Les modèles d'équilibre (partiel ou général) tiennent compte de la demande de produits agricoles et, par conséquent, des prix endogènes, ce qui implique que la stratégie d'atténuation a deux effets : i) un effet direct à travers la modification de l'offre et ii) un effet indirect à travers la modification des prix d'équilibre (qui affecte les coûts d'opportunité de la stratégie d'atténuation). Un taux de flexibilité élevé de l'affectation des ressources dans les modèles d'équilibre peut entraîner des coûts de réduction de la pollution inférieurs à ceux des modèles axés sur l'offre ; néanmoins, les effets de prix du marché peuvent accroître les coûts d'opportunité de l'atténuation et, en conséquence, les estimations du coût de la réduction de la pollution calculées à l'aide des modèles d'équilibre.

Vermont et De Cara (2010) réalisent ensuite une analyse par méta-régression des coûts d'atténuation des émissions de substances autres que le $\mathrm{CO}_{2}$ dans l'agriculture, à partir de 21 études. Leurs résultats démontrent que pour un taux de réduction donné des émissions, les modèles microéconomiques axés sur l'offre aboutissent systématiquement à des coûts de réduction de la pollution supérieurs à ceux issus des modèles d'équilibre (ou, à l'inverse, à un taux de réduction de la pollution inférieur pour un prix donné d'émissions). Par ailleurs, lorsque le prix des émissions est faible (10 EUR/t. $\left.\mathrm{CO}_{2} \mathrm{e}\right)$, les taux de réduction de la pollution des modèles microéconomiques axés sur l'offre sont inférieurs de $60 \%$ à ceux issus des méthodes d'estimation ascendante. Cette différence s'efface à mesure que les prix des émissions augmentent et implique que la majeure partie du potentiel de réduction issu des méthodes d'estimation ascendante s'explique par des pratiques à faibles coûts, voire à coûts négatifs.

Source: Repris de Vermont et De Cara (2010), "How costly is mitigation of non-CO2 greenhouse gas emissions from agriculture?: A meta-analysis », Ecological Economics no 69, pp. 1373-1386. 
Deux variables sont nécessaires au calcul de la largeur des barres d'une MACC — qui représente la réduction ou le potentiel de réduction des émissions de GES sur une période donnée - : le taux de réduction associé à la mesure (la variation de la quantité de GES et/ou du rendement par hectare ou par animal) et la superficie ou le nombre d'animaux supplémentaires auxquels la mesure pourrait s'appliquer sur la période donnée (en plus de la superficie ou du nombre d'animaux actuels).

En théorie, certaines mesures sont applicables à une forte proportion des terres disponibles. Or, la proportion dans laquelle les mesures d'atténuation des émissions de GES sont adoptées en pratique dépend des spécificités de chaque mesure et du cadre politique. Des MACC peuvent être élaborées pour fournir une indication du potentiel de réduction des émissions selon ces différents taux d'adoption. MacLeod et al. (2010), par exemple, distinguent quatre scénarios de réduction possible : réduction technique maximale ; réduction très faisable; réduction moyennement faisable; réduction peu faisable. Le potentiel de réduction technique maximale est la proportion dans laquelle il est possible de réduire les émissions de GES si chaque acteur techniquement capable de mettre en œuvre une mesure le fait autant que possible. Les potentiels de réduction élevé, moyen et faible correspondent au potentiel de réduction dans différents scénarios de stratégies, dont le taux d'adoption a été estimé en fonction d'une analyse de l'adoption et de l'application des stratégies existantes. L'une des difficultés de l'estimation des potentiels de réduction de la pollution consiste à différencier les effets liés à l'adoption d'une mesure du cours normal des choses. De nombreuses pratiques agricoles ne sont pas systématiquement répertoriées, ce qui peut poser des difficultés pour déterminer précisément des mesures de référence (et donc pour mesurer l'évolution).

\section{Limites des MACC}

Si les MACC sont un moyen utile pour restituer les informations, elles comportent certains inconvénients et doivent être interprétées avec précaution. Les principaux inconvénients sont décrits ciaprès (pour plus de détails sur les limites des MACC, voir Eory, 2014 ; Kesicki et Ekins, 2012 ; Vermont et De Cara, 2010).

\section{a) Champ couvert}

Le champ couvert par l'analyse peut être variable et/ou défini de manière imprécise, en termes de limites du système et de catégories de coûts et d'émissions considérées. De ce fait, des possibilités d'atténuation des émissions de GES peuvent être exclues et des estimations de coûts ou de potentiels d'atténuation distordues. Les coûts inclus dépendent en partie de la méthode utilisée (graphique 5). Certains coûts sont plus difficiles à quantifier et sont par conséquent souvent exclus des calculs du rapport coût-efficacité. Il peut s'agir du coût de transactions privées (liées à l'accentuation de l'exposition au risque ou à l'alourdissement de la charge administrative), de coûts pour la société (tels que l'impact secondaire sur l'environnement) ou des coûts engendrés pour faciliter et encourager l'adoption de mesures d'atténuation. Le déplacement des émissions vers des catégories et/ou des lieux hors du champ couvert par l'analyse peut aboutir à une surestimation des potentiels d'atténuation. L'emploi de stratégies fondées sur le cycle de vie peut permettre d'identifier et de quantifier ces effets.

\section{b) Hétérogénéité et incertitudes}

Les MACC sont essentiellement statiques et fournissent en général un bon aperçu des performances moyennes ou normales d'un ensemble de mesures d'atténuation à un instant donné. En réalité, les taux du rapport coût-efficacité et de la réduction des émissions sont susceptibles de varier dans l'espace et dans le temps. Des MACC pourraient être élaborées par types d'exploitations, tailles d'exploitations ou régions représentatifs afin de mieux refléter l'hétérogénéité (voir, par exemple, Dequiedt et Moran 2015).

Les incertitudes liées aux taux d'adoption, aux taux de réduction des émissions et aux coûts sont le résultat de la variabilité naturelle et de notre connaissance limitée des processus biophysiques et sociaux sous-jacents. Si possible, ces incertitudes devraient être quantifiées et incluses dans les MACC. Lorsque 
toute quantification est impossible (dans le cas de politiques encore non appliquées, dont les résultats sont difficiles à prévoir), les incertitudes devraient pour le moins être mentionnées explicitement.

\section{c) Interactions}

Les synergies entre les mesures d'atténuation des émissions (et les incompatibilités éventuelles ainsi que les dépendances au sentier) et leur coût ainsi que leur incidence sur la réduction des émissions sont difficiles à faire apparaître sur des MACC. Les émissions de GES d'origine agricole se caractérisent par des interactions entre différentes sources d'émissions - engrais chimiques et épandage de fumier, affectation des sols à des productions concurrentes ayant chacune une intensité des émissions différente et les approches modélisatrices donnent des résultats différents en fonction du traitement de ces interactions. Les modèles microéconomiques axés sur l'offre tiennent compte des interactions qui existent à l'échelle des exploitations agricoles (contraintes liées à l'alimentation du bétail, par exemple), alors que les modèles d'équilibre reflètent les interactions à travers les effets de prix (modifications du régime alimentaire du bétail et de l'affectation des sols suscitées par l'évolution des prix). Les méthodes de calcul des coûts peuvent permettre de modéliser les interactions, mais de manière moins transparente (Vermont et Cara, 2010). En outre, le potentiel de réduction d'une pratique donnée d'atténuation des émissions de GES n'est pas indépendant de celui des autres pratiques, car elles peuvent exercer entre elles des relations concurrentes ou complémentaires.

En conséquence, une analyse simultanée des pratiques de réduction des émissions de GES est préférable à plusieurs évaluations indépendantes (Schneider et MacCarl, 2006). Pellerin et al. (2013) prennent en compte les interactions entre certaines mesures d'atténuation des émissions de GES et au sein de celles-ci et démontrent que le potentiel cumulé de réduction des émissions diminue de $8 \%$ ou de $18 \%$, en fonction de la méthode de calcul retenue.

\section{d) Taux d'actualisation}

Le choix du taux d'actualisation peut provoquer une distorsion systématique des estimations des coûts, qui favorise ou pénalise les mesures présentant des caractéristiques particulières en matière de coûts et d'atténuation. Si possible, l'élaboration des MACC devrait prendre en compte un ensemble de taux d'actualisation différents afin de permettre la comparaison.

e) Prise en compte des avantages et des coûts secondaires dans l'analyse du rapport coût-efficacité

Les MACC ont pour objectif de présenter le rapport coût-efficacité associé à la réduction d'un type spécifique d'externalité (les émissions de GES, par exemple). Contrairement aux analyses coût-avantages (CBA), elles ont le mérite d'étudier la pollution par unités physiques au lieu de convertir ces unités en unités financières, ce qui supprime un motif d'incertitude supplémentaire lié à la monétisation de l'incidence de la pollution. En revanche, cet accent mis sur une externalité exclut de l'analyse des effets secondaires (la qualité de l'eau ou la biodiversité, par exemple) qui pourraient modifier fortement les résultats (Glenk et Colombo, 2011, Nemet et al., 2010).

Les mesures d'atténuation des GES peuvent avoir divers effets secondaires (voir tableau 26 à l'annexe B), et ne pas les prendre en compte peut devenir un inconvénient pour deux raisons. Premièrement, une MACC ne peut servir à choisir l'affectation des fonds la plus efficace parmi différents objectifs de durabilité (au-delà du changement climatique). La méthode des MACC n'en est pas moins adaptée pour des thèmes de priorité élevée, comme le changement climatique, pour lesquels des seuils de pollution fixés auparavant doivent être atteints, ou lorsque les sources de financement de la réduction d'un polluant particulier ont déjà été choisies. Deuxièmement, si une activité donnée d'atténuation des émissions de GES affecte positivement ou négativement d'autres objectifs de durabilité, notamment si elle limite parallèlement la pollution diffuse de l'eau ou si elle affecte l'adaptation (OCDE, 2104), ces effets secondaires peuvent rendre l'activité d'atténuation des émissions de GES plus souhaitable ou, à l'inverse, moins indiquée que ne le préconiserait une simple mesure du rapport coût-efficacité des GES. 


\section{Encadré 4. Les MACC dans le cas de polluants multiples}

La recherche sur la modélisation des polluants multiples évolue rapidement depuis vingt ans. Deux solutions techniques divergentes existent pour leur intégration. Les polluants peuvent être représentés dans un modèle unique, tel que le modèle GAINS (Amann et al., 2011), qui englobe cinq polluants atmosphériques et six gaz à effet de serre. À l'inverse, l'incidence sur les différents polluants peut être modélisée indépendamment, comme le font Anthony et al. (2008), qui mettent en œuvre six modèles de processus différents pour obtenir des informations sur six polluants. L'approche modélisatrice unique risque de demander un investissement plus important dans la mise au point du modèle mais peut produire des résultats plus cohérents et plus faciles à appliquer à terme, alors que l'autre approche présente l'avantage de donner des résultats plus détaillés et plus solides sur chaque polluant.

L'optimisation comprend également deux grandes approches : l'une consiste à optimiser les émissions d'un polluant tout en présentant les effets des autres (voir par exemple Schneider et al., 2007), alors que l'autre revient à rechercher des solutions optimales qui intègrent les effets de tous les polluants en parallèle (Anthony et al., 2008). Cette intégration peut se faire de trois façons.

- $\quad$ Premièrement, si une unité commune de pollution peut être dérivée des polluants en question, une MACC simple peut être élaborée. Tel est le cas de toutes les MACCS des GES qui prennent en compte plus d'un GES : I'unité commune de mesure est l'équivalent $\mathrm{CO}_{2}\left(\mathrm{CO}_{2} \mathrm{e}\right)$, les gaz autres que le $\mathrm{CO}_{2}$ étant généralement convertis au moyen de valeurs de potentiel de réchauffement planétaire (PRP).

- Deuxièmement, lorsqu'aucune unité physique ne peut être établie aisément pour l'intégration de différents polluants, un indicateur composite peut être mis au point (OCDE, 2008). Pour ce faire, les effets des différents polluants doivent être normalisés pour permettre la comparaison, en confrontant chacun à un objectif propre tel qu'un seuil de nocivité, par exemple, ou à l'aide de techniques de normalisation ou d'établissement d'un plancher et d'un plafond lorsqu'aucun objectif ne peut être fixé. II est également nécessaire de fixer des règles de pondération et d'agrégation. Une analyse multicritères (AMC) peut permettre d'établir une pondération préférentielle des polluants - et d'autres indicateurs, notamment les objectifs et les coûts pour la société - en fonction de l'importance que les pouvoirs publics ou les analystes décident d'accorder à chaque indicateur de l'évaluation (Linkov et al., 2006). Cette méthode permet de prendre en compte des effets sur lesquels les informations sont seulement semi-quantitatives et est appropriée pour faire apparaître les préférences des parties intéressées. Une AMC a été employée dans l'évaluation des leviers d'atténuation des GES (Konidari et Mavrakis 2007) et des stratégies d'adaptation (de Bruin et al. 2009).

- Troisièmement, l'intégration des effets de l'atténuation sur des polluants multiples peut se faire par conversion des unités physiques en valeurs monétaires (Winiwarter et Klimont, 2011), lorsque des estimations du coût des dommages du polluant sont disponibles. La valeur monétaire du dommage évité peut s'ajouter aux coûts financiers de la mesure d'atténuation, pour être ensuite évaluée par rapport à la pollution principale, permettant ainsi d'élargir l'analyse du rapport coût-efficacité aux effets des polluants secondaires. En revanche, si toutes les incidences sur l'environnement sont converties en valeurs monétaires, une analyse coût-bénéfices devient possible (Pretty et al. 2000). Si les résultats de ces approches peuvent être représentés graphiquement, facilitant leur compréhension, le choix des valeurs attribuées aux dommages peut exercer une influence non négligeable sur les résultats. L'utilité de la méthode peut s'en trouver affectée, en particulier lorsque les valeurs des dommages sont très incertaines ou très variables dans le temps ou dans l'espace, ou encore en cas d'effet de seuil important.

Source: Amann, M. et al. (2011), "Cost-effective control of air quality and greenhouse gases in Europe: Modeling and policy applications », Environmental Modelling \& Software no 26, pp. 1489-1501; Anthony, S. et al.(2008), "Quantitative assessment of scenarios for managing trade-off between economics, environment and media ", Rapport № WQ0106 du ministère britannique de l'Environnement, de l'alimentation et des affaires rurales (module 6), ministère britannique de l'Environnement, de l'alimentation et des affaires rurales, ADAS, North Wyke Research ; de Bruin, K., Dellink, R. B. et al. (2009), " Adapting to climate change in the Netherlands: an inventory of climate adaptation options and ranking of alternatives ", Climatic Change no 95, pp. 23-45 ; Konidari, P. et Mavrakis, D. (2007), «A multi-criteria evaluation method for climate change mitigation policy instruments ", Energy Policy no 35, pp. 6235-6257 ; Linkov, I. et al. (2006), "From comparative risk assessment to multicriteria decision analysis and adaptive management: Recent developments and applications ", Environment International no 32, pp. 1072-1093; OCDE (2008), Handbook on Constructing Composite Indicators, Éditions OCDE, Paris ; Pretty, J. N. et al. (2000), "An assessment of the total external costs of UK agriculture », Agricultural Systems no 65, pp. 113-136; Schneider, U. A. et al. (2007), "Agricultural sector analysis on greenhouse gas mitigation in US agriculture and forestry ", Agricultural Systems $n^{\circ}$ 94, pp. 128-140; Winiwarter, W. et Klimont, Z. (2011), « The role of N-gases (N2O, NOx, NH3) in cost-effective strategies to reduce greenhouse gas emissions and air pollution in Europe ", Current Opinion in Environmental Sustainability no 3, pp. 438-445.

Pour surmonter ces obstacles, il est possible de compléter la MACC caractéristique d'un seul polluant par une évaluation qualitative ou quantitative des effets parallèles de sa réduction, ce qui fournirait une ligne d'action à propos de l'incidence globale des mesures d'atténuation. Certaines techniques peuvent également être employées pour faire la synthèse de leurs multiples effets, comme l'illustre l'encadré 4. 
Il importe de tenir compte de ces principales différences et réserves au sujet des méthodes analysées dans la présente section pour comprendre les résultats de l'examen des études publiées et des études de cas de mesures d'atténuation.

\section{3. Étendue des publications analysées : 65 études fournissent une estimation du rapport coût- efficacité de pas moins de 181 mesures d'atténuation des émissions agricoles de GES}

Le rapport a cherché à réunir les résultats de toutes les études récentes (en particulier des dix dernières années), disponibles en anglais, qui évaluent le rapport coût-efficacité de multiples mesures d'atténuation des émissions de GES (les études axées sur une seule mesure n'ont donc pas été prises en compte). Ces études ont été identifiées à l'aide d'une base de données regroupant les travaux consacrés à l'atténuation et mise à jour régulièrement par le Scotland's Rural College (SRUC), à laquelle s'ajoute une bibliographie suggérée par les auteurs du rapport.

Le rapport a identifié 181 mesures d'atténuation distinctes, réunies dans 30 sous-catégories ellesmêmes réparties en huit catégories. Le tableau 1 reprend le nombre de mesures de chaque catégorie et le nombre de fois où les mesures de chaque catégorie ont été incluses dans les études analysées (nombre de reprises), les détails des études étant présentés dans les tableaux 27et 28 de l'annexe. Le tableau 27 comprend uniquement : a) les études du rapport coût-efficacité de mesures d'atténuation spécifiques (et non de catégories générales de mesures ou de stratégies) ; b) les mesures dont le rapport coût-efficacité a été quantifié dans une étude. Par exemple, Schulte et al. (2012) examinent la « santé animale » comme étant une mesure d'atténuation possible, mais sans quantifier son rapport coût-efficacité.

La plupart des études du tableau 1 sont axées sur les mesures d'atténuation des émissions hors $\mathrm{CO}_{2}$ dans les pays de l'OCDE. Alors que certaines études ont été réalisées à l'échelle mondiale, seules quelques-unes se concentrent spécifiquement sur les pays en développement, où les émissions agricoles devraient connaître la plus forte croissance. La plupart des études traitent des mesures d'atténuation des émissions de $\mathrm{N}_{2} \mathrm{O}$ et de $\mathrm{CH}_{4}$ plutôt que de $\mathrm{CO}_{2}$ (issues du changement d'affectation des terres ou de la consommation d'énergie), en raison de leur part et de leur importance respective dans le total des émissions de GES. C'est là un point faible des travaux publiés, étant donné que la consommation d'énergie des exploitations agricoles peut représenter une proportion relativement importante des émissions dans certains sous-secteurs agricoles (viticulture), ou dans les régions où l'eau d'irrigation est pompée dans les nappes souterraines.

Tableau 1. Nombre de mesures d'atténuation de chaque catégorie et nombre total de reprises des mesures d'atténuation de chaque catégorie dans les études analysées

\begin{tabular}{lcc}
\hline \multicolumn{1}{c}{ Catégorie } & Nombre de mesures & Nombre de reprises \\
\hline Gestion des terres labourables & 64 & 179 \\
Gestion des pâturages & 16 & 34 \\
Gestion des sols organiques & 1 & 2 \\
Restauration des terres dégradées & 4 & 5 \\
Gestion du bétail & 49 & 120 \\
Stabulation et fumier & 24 & 67 \\
Changement d'affectation des terres & 7 & 7 \\
Efficacité énergétique & 16 & 23 \\
\hline
\end{tabular}


Bien que les approches ne soient pas toujours présentées clairement et exhaustivement, les méthodes d'estimation ascendante des coûts sont les plus courantes. Outre les inconvénients de cette approche décrits à la section 2 , son utilisation peut aboutir à des mesures de coûts négatives (voir graphique 4, notamment), impossibles à dériver selon des modèles d'équilibre ou axés sur l'offre. Alors que ces coûts négatifs pourraient être considérés comme une illusion modélisatrice, ils peuvent malgré tout aider à évaluer le rapport coût-efficacité global des mesures, en particulier pour classer des mesures dont les résultats des évaluations sont similaires.

En termes de limites du système, la majorité des études se concentre sur les émissions au niveau de l'exploitation ; relativement peu de travaux font appel à une analyse du cycle de vie (ACV) pour quantifier les émissions. ${ }^{9}$ Les actions entreprises au niveau de l'exploitation (modification de l'alimentation du bétail ou des engrais utilisés) peuvent avoir une incidence en amont et en aval de l'exploitation, mais peu d'estimations comparables du rapport coût-efficacité ont été réalisées à ce sujet.

Enfin, les estimations du rapport coût-efficacité reposent sur l'état actuel des connaissances, mais la recherche, technologie et développement (RTD) devraient amener à identifier de nouvelles possibilités d'atténuation et à élargir le potentiel d'atténuation des solutions existantes. ${ }^{10}$ MacLeod et al. (2010c) ont repéré un ensemble de mesures pour lesquelles la RTD devrait permettre de renforcer sensiblement l'atténuation. Ce rapport souligne également la nécessité de comprendre les problèmes socioéconomiques notamment les obstacles techniques à l'adoption des mesures, leur acceptabilité par les consommateurs et la façon de faire apparaître leurs avantages. Plusieurs initiatives misent actuellement sur la RTD pour réduire les émissions de GES. La Global Research Alliance on Agricultural Greenhouse Gases, par exemple, consacre des recherches aux sous-secteurs agricoles de la riziculture, des cultures et de l'élevage, ainsi que à l'étude de thèmes transversaux (cycles du carbone du sol et de l'azote et problèmes de stocks et de mesure $)^{11}$.

\section{4. Études de cas de mesures spécifiques : des coûts très divers}

Pour illustrer avec plus de précision le champ couvert par les mesures d'atténuation, la présente section détaille neuf brèves études de cas consacrées aux éléments suivants :

1. les cultures de couverture ;

2. l'apport raisonné de nutriments ;

3. les inhibiteurs de nitrification ;

4. le changement d'affectation des terres par boisement des pâturages ;

5. la complémentation en lipides dans l'alimentation des ruminants ;

6. la réduction du $\mathrm{CH}_{4}$ issu de la fermentation entérique par amélioration génétique ;

7. la digestion anaérobie ;

8. l'amélioration de l'efficacité énergétique des engins agricoles ;

9. l'exploitation de la fixation biologique de l'azote dans la rotation des cultures et les mélanges herbagers.

9. Pour l'approche ACV, voir par exemple Coderoni et al. (2015), qui emploient l'empreinte carbone pour analyser le potentiel et les coûts associés avec six produits de l'elevage en Italie.

10. Par exemple, la réduction des émissions de $\mathrm{N}_{2} \mathrm{O}$ après l'utilisation de biochar comme amendment du sol a récemment été rapporté dans de nombreuses études. Cayuela et al. (2014) conduisent une métaanalyse des études de laboratoire et de champs (261 expériences) et trouve que l'utilisation de biochar réduit les émissions de $\mathrm{N}_{2} \mathrm{O}$ de $54 \%$.

11. Voir www.globalresearchalliance.org/. 
Les études de cas ont été choisies pour fournir des exemples des principales sources d'émissions de GES dans le secteur agricole des pays de l'OCDE, notamment au niveau de l'exploitation (voir tableau 2). Les études de cas ont également été sélectionnées afin de représenter équitablement les différentes méthodes d'atténuation et afin d'illustrer certains grands enjeux pour l'évaluation du rapport coûtefficacité. Toutefois, certaines sources d'émissions très significatives dans des régions ou pays particuliers mais dont l'importance relative est moindre à l'échelle de l'OCDE, comme la riziculture inondée en Asie de l'Est - source d'émissions de $\mathrm{CH}_{4}$ - ne figurent pas dans l'étude. Il convient également de remarquer que les études de cas portent essentiellement sur des mesures dont le rapport coût-efficacité a été quantifié. D'autres mesures peuvent présenter un potentiel d'atténuation digne d'intérêt mais ne pas encore avoir produit suffisamment d'éléments probants. À titre d'exemple, l'encadré 5 présente deux mesures en cours de développement appliquées essentiellement en France, qui pourraient offrir des possibilités d'atténuation, entre autres avantages. Dans l'ensemble, le choix des études de cas ne signifie en aucune manière que les mesures retenues soient prioritaires par rapport aux autres mesures d'atténuation, mais plutôt qu'elles sont à prendre comme des exemples caractéristiques.

Tableau 2. Principales sources d'émissions au niveau de l'exploitation visées par les mesures d'atténuation des études de cas

\begin{tabular}{|c|c|c|c|c|c|c|c|c|}
\hline Étude de cas & $\begin{array}{l}\mathrm{CH}_{4}: \\
\text { fermen- } \\
\text { tation } \\
\text { entérique }\end{array}$ & $\begin{array}{l}\mathrm{CH}_{4}: \\
\text { gestion } \\
\text { du } \\
\text { fumier }\end{array}$ & $\begin{array}{l}\mathrm{N}_{2} \mathrm{O}: \\
\text { gestion du } \\
\text { fumier }\end{array}$ & $\begin{array}{l}\mathrm{N}_{2} \mathrm{O}: \\
\text { pâturage } \\
\quad \mathrm{s}\end{array}$ & $\begin{array}{l}\mathrm{N}_{2} \mathrm{O}: \\
\text { sols }\end{array}$ & $\begin{array}{c}\mathrm{CH}_{4}: \\
\text { riz }\end{array}$ & $\begin{array}{c}\mathrm{CO}_{2}: \\
\text { carburant }\end{array}$ & $\begin{array}{l}\mathrm{CO}_{2}: \\
\text { stocks } \\
\text { de } \\
\text { carbone }\end{array}$ \\
\hline Cultures de couverture & & & & & $\mathrm{x}$ & & & $x$ \\
\hline Apport raisonné de nutriments & & & & & $x$ & & & \\
\hline Inhibiteurs de nitrification & & & & $\mathrm{x}$ & $\mathrm{x}$ & & & \\
\hline $\begin{array}{l}\text { Changement d'affectation des } \\
\text { terres }\end{array}$ & & & & & & & & $x$ \\
\hline $\begin{array}{l}\text { Complémentation en lipides } \\
\text { dans l'alimentation des } \\
\text { ruminants }\end{array}$ & $x$ & & & & & & & \\
\hline $\begin{array}{l}\text { Réduction du } \mathrm{CH}_{4} \text { issu de la } \\
\text { fermentation entérique par } \\
\text { amélioration génétique }\end{array}$ & $x$ & & & & & & & \\
\hline Digestion anaérobie & & $x$ & & & & & & \\
\hline $\begin{array}{l}\text { Amélioration de l'efficacité } \\
\text { énergétique du matériel }\end{array}$ & & & & & & & $x$ & \\
\hline $\begin{array}{l}\text { Recours à la fixation } \\
\text { biologique de l'azote }\end{array}$ & & & & & $x$ & & $\mathrm{x}$ & \\
\hline
\end{tabular}

Pour chaque étude de cas, sont analysés l'impact la réduction des émissions de GES et son coût, les autres coûts et avantages ainsi que les problèmes rencontrés pour appliquer les mesures requises. On s'attache plus particulièrement aux sources d'émissions plus directement concernées par les politiques de l'OCDE, à savoir, les émissions produites dans la zone de l'OCDE plutôt qu'importées (issues de la production d'aliments pour animaux et d'engrais hors OCDE). Les méthodes de mesure conventionnelles de la CCNUCC sont ainsi respectées, mais il convient de garder à l'esprit l'incidence complexe que ce choix implique sur la filière d'approvisionnement. 


\section{Encadré 5. Exemples de mesures d'atténuation alternatives}

L'accroissement des surfaces de pâturages et la meilleure gestion des pâturages permettent d'augmenter le stockage de carbone dans le sol (Soussana et al., 2007, 2010 ; INRA, 2013). Le potentiel de ce stockage par les prairies est fonction des caractéristiques pédoclimatiques du milieu, mais également des modalités de gestion : affectation des terres (prairies permanentes, ou prairies temporaires entrant en rotation), type de pratiques (durée de vie de la prairie temporaire, nombre de coupes...) ou intensité de l'exploitation (fertilisation et densité de stockage). Des études récentes montrent que les pratiques d'élevage peu intensives augmentent le stockage de carbone (séquestration) tout en diminuant les émissions de GES provenant du sol $\left(\mathrm{N}_{2} \mathrm{O}\right)$ et de l'animal $\left(\mathrm{CH}_{4}\right)$ (Soussana et al., 2010). En effet, une meilleure gestion des pâturages permet : 1) le stockage de carbone dans les sols ; 2) la réduction des émissions de $\mathrm{N}_{2} \mathrm{O}$ liées à la gestion des effluents ; 3) la réduction des émissions de $\mathrm{CO}_{2}$ liées à la consommation d'énergie fossile (carburant) lors des interventions culturales sur les prairies et 4) la réduction des émissions de $\mathrm{CH}_{4}$ liées directement à la conduite de l'élevage et indirectement à la gestion des effluents.

En pratique, cette meilleure gestion des pâturages se traduit par les actions suivantes (INRA, 2013) : allonger la durée de pâturage en moyenne de 20 jours (Arrouays, 2002) ; accroître la durée des prairies temporaires ; désintensifier la fertilisation des prairies permanentes et temporaires ; intensifier la rentabilité des prairies les moins productives par augmentation du chargement. Si la réduction des émissions liée à l'augmentation des surfaces de pâturages et l'amélioration de leur gestion est relativement facile à mesurer, il est encore à ce stade délicat d'estimer la réduction des émissions indirectes qui peut y être associée. Des effets indirects en amont et en aval de l'exploitation sont en effet attendus. En amont, des effets indirects positifs sir les émissions de GES sont attendus en lien avec la réduction des achats d'aliments pour les animaux (fabrication et transport) et en lien avec d'éventuels changements d'utilisation des terres (déforestation) qui auraient pu prendre place pour produire les tourteaux de soja. D'autres effets induits en amont sont associés aux émissions de GES liées à la fabrication et au transport des fertilisants et au carburant utilisé. En aval, les effets positifs induits concernent la réduction des émissions de $\mathrm{N}_{2} \mathrm{O}$ liées aux variations de nitrate potentiellement lessivable et aux émissions de $\mathrm{NH}_{4}$. Ces deux sources indirectes d'émissions de GES sont modifiées par l'évolution des pratiques de fertilisation et de gestion des effluents dans les prairies.

Le développement d'éléments de paysage et d'infrastructures agroécologiques, notamment les haies et l'agroforesterie, permettent de capter le $\mathrm{CO}_{2}$ atmosphérique à travers une augmentation du stockage de carbone dans la matière organique des sols et la biomasse pérenne des arbres (INRA, 2013 ; Arrouays et al. 2002 ; Kumar et Nair, 2011). En plus de la captation de $\mathrm{CO}_{2}$, les systèmes associant les arbres aux cultures annuelles sont aussi susceptibles d'améliorer les revenus des agriculteurs. Si les rendements moyens des cultures peuvent parfois diminuer, la valorisation des bois d'œuvre produits, ainsi que la capacité d'atténuation des pertes de récoltes les années de sécheresse, grâce au fort pouvoir d'adaptation que ces systèmes confèrent à leur milieu (meilleure régulation de l'eau, meilleure captation des nutriments, protection physique - Verchot et al., 2007 -) peuvent améliorer globalement les revenus des agriculteurs (Albrecht et Kandji, 2003).

Source: Albrecht, A. et S.T. Kandji (2003), "Carbon sequestration in tropical agroforestry systems ", Agriculture Ecosystems and Environment 99:15-27 ; Arrouays D. et al. (2002), "Contribution à la lutte contre l'effet de serre. Stocker du carbone dans les sols agricoles de France ?», Expertise scientifique collective, INRA, France ; INRA (2013), "Quelle contribution de l'agriculture française à la réduction des émissions de gaz à effet de serre ?», Expertise collective, INRA, France. http://institut.inra.fr/Missions/Eclairer-les-decisions/Etudes/Toutes-les-actualites/Etude-Reduction-des-GES-enagriculture ; Kumar, B.M. et Nair, P.K.R. (2011), Sequestration potential of agroforestry systems: opportunities and challenges. Advances in Agroforestry, no $8 . \quad$ Springer Science Dordrecht: (http://library.uniteddiversity.coop/Permaculture/Agroforestry/Carbon Sequestration Potential of Agroforestry Systems-

Opportunities and Challenges.pdf Soussana, J.F. et al. (2007), "Full accounting of the greenhouse gas (CO2, N2O, $\mathrm{CH} 4$ ) budget of nine European grassland sites ", Agriculture, Ecosystems and Environment, 121, 121-134. Soussana, J.F. et al. (2010), "Mitigating the greenhouse gas balance of ruminant production systems through carbon sequestration in grasslands ", Animal, 4, 334-350 ; Verchot, L.V. et al. (2007), "Climate change: linking adaptation and mitigation through agroforestry », Mitigation and Adaptation Strategies for Global Change 12 (5) : 901-918.

\subsection{Les cultures de couverture ${ }^{12}$}

\section{Introduction}

Une culture de couverture est une culture à croissance rapide qui pousse en même temps qu'une culture principale ou entre deux de ses récoltes successives. Elle apporte différents avantages, notamment : l'atténuation de l'érosion du sol, l'amélioration de la structure du sol et de la rétention de l'humidité, la fixation de l'azote, la suppression des mauvaises herbes et la constitution d'un biotope favorable aux insectes (Lu et al., 2000). Parmi les cultures de couverture, les cultures dérobées ont pour objectif de récupérer l'excédent d'azote non utilisé après la récolte de la culture principale et, ce faisant, de

12. Cette étude de cas s'appuie en partie sur les travaux de Frelih-Larsen et al. (2014). 
réduire le taux de déperdition de l'azote initialement contenu dans le sol. Une autre culture de couverture possible consiste à planter un couvert végétal temporaire ou permanent dans les vergers et les vignobles.

Les cultures de couverture peuvent atténuer les émissions de GES des quatre manières suivantes : en enrichissant la teneur en carbone des sols organiques; en limitant les pertes de carbone du sol dues à l'érosion pendant la jachère ; en réduisant le lessivage de l'azote (et les émissions de $\mathrm{N}_{2} \mathrm{O}$ ) ; en restreignant l'épandage d'azote nécessaire sur le suivant cultural (et l'utilisation d'engrais de synthèse).

\section{Impact attendu sur les émissions de GES}

La principale incidence des cultures de couverture sur l'atténuation des émissions au niveau de l'exploitation est l'enrichissement du stockage du carbone dans le sol (voir tableau 3). La réduction des émissions de $\mathrm{N}_{2} \mathrm{O}$ par l'absorption du nitrate et de l'ammonium peut être incertaine. Pour cette raison, les auteurs de certaines études (Pellerin et al., 2013 : p. 44) l'ont exclue des calculs de l'atténuation des émissions (MACC pour la France). De faibles réductions des émissions de $\mathrm{CO}_{2}$ peuvent également se produire si les cultures de couverture permettent de réduire la quantité d'engrais de synthèse appliqués, mais elles sont susceptibles d'être neutralisées par la légère hausse de la consommation du gazole entrant en jeu dans la culture de la variété de couverture, comme le montre le tableau 3.

Tableau 3. Réduction des émissions associée aux cultures de couverture semées pendant la période de jachère de la rotation des grandes cultures

\begin{tabular}{|c|c|c|c|}
\hline Forme d'atténuation & $\begin{array}{l}\text { Taux de réduction } \\
\text { des émissions }\end{array}$ & Source & Lieu \\
\hline \multirow[t]{3}{*}{ Enrichissement du sol en C } & $0.874+/-0.393$ t. $\mathrm{CO}_{2} \mathrm{e} / \mathrm{ha} / \mathrm{an}$ & $\begin{array}{l}\text { Pellerin et al. (2013) (à } \\
\text { partir de Justes et al., } \\
\text { 2012) }\end{array}$ & France \\
\hline & $\begin{array}{l}1 \mathrm{t} . \mathrm{CO}_{2} \mathrm{e} / \mathrm{ha} / \mathrm{an} \\
\text { "Hausse légère mais } \\
\text { significative du COS » (carbone } \\
\text { organique du sol) }\end{array}$ & $\begin{array}{l}\text { Schulte et al. (2012) } \\
\text { Kirk et al. (2012) }\end{array}$ & $\begin{array}{l}\text { Irlande } \\
\text { Royaume-Uni }\end{array}$ \\
\hline & 1.75 t. $\mathrm{CO}_{2} \mathrm{e} / \mathrm{ha} / \mathrm{an}$ & $\begin{array}{l}\text { Posthumus et al. } \\
\text { (2013) }\end{array}$ & Royaume-Uni \\
\hline $\begin{array}{l}\text { Réduction des facteurs d'émissions } \\
\text { directes et indirectes de } \mathrm{N}>\mathrm{N}_{2} \mathrm{O}\end{array}$ & $\begin{array}{l}\text { Très variable } \\
0.49 \text { t. } \mathrm{CO}_{2} \mathrm{e} / \mathrm{ha} / \mathrm{an} \\
\text { Réduction du lessivage de } \\
\text { l'azote à hauteur de } 30 \mathrm{~kg} \mathrm{~N} / \mathrm{ha}= \\
0.11 \mathrm{t} . \mathrm{CO}_{2} \mathrm{e} / \mathrm{ha}\end{array}$ & $\begin{array}{l}\text { Pellerin et al. (2013) } \\
\text { Schulte et al. (2012) } \\
\text { Cameron et al. (2002) } \\
\text { (cité dans O'Hara, } \\
\text { 2003) }\end{array}$ & $\begin{array}{l}\text { France } \\
\text { Irlande } \\
\text { Nouvelle-Zélande }\end{array}$ \\
\hline $\begin{array}{l}\text { Réduction de la quantité d'azote } \\
\text { appliqué }\end{array}$ & 0.06 t. $\mathrm{CO}_{2} \mathrm{e} / \mathrm{ha} / \mathrm{an}$ & Pellerin et al. (2013) & France \\
\hline $\begin{array}{l}\text { Intensification de la consommation } \\
\text { de gazole et des émissions connexes } \\
\text { de } \mathrm{CO}_{2} \text { dans le cadre des } \\
\text { interventions culturales }\end{array}$ & -0.062 t. $\mathrm{CO}_{2} \mathrm{e} / \mathrm{ha} / \mathrm{an}$ & Pellerin et al. (2013) & France \\
\hline
\end{tabular}

\section{Éléments des coûts et rapport coût-efficacité}

Des coûts ponctuels peuvent apparaître si l'organisation de la culture de couverture nécessite d'investir dans des semoirs spécialisés ou de réaliser des aménagements plus importants pour se doter d'autre matériel (tel que semoirs de précision ou charrues) afin de gérer des quantités accrues de résidus. Qui plus est, les agriculteurs doivent parfois investir dans le matériel approprié afin de «brûler» la culture de couverture ou de mettre un terme à sa croissance avant l'ensemencement de la culture principale. L'achat de semences et les interventions culturales supplémentaires pour la culture, la destruction et l'incorporation de la variété de couverture sont sources de coûts récurrents. La diminution des taux d'épandage d'engrais de synthèse peut permettre de réaliser des économies (voir tableau 4).

Globalement, les cultures de couverture sont classées comme susceptibles d'entraîner des coûts significatifs. Le principal motif de variation du rapport coût-efficacité est probablement le coût de la culture et de l'incorporation de la variété de couverture, qui dépend de l'efficience de la culture. Cette 
opération n'aurait sans doute pas un bon rapport coût-efficacité dans les zones où les coûts associés à la culture sont élevés, ou lorsque l'utilisation de cultures de couverture risque de réduire le rendement.

Tableau 4. Principaux coûts des cultures de couverture (les données entre parenthèses signalent des économies).

\begin{tabular}{|c|c|c|c|}
\hline Coûts/économies récurrents & $\begin{array}{l}\text { Coût total ou } \\
\text { économie totale }\end{array}$ & Étude & Lieu \\
\hline Diminution des achats d'engrais & (41 EUR/ha/an) & Pellerin et al. (2013) & France \\
\hline $\begin{array}{l}\text { Ensemencement et destruction de la culture de } \\
\text { couverture }\end{array}$ & $160 \mathrm{EUR} / \mathrm{t} . \mathrm{CO}_{2} \mathrm{e}$ & & \\
\hline $\begin{array}{l}\text { Achat de semences et coûts de carburant liés à la } \\
\text { culture de la variété de couverture }\end{array}$ & $\begin{array}{l}71 \mathrm{EUR} / \mathrm{ha} / \mathrm{an} \\
\sim 50 \mathrm{EUR} / \mathrm{t} . \mathrm{CO}_{2} \mathrm{e}\end{array}$ & Schulte et al. (2012) & Irlande \\
\hline $\begin{array}{l}\text { Semences (70 EUR/ha/an) } \\
\text { Culture/Semis ( } 76 \mathrm{EUR} / \mathrm{ha} / \mathrm{an}) \\
\text { Incorporation des résidus de culture } \\
\text { (32 EUR/ha/an) }\end{array}$ & $178 \mathrm{EUR} / \mathrm{ha} / \mathrm{an}$ & $\begin{array}{l}\text { Posthumus et al. } \\
\text { (2013) }\end{array}$ & Royaume-Uni \\
\hline
\end{tabular}

Source : références citées.

\section{Autres coûts et avantages}

Les cultures de couverture sont réputées avoir de multiples autres avantages. En particulier, elles peuvent réduire les émissions de GES issues de la fabrication d'engrais si elles permettent de diminuer l'épandage d'engrais de synthèse (Pellerin et al., 2013 : p. 45). L'adaptation des cultures de couverture peut également présenter des avantages, comme la baisse de l'érosion des sols et l'accroissement de leur capacité de rétention de l'eau. Le ralentissement du ruissellement peut également améliorer la qualité de l'eau (Schulte et al., 2012 : p. 39 ; Kirk et al., 2012 : p. 36 ; Wiltshire et al., 2014 : p. 23).

En revanche, elles peuvent provoquer des pertes de production si elles contraignent à passer de cultures d'hiver à des cultures de printemps (Wiltshire et al., 2014: p. 24). Une corrélation existe également avec une utilisation plus intense des herbicides (Schulte et al., 2012 ; Wiltshire et al., 2014 : 2014 : p. 23).

\section{Difficultés de mise en ouvre}

Certaines stratégies ont encouragé le recours aux cultures de couverture. Le programme Agriculture et développement rural de l'UE a servi à verser des paiements à l'hectare pour l'ensemencement de cultures de couverture sur les terres labourables en rotation culturale pendant la période de jachère. Cependant, des difficultés existent pour définir la référence et estimer les taux probables d'application supplémentaire.

Les cultures de couverture doivent être soigneusement ciblées pour que l'atténuation des émissions de GES soit efficace par rapport à son coût. Hristov et al. (2013 : p. 100) soulignent que « [1]es interactions avec les autres pratiques de conservation du sol sont indéniables (lors de la préparation du sol, par exemple) et doivent être prises en compte lorsque la culture de couverture a pour but de réduire les émissions de GES de toute l'exploitation. » Alors que Regina et al. (2013) démontrent qu'en milieu boréal, le sol nu peut émettre directement un taux élevé de $\mathrm{N}_{2} \mathrm{O}$, « [l]es données sur l'incidence de pratiques telles que les cultures de couverture, les cultures dérobées, l'application fractionnée d'engrais ou l'agriculture de précision sont toujours insuffisantes ».

L'adoption des cultures de couverture peut être freinée par différentes considérations : leur mise en place coïncide avec une période déjà chargée dans le calendrier agricole (Kirk et al., 2012 : p. 34) ; elles raccourcissent la durée de culture du suivant cultural (Wiltshire et al., $2014:$ p. 21) ; elles augmentent le coût des semences et de la culture (Kirk et al. 2012: p. 34 ; Wiltshire et al., 2014: 21) ; elles sont incompatibles avec d'autres pratiques agricoles, telles que le pacage après récolte (Wallander, 2013). La mise en place ou la destruction de la culture de couverture en milieu humide peut risquer d'endommager le sol (Kirk et al., 2012 : p. 34), et les cultures de couverture peuvent avoir une incidence négative sur le rendement du suivant cultural (Wiltshire et al., 2014 : p. 21). Des préoccupations ont également été 
soulevées au sujet du recours et de la résistance aux herbicides, ainsi que du manque de terres adaptées (Wiltshire et al., 2014 : p. 21). Par exemple, les cultures de couverture conviennent mieux aux sols légers en raison de la nécessité de labourer au printemps, et aux sols perméables à texture légère afin de permettre la préparation d'un sol de bonne qualité pour le suivant cultural. De même, elles sont sans doute plus indiquées pour les régions où les précipitations printanières sont relativement élevées, étant donné que la culture de couverture peut appauvrir les réserves hydriques du sol.

\subsection{L'apport raisonné de nutriments ${ }^{13}$}

\section{Introduction}

L'agriculture de précision englobe de nombreuses technologies qui apportent des informations plus précises sur les terres et les cultures, permettant ainsi à l'agriculteur de réagir aux variations sur le terrain en prenant des décisions relatives à l'épandage d'engrais ou de produits phytosanitaires, à l'irrigation, à l'ensemencement, etc., de manière différenciée, au lieu de réaliser des travaux agricoles non ciblés. Jusqu'à présent, l'accent a été mis sur le perfectionnement de la production végétale, mais les technologies de l'agriculture de précision peuvent également s'appliquer aux pâturages.

L'agriculture de précision peut offrir des avantages dans les champs où le rendement varie en fonction d'un schéma prévisible lié aux différences en termes de qualité des sols, d'envahissement des mauvaises herbes ou de drainage. Elle donne également la possibilité de limiter le chevauchement physique entre les passages des machines. L'agriculture de précision se décompose en cinq grands groupes de technologies (Rains et Thomas, 2009).

- Les systèmes d'information géographique (GIS) sont des logiciels des gestion des données spatiales.

- Les systèmes de localisation GPS différentiels (DGPS) fournissent des informations topographiques sur les positions employée par les GIS avec la précision nécessaire sur le terrain.

- Les capteurs procèdent à des mesures relatives aux propriétés du sol, aux ravageurs, à l'état sanitaire des cultures, etc. Ils peuvent être installés sur les tracteurs ou fonctionner à distance, notamment par photographie aérienne ou satellitaire.

- Le contrôle fonctionnel de rendement est la mesure du rendement des cultures pendant la récolte, qui permet de dresser une carte du rendement.

- La technologie des taux variables est constituée de régulateurs informatiques et du matériel connexe afin de moduler l'introduction d'engrais, de chaux, de semences, de pesticides, etc. sur les différentes zones d'un champ donné.

Plus est élevé le degré de précision spatiale, généralement compris entre $\pm 40 \mathrm{~cm}$ et $\pm 2 \mathrm{~cm}$, plus les investissements requis sont importants. Les équipements les plus élémentaires permettent de réduire le nombre de passages des machines grâce à des dispositifs d'aide visuelle au conducteur (technologies GPS et GIS), alors que l'utilisation de la VRT nécessite un investissement supérieur pour que les machines soient équipées de la conduite automatique ${ }^{14}$.

\section{Impact attendu sur les émissions de GES}

Le principal impact de cette technologie sur les émissions de GES est la baisse de l'utilisation d'engrais azotés et la réduction consécutive des émissions de $\mathrm{N}_{2} \mathrm{O}$ provenant des sols. Ces effets n'ont pas encore été bien étudiés, mais le tableau 5 présente quelques estimations.

13. Cette étude de cas s'appuie en partie sur les travaux d'Eory (2012).

14. Communication personnelle avec R.M. Rees (SRUC). 
Tableau 5. Taux de réduction des émissions pour l'apport raisonné de nutrients

\begin{tabular}{lccl}
\hline \multicolumn{1}{c}{ Forme d'atténuation } & Réduction \% & Référence & Lieu \\
\hline $\mathrm{N}_{2} \mathrm{O}$ du sol dû aux engrais & $5 \%$ & (Bates et al., 2009) & UE-27 \\
$\mathrm{N}_{2} \mathrm{O}$ du sol dû aux engrais & $15 \%$ & $(\mathrm{ICF}, 2013$ et al.) & États-Unis \\
$\mathrm{N}_{2} \mathrm{O}$ du sol dû aux engrais & $34 \%$ & $(\mathrm{ICF}, 2013$ et al.) & États-Unis \\
$\mathrm{N}_{2} \mathrm{O}$ du sol dû aux engrais & 0.2 t. $\mathrm{CO}_{2} \mathrm{e} / \mathrm{ha}$ & (Moran et al., 2008) & Royaume-Uni \\
\hline
\end{tabular}

Source : références citées.

\section{Éléments des coûts et rapport coût-efficacité}

Les avantages financiers de l'agriculture de précision comprennent la diminution de l'utilisation d'engrais et de produits agrochimiques, la baisse de la consommation de carburant, l'amélioration du rendement et le renforcement de la qualité des cultures (ICF, 2013). On estime que ces bénéfices peuvent représenter jusqu'à $80 \mathrm{EUR} \mathrm{ha}^{-1}$ (Bates et al., 2009 ; Boyer et al., 2011 ; Godwin et al., 2003 ; HGCA, 2009).

Les coûts ponctuels liés à l'achat d'équipement (matériel et logiciels) et à la formation sont compris entre 6000 et 50000 EUR (HGCA, 2009; ICF, 2013), auxquels s'ajoutent les frais annuels de fonctionnement et d'entretien et ceux de suivi des sols et des cultures.

Les rapports coût-efficacité estimés présentent une grande diversité (tableau 6). Ils dépendent en grande partie du coût de l'équipement, de la taille de l'exploitation et de l'hypothèse de réduction des GES retenue. Par exemple, le rapport coût-efficacité de la technologie mise en œuvre aux États-Unis (technologie GreenSeeker ${ }^{\mathrm{TM}}$, qui détecte l'état des cultures pour ajuster au plus près les taux d'épandage de l'azote) a été estimé entre <0EUR et $332 \mathrm{EUR} / \mathrm{t} . \mathrm{CO}_{2} \mathrm{e}$, en fonction de son impact supposé sur la réduction des émissions de $\mathrm{N}_{2} \mathrm{O}$ (estimée à $15 \%$ ou $34 \%$ selon le scénario retenu, soit en moyenne 29 EUR/t. $\mathrm{CO}_{2} \mathrm{e}$ pour le scénario d'une faible réduction des émissions et $66 \mathrm{EUR} / \mathrm{t} . \mathrm{CO}_{2} \mathrm{e}$ pour le scénario d'une forte réduction), de la taille de l'exploitation (en moyenne $7 \mathrm{EUR} / \mathrm{t} . \mathrm{CO}_{2} \mathrm{e}$ pour une ferme de 400 ha et $108 \mathrm{EUR} / \mathrm{t} . \mathrm{CO}_{2} \mathrm{e}$ pour une ferme de $100 \mathrm{ha}$ ), du type de céréales (en moyenne $13 \mathrm{EUR} / \mathrm{t} . \mathrm{CO}_{2} \mathrm{e}$ pour le maïs et $82 \mathrm{EUR} / \mathrm{t}$. $\mathrm{CO}_{2}$ e pour le blé) et de la région (en moyenne $21 \mathrm{EUR} / \mathrm{t} . \mathrm{CO}_{2}$ e pour la « Corn Belt » et $62 \mathrm{EUR} / \mathrm{t}$. $\mathrm{CO}_{2} \mathrm{e}$ pour les plaines du Nord des États-Unis) (ICF, 2013). Parmi les autres facteurs importants, figurent le prix des engrais et des produits végétaux, ainsi que le taux d'intérêt. En outre, comme pour chaque mesure d'atténuation, les études peuvent présenter des divergences en fonction des différentes définitions des mesures d'atténuation et des différentes hypothèses retenues au sujet de variables autres que celles mentionnées ci-avant.

Tableau 6. Estimations du rapport coût-efficacité pour l'apport raisonné de nutrients

\begin{tabular}{|c|c|c|}
\hline Rapport coût-efficacité & Référence & Lieu \\
\hline $\begin{array}{l}<0 \text { EUR/t. } \mathrm{CO}_{2} \mathrm{e} \text {, dispositifs à faible coût sur exploitations } \\
\text { céréalières }>80 \text { ha }\end{array}$ & (Godwin et al., 2003) & Royaume-Uni \\
\hline $\begin{array}{l}<0 \text { EUR/t. } \mathrm{CO}_{2} \mathrm{e} \text {, dispositifs à coût élevé sur exploitations } \\
\text { céréalières }>250 \text { ha }\end{array}$ & (Godwin et al., 2003) & Royaume-Uni \\
\hline $143 \mathrm{EUR} / \mathrm{t} . \mathrm{CO}_{2} \mathrm{e}$ & (Amann et al., 2008) & UE des 27 \\
\hline$(175 \mathrm{EUR}) / \mathrm{t} . \mathrm{CO}_{2} \mathrm{e}$ & (Bates et al., 2009) & UE des 27 \\
\hline $212 \mathrm{EUR} / \mathrm{t} . \mathrm{CO}_{2} \mathrm{e}$ & $\begin{array}{l}\text { (Hoglund-Isaksson et al., } \\
\text { 2010) }\end{array}$ & UE des 27 \\
\hline$<0$ EUR à $39 \mathrm{EUR} / \mathrm{t} . \mathrm{CO}_{2} \mathrm{e}$, exploitations de 400 ha & $(I C F ; 2013)$ & États-Unis \\
\hline 6 EUR à 332 EUR/t. $\mathrm{CO}_{2} \mathrm{e}$, exploitations de $100-200$ ha & $($ ICF ; 2013) & États-Unis \\
\hline
\end{tabular}

* Les données entre parenthèses signalent des économies.

Source : références citées. 


\section{Autres effets}

L'agriculture de précision peut engendrer d'autres avantages que la réduction des émissions de GES. En renforçant le rendement d'utilisation de l'azote, l'agriculture de précision peut amoindrir le lessivage de l'azote et atténuer ainsi la pollution diffuse de l'eau. En diminuant le nombre de passages des machines, elle peut également réduire le tassement du sol, la consommation de carburant et les émissions de GES qui lui sont associées. Lorsqu'elle permet de limiter l'utilisation de produits agrochimiques, l'agriculture de précision peut également contribuer à amoindrir la pollution du sol et de l'eau (Bongiovanni et Lowenberg-Deboer, 2004).

\section{Difficultés de mise en cuvre}

Depuis dix ans, le nombre d'agriculteurs ayant recours à des technologies de précision plus simples ou plus complexes est en augmentation, atteignant 5 à $10 \%$ pour certaines cultures et dans certains pays (producteurs de blé et de maïs aux États-Unis, ensemble des agriculteurs en Allemagne, producteurs céréaliers en Australie). Cependant, compte tenu de l'importance de l'apport de capitaux nécessaire, l'agriculture de précision s'est implantée principalement dans les exploitations d'une superficie supérieure à 200 ha (Diekmann et Batte, 2010 ; ICF, 2013 ; Pedersen et al., 2001 ; Reichardt et al., 2009 ; Robertson et al., 2012). À terme, une tendance à la hausse des prix des cultures et des intrants et une baisse des coûts des technologies pourraient rendre l'agriculture de précision rentable également pour les exploitations de taille moyenne.

Les inconvénients suivants ont été mentionnés principalement par des éleveurs des États-Unis, d'Europe et d'Australie (Diekmann et Batte, 2010 ; Reichardt et al., 2009 ; Reichardt et Jurgens, 2009) : sensibilisation insuffisante, temps nécessaire pour se familiariser avec la technologie, connaissances techniques lacunaires, incompatibilité entre les équipements des différents fabricants, coûts de mise en œuvre (et coûts d'opportunité connexes) élevés, bénéfices difficiles à quantifier.

\subsection{Les inhibiteurs de nitrification}

\section{Introduction}

Les émissions de $\mathrm{N}_{2} \mathrm{O}$ provenant du sol sont la deuxième catégorie la plus importante d'émissions agricoles consignée dans les inventaires des gaz à effet de serre de l'OCDE. Elles proviennent principalement de l'épandage d'azote (de synthèse et organique) sur les grandes cultures et les pâturages, ainsi que du dépôt direct d'azote par les animaux brouteurs. Les inhibiteurs de nitrification peuvent restreindre les émissions de $\mathrm{N}_{2} \mathrm{O}$ provenant du sol en ralentissant la vitesse de conversion de l'ammonium présent dans les engrais en nitrate. En d'autres termes, les émissions d'hémioxyde d'azote reculent sous l'effet de la diminution du taux de conversion des nitrates en hémioxyde d'azote (diazote). Les inhibiteurs de nitrification peuvent atténuer les émissions comme conséquence des phénomènes suivants: a) réduction des émissions directes de $\mathrm{N}_{2} \mathrm{O}$; b) réduction du lessivage et de la volatilisation de l'azote et des émissions indirectes connexes de $\mathrm{N}_{2} \mathrm{O}$; c) amélioration du rendement des cultures et des pâturages et/ou diminution des quantités utilisées d'engrais azotés - la restriction de l'épandage d'azote de synthèse fera reculer les émissions de l'industrie productrice d'engrais (qui seront cependant compensées par l'énergie mise en œuvre pour la fabrication des inhibiteurs de nitrification)-; d) diminution de la consommation d'énergie pour les opérations agricoles en conséquence de la baisse de la fréquence de l'épandage d'engrais (Weiske et al. 2007).

\section{Impact attendu sur les émissions de GES}

L'utilisation d'inhibiteurs de nitrification et d'engrais à libération prolongée a fait l'objet d'études diverses (Smith et al., 1997 ; Merino et al., 2002 ; Di et Cameron, 2003 ; Di et Cameron, 2004 ; Ball et al., 2004; Di et al., 2007). En Nouvelle-Zélande, des travaux précurseurs ont révélé que l'épandage de dicyandiamide (DCD) sur les sols d'herbages pâturés pouvait supprimer jusqu'à $82 \%$ des émissions de 
$\mathrm{N}_{2} \mathrm{O}$ provenant principalement des flaques d'urine (Di et Cameron, 2002) ${ }^{15}$. De même, ces travaux ont démontré que l'épandage de DCD pouvait aussi améliorer la production herbagère et diminuer la concentration d'azote dans les eaux d'assainissement de plus de moitié, ajoutant une incitation d'ordre économique à la réduction des pertes d'azote. Une méthode a été élaborée pour introduire le recours au DCD et aux inhibiteurs d'uréase dans le secteur agricole des inventaires de GES en Nouvelle-Zélande (voir Gouvernement néo-zélandais, 2014 : p. 190-1). Une méta-analyse plus récente a conclu que les inhibiteurs de nitrification pouvaient réduire les émissions de $\mathrm{N}_{2} \mathrm{O}$ à hauteur de $31 \%$ à $44 \%$ (Akiyama et al., 2010). Le tableau 7 présente des estimations des taux de réduction.

Tableau 7. Taux de réduction des émissions associé aux inhibiteurs de nitrification

\begin{tabular}{|c|c|c|c|}
\hline $\begin{array}{c}\text { Forme } \\
\text { d'atténuation }\end{array}$ & Taux de réduction des émissions & Source & Lieu \\
\hline \multirow{6}{*}{$\begin{array}{l}\text { Réduction du } \\
\mathrm{N}_{2} \mathrm{O} \text { provenant } \\
\text { du sol }\end{array}$} & 0.3 t. $\mathrm{CO}_{2} \mathrm{e} / \mathrm{ha} / \mathrm{an}$ & Moran et al. 2008 : p. 45). & Royaume-Uni \\
\hline & Diminution de $51 \%$ du $\mathrm{N}_{2} \mathrm{O}$ provenant du sol & Weiske et al. 2007 : p. 32). & Allemagne \\
\hline & $\begin{array}{l}\text { DCD : diminution de } 20-40 \% \text { du } \mathrm{N}_{2} \mathrm{O} \text { provenant } \\
\text { du sol et de } 6.5 \%-13 \% \text { de l'épandage d'engrais }\end{array}$ & ICF (2008 : p. 48). & $\begin{array}{l}\text { Nouvelle- } \\
\text { Zélande }\end{array}$ \\
\hline & $\begin{array}{l}\text { Agrotain }{ }^{*} \text { : diminution de } 5 \% \text { du } \mathrm{N}_{2} \mathrm{O} \text { provenant } \\
\text { du sol et de } 8 \% \text { de l'épandage d'engrais }\end{array}$ & ICF (2008 : p. 48). & \\
\hline & $\begin{array}{l}\text { DCD : réduction de } 67 \% \text { et } 53 \% \text { de } \mathrm{EF}_{3 \mathrm{PRP}, \mathrm{CPP}} \text { et } \\
\text { Frac }{ }_{L E A C H} \text {, respectivement }\end{array}$ & $\begin{array}{l}\text { Gouvernement néo-zélandais } \\
(2014, \text { p. 190-1) }\end{array}$ & $\begin{array}{l}\text { Nouvelle- } \\
\text { Zélande }\end{array}$ \\
\hline & $\begin{array}{l}\text { Inhibiteurs d'uréase : réduction de } 45 \% \text { de } \\
\text { Frac GASF }\end{array}$ & & \\
\hline \multirow{2}{*}{$\begin{array}{l}\text { Réduction du } \\
\mathrm{N}_{2} \mathrm{O} \text { provenant } \\
\text { du sol }\end{array}$} & Diminution de $30 \%$ du $\mathrm{N}_{2} \mathrm{O}$ provenant du sol & Bates et al. 2009 : 28). & UE des 27 \\
\hline & $\begin{array}{l}\text { Diminution de } 31 \% \text { à } 44 \% \text { du } \mathrm{N}_{2} \mathrm{O} \text { provenant du } \\
\text { sol }\end{array}$ & Akiyama et al. (2010) & Divers \\
\hline $\begin{array}{l}\text { Hausse de la } \\
\text { production }\end{array}$ & Hausse de la production de $7.5 \%$ à $15 \%$ & ICF (2008: 48). & $\begin{array}{l}\text { Nouvelle- } \\
\text { Zélande }\end{array}$ \\
\hline
\end{tabular}

Source : Références cités ; *N-[n-butyl] triamide thiophosphorique.

\section{Éléments des coûts et rapport coût-efficacité}

L'emploi d'inhibiteurs de nitrification ne comporte aucun coût ponctuel (hormis le temps qui peut être nécessaire pour apprendre à l'utiliser efficacement). Le principal coût récurrent est celui de l'achat des inhibiteurs, qui peut varier en fonction de la culture et du type d'inhibiteur (voir tableau 8). Ces coûts sont en partie compensés par les bénéfices récurrents résultant de : a) la réduction de la fréquence de l'épandage des engrais ; b) la réduction de la quantité d'engrais utilisé et/ou l'amélioration du rendement. Le tableau 9 reprend les estimations du rapport coût-efficacité par tonne de $\mathrm{CO}_{2} \mathrm{e}$ en moins.

Le rapport coût-efficacité des inhibiteurs de nitrification est sensible à un certain nombre de facteurs. D'abord, il dépend des conditions climatiques et du type de sol : «le DCD s'avère un tant soit peu moins efficace dans les zones plus chaudes (Edmeades, 2004) et sur les sols acides (de Klein et al., 2001). » ICF (2008 : p. 57), voir également (ICF, 2013 : p. 2.38). Les taux de charge peuvent également jouer un rôle : «il est probable que cette technologie soit faisable (c'est-à-dire, économiquement viable) uniquement sur les sols où le taux de charge est élevé et où la réduction des émissions est de grande envergure » (Schulte et al., $2012:$ p. 13).

15. Le dicyandiamide (DCD) a été retiré volontairement du marché en Nouvelle-Zélande sous la pression des consommateurs, qui craignent que de faibles taux de résidus se retrouvent dans les produits laitiers. De tels taux d'inhibiteurs dans les produits laitiers ne présentent pas de risque pour la santé humaine. 
-RAPPORT COÛT-EFFICACITÉ DES MESURES D’ATTÉNUATION DES ÉMISSIONS DE GAZ À EFFET DE SERRE EN AGRICULTURE

Tableau 8. Estimations des coûts des inhibiteurs de nitrification

\begin{tabular}{llll}
\hline Coûts récurrents & Coût total & Étude & Lieu \\
\hline Total & de 32 EUR à 61 EUR/ha/an & Moran et al. (2008 : p. 48) & Royaume-Uni \\
Achat de NI & Agrotain : 19 EUR/ha/an & ICF $(2008:$ p. 52) & Nouvelle-Zélande \\
& DCD $: 87$ EUR/ha/an & ICF $(2008:$ p. 52) & \\
Achat de NI & NServe $: 15$ EUR/ha/an & ICF $(2013:$ p. 2.40) & \\
& Agrotain :de 4 EUR à 10 EUR/ha/an & ICF $(2013:$ p. 2.40) & \\
\hline
\end{tabular}

Tableau 9. Rapport coût-efficacité estimé des inhibiteurs de nitrification*

\begin{tabular}{|c|c|c|c|}
\hline $\begin{array}{c}\text { Fourchette du rapport } \\
\text { coût-efficacité }\end{array}$ & Unités & Étude & Lieu \\
\hline de 107 à 202 & EUR/t. $\mathrm{CO}_{2} \mathrm{e}$ & Moran et al. 2008 : p. 49). & Royaume-Uni \\
\hline de (19.4) à 9.2 & $\mathrm{EUR} / \mathrm{t} . \mathrm{CO}_{2} \mathrm{e}$ & Weiske et al. (2007) & Allemagne \\
\hline de (1903) à 602 & EUR/t. $\mathrm{CO}_{2} \mathrm{e}$ & ICF (2008 : p. 55) & Nouvelle-Zélande \\
\hline$>55$ & EUR/t. $\mathrm{CO}_{2} \mathrm{e}$ & Amman et al. (2008 : p. 43) & UE des 27 \\
\hline 10 & $\mathrm{EUR} / \mathrm{t} . \mathrm{CO}_{2} \mathrm{e}$ & Bates et al. (2009 : p. 54) & UE des 27 \\
\hline$>450$ & EUR/t. $\mathrm{CO}_{2} \mathrm{e}$ & Schulte et al. (2012 : p. 33) & Irlande \\
\hline de 48 à 296 & EUR/t. $\mathrm{CO}_{2} \mathrm{e}$ & ICF (2013 : p. 2.41) & États-Unis \\
\hline 61 & EUR/t. $\mathrm{CO}_{2} \mathrm{e}$ & Pellerin et al. (2013 : p. 30) & France \\
\hline
\end{tabular}

* Les données entre parenthèse signalent des économies

\section{Autres coûts et avantages}

L'impact des inhibiteurs de nitrification sur l'environnement devrait être compensé par la baisse de la fabrication d'engrais. D'autres bénéfices sont possibles : réduction du tassement du sol grâce à la diminution de la fréquence de l'épandage des engrais (Weiske et al., 2007 : p. 34), amélioration de la qualité de l'eau grâce à l'atténuation du lessivage de l'azote (Schulte et al., 2012). De possibles retombées négatives ont également été mentionnées, notamment l'inhibition de la capacité d'oxydation du méthane contenu dans le sol (Weiske et al., 2007: 34), la toxicité pour les plantes et l'intensification de la volatilisation du $\mathrm{NH}_{3}$ (Bates et al., 2009 : 28).

\section{Difficultés de mise en œuvre et possibilités offertes}

Les coûts peuvent être élevés et l'efficience ainsi que le rapport coût-efficacité sont très variables en fonction de facteurs tels que la catégorie d'inhibiteurs de nitrification, la culture concernée, le type de sol et les conditions climatiques, ce qui impose de cibler précisément les mesures d'accompagnement et l'emploi des inhibiteurs de nitrification afin d'en optimiser la rentabilité. Dans certains pays de l'OCDE (comme le Royaume-Uni), le taux actuel d'utilisation des inhibiteurs de nitrification est faible, ce qui signifie que la progression possible du taux d'adoption devrait s'accompagner d'une marge de réduction du prix d'achat et d'amélioration du rapport coût-efficacité. Enfin, l'analyse de l'atténuation des émissions de GES à mettre au compte de l'utilisation des inhibiteurs de nitrification devrait tenir compte de ses conséquences hors de l'exploitation agricole, qui pourraient être non négligeables, notamment de la baisse des émissions due à la réduction de l'utilisation (et de la production) d'engrais ou à l'accroissement des rendements, qui fera baisser la production (et les émissions) ailleurs. 


\subsection{Le changement d'affectation des terres par boisement des pâturages}

\section{Introduction}

Le taux de séquestration du carbone varie en fonction de l'affectation des terres. En conséquence, encourager (ou, parfois, empêcher) certaines utilisations des terres peut contribuer aux efforts d'atténuation des émissions de GES. Cette étude de cas se concentre sur le boisement des pâturages, qui peut atténuer les émissions de GES par les trois moyens suivants : la séquestration par accroissement des stocks de carbone dans la biomasse aérienne ; la séquestration par accroissement des stocks de carbone dans le sol ; la substitution des carburants et matériaux à forte intensité de carbone (acier, béton) par des produits issus de la forêt.

\section{Impact attendu sur les émissions de GES}

Le tableau 10 présente des estimations des taux de réduction des émissions. La réduction des émissions par séquestration du carbone lors de l'expansion de la forêt est un avantage ponctuel ; une fois sa taille finale atteinte, la forêt cesse de puiser du carbone dans l'atmosphère. À l'inverse, la séquestration du carbone dans la biomasse des produits récoltés se maintient année après année et le carbone peut être stocké durablement dans le bois d'œuvre produit, notamment dans le bois de charpente.

Les résultats du tableau 13 révèlent des variations entre les études et au sein de chacune d'elles. Selon Stavins et Richards (2005:iv), les principaux facteurs affectant le rapport coût-efficacité de la séquestration du carbone dans les forêts sont les suivants : espèces d'arbres concernées ; coût d'opportunité des terres; destination du carbone de la biomasse au brûlage, à la récolte ou aux puits forestiers ; variations anticipées des prix des produits forestiers et agricoles; méthodes d'analyse employées pour rendre compte des flux de carbone dans le temps; taux d'actualisation retenu pour l'analyse ; moyens d'action choisis pour atteindre un objectif donné de séquestration du carbone.

Le coût d'opportunité des terres est particulièrement difficile à prévoir étant donné que la marge brute par hectare peut varier fortement dans l'espace, en fonction de la productivité de la terre. Il peut également varier dans le temps en fonction de facteurs tels que le coût des intrants, le prix des matières premières et le cadre d'action.

En admettant que la croissance de la demande mondiale de produits alimentaires et décarbonation progressive du secteur de l'énergie se poursuivent, le rapport coût-efficacité des solutions de substitution des énergies diminuera sans doute à moyen ou long terme.

Tableau 10. Réduction des émissions associé au boisement des pâturages

\begin{tabular}{|c|c|c|c|}
\hline Forme d'atténuation & Réduction des émissions & Source & Lieu \\
\hline \multirow{4}{*}{$\begin{array}{l}\text { Accroissement du stockage } \\
\text { de carbone dans la } \\
\text { biomasse aérienne et dans } \\
\text { le sol }\end{array}$} & 11.8 t. $\mathrm{CO}_{2} \mathrm{e} / \mathrm{ha} / \mathrm{an}$ & Radov et al. (2007 : p. 37). & Royaume-Uni \\
\hline & 18.4 t. $\mathrm{CO}_{2} \mathrm{e} / \mathrm{ha} / \mathrm{an}$ & Moran et al. (2008) & Royaume-Uni \\
\hline & de 3.7 à 13.2 t. $\mathrm{CO}_{2} \mathrm{e} / \mathrm{ha} / \mathrm{an}$ & ADAS (in Valantin, 2012 : p. 16). & Royaume-Uni \\
\hline & 10.3 t. $\mathrm{CO}_{2} \mathrm{e} / \mathrm{ha} / \mathrm{an}$ & $\begin{array}{l}\text { Sustainable Energy Ireland } \\
(2009: \text { p. 160) }\end{array}$ & Irlande \\
\hline \multirow{4}{*}{$\begin{array}{l}\text { Substitution des carburants } \\
\text { et matériaux à forte intensité } \\
\text { de carbone }\end{array}$} & Carburants : 5.2 t. $\mathrm{CO}_{2} \mathrm{e} / \mathrm{ha} / \mathrm{an}$ & Moran et al. (2008) & Royaume-Uni \\
\hline & Matériaux : & & \\
\hline & 52.6 t. $\mathrm{CO}_{2} \mathrm{e} / \mathrm{ha} / \mathrm{an}$ & Moran et al. (2008) & Royaume-Uni \\
\hline & 6.9 t. $\mathrm{CO}_{2} \mathrm{e} / \mathrm{ha} / \mathrm{an}$ & ADAS (in Valantin, $2012: 16$ ). & Royaume-Uni \\
\hline
\end{tabular}




\section{Éléments des coûts et rapport coût-efficacité}

Les tableaux 11 et 12 fournissent les principaux éléments de coûts et le tableau 13 présente les estimations du rapport coût-efficacité.

Tableau 11. Principaux coûts et avantages ponctuels et récurrents du boisement des pâturages

\begin{tabular}{lll}
\hline & \multicolumn{1}{c}{ Coûts } & \multicolumn{1}{c}{ Bénéfices } \\
\hline Ponctuels & $\begin{array}{l}\text { Création } \\
\text { Perte de valeur foncière } \\
\text { Récolte }\end{array}$ & Vente des produits récoltés \\
Récurrents & $\begin{array}{l}\text { Coupes d'éclaircie } \\
\text { Manque à gagner }\end{array}$ & Vente des produits d'éclaircie \\
\hline
\end{tabular}

Tableau 12. Estimations des coûts et avantages du boisement des pâturages*

\begin{tabular}{|c|c|c|c|}
\hline $\begin{array}{l}\text { Coûts/économies } \\
\text { ponctuels }\end{array}$ & $\begin{array}{c}\text { Coût total } \\
\text { (ou économie totale) }\end{array}$ & Étude & Lieu \\
\hline Coût de création & de 1588 à 3810 EUR/ha & Radov et al. (2007) & Royaume-Uni \\
\hline Coût de création & 1588 EUR/ha & Moran et al. (2008) & Royaume-Uni \\
\hline Coût de création & de 1664 à 6858 EUR/ha & ADAS (in Valantin, 2012 : & Royaume-Uni \\
\hline Coût de création & 3500 EUR/ha & $\begin{array}{l}\text { Sustainable Energy Ireland (2009, } \\
\text { p. 160) }\end{array}$ & Irlande \\
\hline Perte de valeur foncière & de 3175 à 9525 EUR/ha & Radov et al. (2007) & Royaume-Uni \\
\hline Vente du bois récolté & (de 9525 à 10541 EUR/ha) & Moran et al. (2008 : p. 95). & Royaume-Uni \\
\hline $\begin{array}{l}\text { Coûts/économies } \\
\text { récurrents }\end{array}$ & $\begin{array}{c}\text { Coût total } \\
\text { (ou économie totale) }\end{array}$ & Étude & Lieu \\
\hline Entretien (éclaircie, etc.) & $\begin{array}{l}\text { (1 } 905 \text { EUR/ha pour } 4 \text { coupes } \\
\text { d'éclaircie) }\end{array}$ & Moran et al. (2008 : p. 95). & Royaume-Uni \\
\hline Manque à gagner & Très variable, de 64 à 445 EUR/ha & Valantin (2012 : p. 6). & Royaume-Uni \\
\hline Loyer foncier & $300 \mathrm{EUR} / \mathrm{ha} / \mathrm{an}$ & $\begin{array}{l}\text { Sustainable Energy Ireland (2009, } \\
\text { p. 160) }\end{array}$ & Irlande \\
\hline Vente de bois & (87 EUR/ha/an) & $\begin{array}{l}\text { Sustainable Energy Ireland (2009, } \\
\text { p. 160) }\end{array}$ & Irlande \\
\hline
\end{tabular}

* Les données entre parenthèses signalent des économies.

Tableau 13. Rapport coût-efficacité estimé du boisement des pâturages

\begin{tabular}{|c|c|c|c|}
\hline Coûts/économies & $\begin{array}{l}\text { Coût total } \\
\text { (ou économie totale) }\end{array}$ & Étude & Lieu \\
\hline $\begin{array}{l}\text { Séquestration et } \\
\text { substitution }\end{array}$ & (de 9 à 3 EUR/t. $C_{2} \mathrm{e}$ ) & Moran et al. (2008) & Royaume-Uni \\
\hline $\begin{array}{l}\text { Séquestration et } \\
\text { substitution }\end{array}$ & (de 77 EUR) à 131 EUR/t. $\mathrm{CO}_{2} \mathrm{e}$ & ADAS (in Valantin, 2012) & Royaume-Uni \\
\hline $\begin{array}{l}\text { Séquestration et } \\
\text { substitution }\end{array}$ & & Price (in Valantin, 2012) & Royaume-Uni \\
\hline Séquestration & de 39 EUR à 50 EUR/t. $\mathrm{CO}_{2} \mathrm{e}$ & EPA Queensland (2008 : p. 78). & Australie \\
\hline Séquestration & $\begin{array}{l}\text { Valeur actuelle nette = } \\
3 \text { EUR/t. } \mathrm{CO}_{2} \mathrm{e} \\
\text { de } 6 \text { EUR à } 18 \text { EUR/t. } \mathrm{CO}_{2} \mathrm{e} \\
\text { (1997) }\end{array}$ & Stavins et Richards (2005:v) & États-Unis \\
\hline Séquestration & $30 \mathrm{EUR} / \mathrm{t} . \mathrm{CO}_{2} \mathrm{e}$ & $\begin{array}{l}\text { Sustainable Energy Ireland (2009, } \\
\text { p. 79) }\end{array}$ & Irlande \\
\hline
\end{tabular}




\section{Autres coûts et avantages}

Les autres coûts et avantages risquent de dépendre du type de plantation et du mode de gestion. Néanmoins, toute évaluation devrait tenir compte des conséquences du déplacement de la production.

\section{Difficultés de mise en cuvre}

Cette solution peut présenter plusieurs difficultés. Premièrement, l'identification des sites adaptés à la mise en œuvre ne va pas de soi. Deuxièmement, il n'est pas aisé de choisir les taux d'actualisation appropriés pour calculer le rapport coût-efficacité et de chiffrer les conséquences du déplacement ; dans certains cas, le choix peut se porter sur l'agroforesterie afin d'éviter les pertes de production et le déplacement des émissions. Le carbone séquestré risque également d'être libéré dans le cadre de phénomènes tels que les incendies de forêt ou la décomposition du bois d'œuvre produit. Valantin (2012 : p. 7) affirme que ces « risques de non-permanence ne semblent pas avoir été suffisamment pris en compte dans les études précédentes ».

\subsection{La complémentation en lipides dans l'alimentation des ruminants ${ }^{16}$}

\section{Introduction}

La complémentation en lipides est une pratique d'engraissement parmi d'autres (comme, par exemple, la restriction de la teneur en protéines de l'alimentation et les compléments alimentaires du bétail) ayant pour but de réduire les émissions de GES des pratiques d'élevage. Cette mesure repose sur l'augmentation de la quantité de certains ingrédients utilisés couramment pour l'alimentation du bétail. Chez les ruminants, une alimentation traditionnelle contient de $1.5 \%$ à $3 \%$ de matière grasse en pourcentage de la matière sèche (\% MS), en fonction de si l'animal est élevé principalement à l'herbe ou s'il est nourri d'une proportion élevée de concentrés (aliments non fourragers à forte teneur énergétique et protéique, par exemple, mélange de céréales, tourteaux de soja, pulpe de betterave, etc.). L'augmentation de la teneur en matières grasses de l'alimentation permet de réduire les émissions de $\mathrm{CH}_{4}$ produites par fermentation entérique dans le rumen à travers les processus biologiques de l'appareil digestif. La réduction du $\mathrm{CH}_{4}$ est proportionnelle à la teneur en matières grasses, mais, afin d'éviter d'éventuels problèmes de santé et pour des raisons pratiques, la teneur en matières grasses doit être limitée à 5-6\% MS. Diverses sources supplémentaires de lipides existent: céréales entières (colza, lin, soja), huiles végétales (huile de tournesol, huile de colza, huile de palme, huile de coco) et produits spéciaux (Energy Booster, Megalac). Les industries des biocarburants peuvent également fournir des sous-produits à teneur élevée en lipides. Certains éleveurs administrent déjà des compléments en lipides à leurs troupeaux à productivité élevée afin d'amplifier la valeur énergétique de l'alimentation. Or, même pour ces animaux, la teneur totale en matières grasses de l'alimentation ne devrait pas dépasser 5-6\% MS. Le potentiel d'ingestion supplémentaire est encore plus élevé pour les troupeaux ne recevant actuellement aucun complément en lipides, bien que l'ajout de lipides à l'alimentation des animaux nourris principalement de fourrage (pâturage ou herbe fauchée) peut s'avérer difficile, d'un point de vue pratique.

\section{Impact attendu sur les émissions de GES}

Le principal impact de la complémentation en lipides est la réduction des émissions de $\mathrm{CH}_{4}$ issues de la fermentation entérique, dans une proportion égale à la teneur en matières grasses (tableau 14). Deux mécanismes risquent toutefois de contrebalancer partiellement cette atténuation: a) une éventuelle augmentation du $\mathrm{CH}_{4}$ émis par le stockage du fumier (si l'accroissement de la teneur en matières grasses diminue la digestibilité de l'ensemble des aliments, le fumier s'enrichit en matière organique non digérée et les émissions de méthane issues du stockage s'intensifient, bien que les preuves scientifiques de ce phénomène soient limitées) ; b) une éventuelle croissance des émissions liées à la production d'aliments à teneur élevée en matières grasses (alors que les émissions liées à la production des aliments à teneur

16. Cette étude de cas s'appuie en partie sur les travaux de Frelih-Larsen et al. (2014). 
élevée en amidon - ou protéines - peuvent diminuer simultanément). Ce deuxième mécanisme dépend des ingrédients utilisés dans l'alimentation et des pratiques employées pour leur culture ; afin de minimiser les effets négatifs, les éleveurs devraient être incités à utiliser des sources de lipides à faible intensité d'émissions (à savoir, des cultures dont le taux d'émissions de GES par kg de produit est relativement faible). En général, les émissions de GES liées au changement d'affectation des terres sont sensiblement inférieures pour les cultures poussant en Europe (lin, colza oléagineux) que pour celles des régions tropicales (coco, huile de palme ou soja).

Tableau 14. Réduction des émissions associée à l'élévation de la teneur en matières grasses de l'alimentation des ruminants à $5 \% \mathrm{MS}$

\begin{tabular}{|c|c|c|c|}
\hline $\begin{array}{c}\text { Forme } \\
\text { d'atténuation }\end{array}$ & $\begin{array}{c}\text { Réduction } \\
\text { des émissions }\end{array}$ & Référence & Lieu \\
\hline $\begin{array}{l}\text { Émissions de } \mathrm{CH}_{4} \text { issues de } \\
\text { la fermentation entérique }\end{array}$ & $\begin{array}{l}\text { Pour } 1 \% \text { de matière grasse } \\
\text { ajoutée, les émissions de } \\
\mathrm{CH}_{4} \text { sont réduites d'environ } \\
4 \% \text { pour l'ensemble des } \\
\text { ruminants }\end{array}$ & $\begin{array}{l}\text { Beauchemin et al. (2008), } \\
\text { Grainger et Beauchemin (2011), } \\
\text { Eugene et al. (2008), Moate et } \\
\text { al. (2011), Martin et al. (2010), } \\
\text { Doreau et al. (2014), Pellerin et } \\
\text { al. (2013) }\end{array}$ & Pays développés \\
\hline $\begin{array}{l}\text { Changement d'affectation } \\
\text { des terres }\end{array}$ & $\begin{array}{l}\text { Bétail : de }+100 \mathrm{~kg} \text { à }+191 \\
\mathrm{~kg} \mathrm{CO} 2 \mathrm{e} / \text { animal/an }\end{array}$ & $\begin{array}{l}\text { Pellerin et al. (2013 : p. } 59) \text { - à } \\
\text { partir des données standard de } \\
\text { l'ACV compilées par Dia'terre® } \\
\text { - Ges'tim et l'INRA. }\end{array}$ & $\begin{array}{l}\text { Mondial, lié à } \\
\text { l'alimentation du bétail } \\
\text { en France }\end{array}$ \\
\hline
\end{tabular}

\section{Éléments des coûts et rapport coût-efficacité}

Dans la plupart des cas, les éleveurs risquent de devoir supporter une hausse du coût de l'alimentation en raison de l'achat d'oléagineux, de sous-produits ou d'huiles, généralement plus coûteux que les ingrédients concentrés qu'ils remplacent - de fortes variations risquent toutefois de se manifester entre les ingrédients (dans une même région), entre les régions (pour un même ingrédient) et dans le temps. Les estimations du rapport coût-efficacité fluctuent entre une économie nette de $70 \mathrm{EUR} / \mathrm{t} . \mathrm{CO}_{2} \mathrm{e}$ et un coût d'environ $500 \mathrm{EUR} / \mathrm{t} . \mathrm{CO}_{2} \mathrm{e}$ (tableau 15). Les hypothèses relatives aux coûts des aliments du bétail expliquent en grande partie cette variabilité (Bates (2001), par exemple, réalise une estimation des économies nettes en partant de l'hypothèse que le prix de la ration n'augmentera pas).

Tableau 15. Estimations du rapport coût-efficacité de la complémentation en lipide dans l'alimentation des ruminant*

\begin{tabular}{|c|c|c|}
\hline Rapport coût-efficacité & Référence & Lieu \\
\hline (70 EUR/t. $\left.\mathrm{CO}_{2} \mathrm{e}\right)$ & (Bates, 2001) & UE des 15 \\
\hline (69 EUR/t. $\left.\mathrm{CO}_{2} \mathrm{e}\right)$ & (Graus et al., 2004) & Monde \\
\hline de 499 EUR à 508 EUR/t. $\mathrm{CO}_{2} \mathrm{e}$ & $($ ICF ; 2008) & Nouvelle-Zélande \\
\hline de 137 EUR à 262 EUR/t. $\mathrm{CO}_{2} \mathrm{e}$ & (Bates et al., 2009) & UE des 27 \\
\hline de 79 EUR à 158 EUR/t. $\mathrm{CO}_{2} \mathrm{e}$ & (Henderson, à paraître) & Monde \\
\hline de 223 EUR à 335 EUR/t. $\mathrm{CO}_{2} \mathrm{e}$ & (Pellerin et al., 2013) & France \\
\hline
\end{tabular}

\section{Autres effets}

L'ajout de matière grasse peut stimuler la croissance et le rendement laitier. La composition et la teneur en matières grasses du lait peuvent également s'améliorer (Grainger et Beauchemin, 2011 ; Marette et Millet, 2014 ; Patra, 2012). Le tableau 15 ne rend pas compte de cet avantage, qui pourrait accroître le rapport coût-efficacité.

Cependant, si la teneur en matières grasses dépasse 5-6\% MS, le fonctionnement du rumen peut s'altérer, au détriment du rendement laitier et du rythme de la croissance (Grainger et Beauchemin, 2011 ; Pellerin et al., 2013). En outre, les ingrédients à teneur élevée en matières grasses originaires des zones 
tropicales peuvent être associés à une diminution de la biodiversité liée au changement d'affectation des terres.

\section{Difficultés de mise en œuvre}

Réglementer un changement d'alimentation impliquant une réorganisation des ingrédients utilisés en alimentation animale n'est pas chose aisée tant que les avantages économiques ne sont pas clairs. Les programmes visant à soutenir uniquement le supplément de matières grasses qui s'ajoute à la teneur que l'éleveur aurait déjà pu appliquer pour améliorer ses performances sont difficiles à mettre en œuvre, tandis que les approches réglementaires peuvent se heurter à des problèmes de suivi. Pour commencer, la diffusion d'informations peut sensibiliser les agriculteurs et les inciter à planifier des objectifs nutritionnels.

Les obstacles possibles à la mise en œuvre de cette mesure comprennent les problèmes pratiques mentionnés ci-dessus (pour les régimes alimentaires comprenant moins de $20 \%$ MS de concentrés ou ne reposant pas sur une ration totale mixte, la teneur élevée en matières grasses peut présenter des difficultés liées au stockage et au mélange) (Pellerin et al., 2013) et le coût des ingrédients nécessaires (Hristov et al., 2013 ; Pellerin et al., 2013). Autre incertitude : la persistance de l'effet d'atténuation sur le long terme n'a pas encore été vraiment démontrée (Hristov et al., 2013; Martin et al., 2010).

\subsection{La réduction du $\mathrm{CH}_{4}$ issu de la fermentation entérique par amélioration génétique}

\section{Introduction}

L'amélioration génétique du bétail est un processus continu qui apporte des améliorations en châne aux performances des animaux. Les éleveurs peuvent profiter des progrès génétiques par l'achat d'animaux reproducteurs ou de liquide séminal. Dans de nombreux pays, l'industrie utilise des indices de valeur génétique afin de les aider dans ce processus. Un indice de valeur génétique est une valeur numérique globale tenant compte de plusieurs caractéristiques, qui met plus ou moins l'accent sur la productivité (le rendement) et la valeur sélective (longévité, fertilité, etc.) (Miglior et al., 2005). L'amélioration génétique commerciale est axée sur l'efficience économique, qui est étroitement liée à la productivité et à la valeur sélective de l'animal. Une meilleure productivité implique l'utilisation de moins d'intrants pour la même quantité produite, ce qui restreint le volume de déchets, notamment de gaz à effet de serre. En effet, cet objectif d'amélioration génétique a déjà réduit indirectement les émissions de gaz à effet de serre et d'ammoniac des diverses espèces (tableau 16) et devrait produire, à terme, d'autres bénéfices pour l'environnement, selon une étude britannique (Genesis Faraday, 2008).

L'amélioration génétique peut atténuer les émissions de gaz à effet de serre de trois façons (Wall et al., 2010) :

- par effet indirect, en améliorant la productivité et l'efficience des animaux (augmentation de la quantité de lait produite par le même nombre de vaches);

- par effet indirect, en améliorant l'efficience du troupeau de menu et gros bétail (réduction du nombre de recrues en raison de la baisse du nombre d'abattages d'animaux déficients, par exemple);

- par réduction directe des émissions de $\mathrm{CH}_{4}$ issues de la fermentation entérique (pour les ruminants, notamment les bovins et les ovins, voir par exemple http://www.nzagrc.org.nz/media.html).

Les deux premiers effets font déjà partie des objectifs commerciaux d'amélioration génétique, comme on l'a vu ci-avant. Néanmoins, les progrès des effets sur l'environnement peuvent encore se poursuivre et s'accélérer, en particulier en obtenant de meilleurs taux d'amélioration génétique (grâce à la mise au point de nouvelles techniques, l'amélioration des techniques de mesure et d'enregistrement, l'optimisation des mécanismes d'amélioration génétique, etc., voir Genesis Faraday, 2008). Privilégier les indices d'amélioration génétique relatifs à la fertilité et à la valeur sélective peut également amener des progrès. Enfin, la diffusion plus large et plus rapide des améliorations génétiques dans les élevages commerciaux peut avoir des retombées appréciables. Au Royaume-Uni, par exemple, où le cheptel laitier national 
connaît des améliorations génétiques indéniables, l'utilisation des indices et l'amélioration génétique des bovins de boucherie progressent beaucoup plus lentement (Moran et al., 2008).

Les avantages économiques du troisième effet (recours à la sélection génétique pour réduire les émissions de $\mathrm{CH}_{4}$ ) ne sont pas si manifestes. Ces dernières années, les objectifs en matière d'élevage ont commencé d'intégrer certains aspects sociétaux, comme le bien-être et la biodiversité (Wall et al., 2010). Il serait souhaitable d'y inclure aussi la réduction des émissions de $\mathrm{CH}_{4}$, mais cela pourrait nécessiter un soutien des pouvoirs publics.

Tableau 16. Réduction des émissions avec l'amélioration génétique pour réduire les émissions de $\mathrm{CH}_{4}$ liées à la fermentation entérique

\begin{tabular}{|c|c|c|c|}
\hline $\begin{array}{c}\text { Forme } \\
\text { d'atténuation }\end{array}$ & $\begin{array}{c}\text { Réduction } \\
\text { des émissions }\end{array}$ & Référence & Lieu \\
\hline \multirow{2}{*}{$\begin{array}{l}\text { Toutes les } \\
\text { émissions de GES } \\
\text { (méthode de l'ACV, } \\
\text { par produit) }\end{array}$} & $\begin{array}{l}\text { Sur } 20 \text { ans (de } 1988 \text { à 2008), l'intensité des } \\
\text { émissions a chuté de } 15-25 \% \text { pour le poulet, le porc } \\
\text { et les produits laitiers }\end{array}$ & $\begin{array}{l}\text { (Genesis Faraday, } \\
2008 \text { ) }\end{array}$ & Royaume-Un \\
\hline & $\begin{array}{l}\text { Sur } 20 \text { ans (de } 1988 \text { à 2008), l'intensité des } \\
\text { émissions a chuté de } 1 \% \text { pour le bœuf et le } \\
\text { mouton }^{\mathrm{a}}\end{array}$ & $\begin{array}{l}\text { (Genesis Faraday, } \\
\text { 2008) }\end{array}$ & Royaume-Uni \\
\hline \multirow{3}{*}{$\begin{array}{l}\text { Émissions de } \mathrm{CH}_{4} \\
\text { issues de la } \\
\text { fermentation } \\
\text { entérique } \\
\text { (ruminants) }\end{array}$} & Réduction fixée à $14 \%$ (par animal) sur 10 ans & (ICF ; 2008) & $\begin{array}{l}\text { Nouvelle- } \\
\text { Zélande }\end{array}$ \\
\hline & Réduction fixée à $5 \%$ (par troupeau) sur 10 ans & (Bates et al., 2009) & UE-27 \\
\hline & $\begin{array}{l}\text { Aucune incidence sur les émissions de } \mathrm{CH}_{4} \text {, mais } \\
\text { hausse du rendement laitier fixée à } 22.5 \% \text { sur } \\
15 \text { ans }\end{array}$ & (Moran et al., 2008) & Royaume-Uni \\
\hline
\end{tabular}

Source: a : La baisse de ces émissions sur le long terme ne s'explique pas seulement par l'amélioration génétique (atténuation issue de modifications de la gestion des exploitations, notamment de la conduite de l'alimentation et de la gestion des troupeaux).

\section{Impact attendu sur les émissions de GES}

Les effets sur les émissions de GES varieront selon les espèces et la part des différents caractères dans l'amélioration génétique obtenue. Pour les non ruminants (porcins, volailles), l'amélioration de la productivité et de l'efficience devrait entraîner une réduction globale des émissions (de $\mathrm{N}_{2} \mathrm{O}$ et de $\mathrm{CH}_{4}$ ). Les émissions de $\mathrm{CH}_{4}$ issues de la fermentation entérique des ruminants devraient aussi accuser une baisse, qui sera d'autant plus importante que les efforts d'amélioration génétique porteront sur la réduction des émissions de méthane.

\section{Éléments des coûts et rapport coût-efficacité}

Le coût de cette mesure au niveau de l'exploitation est généralement estimé être nul. Même si l'amélioration génétique demandait de substituer la saillie naturelle par l'insémination artificielle, aucune hausse des coûts significative n'aurait probablement lieu (Jacobsen, 2010). En revanche, les progrès de l'efficience (meilleur rendement et/ou diminution des ressources nécessaires) accroîtront les avantages économiques. Par conséquent, la plupart des estimations du rapport coût-efficacité sont négatives (tableau 17). 
Tableau 17. Estimations du rapport coût-efficacité avec l'amélioration génétique pour réduire les émissions de $\mathrm{CH} 4$ liées à la fermentation entérique*

\begin{tabular}{|c|c|c|}
\hline Rapport coût-efficacité & Référence & Lieu \\
\hline de (4 576 EUR) à 0 EUR/t. $\mathrm{CO}_{2} \mathrm{e}$ (fertilité et productivité) & (Moran et al., 2008) & Royaume-Uni \\
\hline $\begin{array}{l}\text { de } 11 \text { EUR à } 18 \text { EUR/t. CO2 (émissions inférieures de } \\
\mathrm{CH}_{4} \text { ) (hypothèse d'un coût de } 12 \text { EUR/animal) }\end{array}$ & $($ ICF ; 2008) & Nouvelle-Zélande \\
\hline $0 \mathrm{EUR} / \mathrm{t} . \mathrm{CO}_{2} \mathrm{e}$ & (Bates et al., 2009) & UE-27 \\
\hline $\begin{array}{l}\text { de (EUR } 4576) \text { à (128 EUR)/t. } \mathrm{CO}_{2} \mathrm{e} \text { (fertilité et } \\
\text { productivité) }\end{array}$ & (MacLeod et al., 2010b) & Royaume-Uni \\
\hline (48 EUR/t. $\left.\mathrm{CO}_{2} \mathrm{e}\right)$ & (Graus et al., 2004) & Monde \\
\hline
\end{tabular}

\section{Autres effets}

Les autres effets possibles concernent l'équilibre coût-bénéfice. Premièrement, l'amélioration de la productivité s'accompagne de co-bénéfices pour l'environnement (atténuation des émissions d'ammoniac et de la pollution diffuse de l'eau, notamment). Deuxièmement, un meilleur rendement risque de demander davantage de concentrés pour le bétail, ce qui peut entraîner un changement négatif d'affectation de terres jusqu'alors consacrées au pâturage, pour qu'elles deviennent labourables (Moran et al., 2008). Troisièmement, s'il est nécessaire d'intensifier l'élevage en espace clos pour obtenir les gains d'efficience possibles, le bien-être des animaux risque d'être mis à mal. Il existe enfin un risque de maladaptation. Mettre l'accent sur l'efficience et la baisse des émissions de GES peut nuire à la diversité génétique et aller à l'encontre des efforts visant à obtenir des races plus rustiques et plus résistantes au changement des conditions climatiques et aux risques accrus de maladies.

\section{Difficultés de mise en ouvre}

Pour élargir et accélérer la diffusion des progrès génétiques dans les troupeaux commerciaux, des campagnes pourraient être lancées pour sensibiliser les agriculteurs aux avantages des indices d'amélioration génétique et, lorsque cette pratique n'est pas courante, de l'insémination artificielle. Dans certains pays, la faible rentabilité pourrait faire obstacle à l'adoption de telles pratiques.

Le délai nécessaire à l'implantation généralisée, dans les cheptels nationaux, d'animaux améliorés génétiquement, en particulier du point de vue des émissions de $\mathrm{CH}_{4}$, est un critère important à prendre en compte. Une fois créées des mesures d'incitation commerciales ou gouvernementales, un certain temps est nécessaire à l'arrivée sur le marché des animaux reproducteurs et du liquide séminal des races peu émettrices de $\mathrm{CH}_{4}$ et à leur utilisation par les éleveurs. L'incidence de la mesure sur le taux de réduction de la pollution et son application sont donc susceptibles de progresser lentement au cours des décennies.

Les obstacles possibles à la mise en œuvre comprennent le manque d'information/de motivation qui peuvent freiner l'adoption d'améliorations génétiques dans certains pays (dont le profil social ou démographique du secteur de la production bovine est spécifique) et le fait que les éleveurs puissent considérer que la baisse des émissions de $\mathrm{CH}_{4}$ n'ajoute aucune valeur économique.

\subsection{La digestion anaérobie}

\section{Introduction}

La digestion anaérobie est une technique de conversion de la matière organique en biogaz (composé de $\mathrm{CH}_{4}$, de dioxyde de carbone et d'autres gaz) ensuite brûlé pour produire de l'électricité ou de la chaleur. Dans l'agriculture, les déjections animales sont une bonne matière de base pour ce processus, de même que les déchets alimentaires, les cultures énergétiques et les résidus de récolte. Outre la production d'énergie renouvelable, la digestion anaérobie du fumier présente l'avantage de réduire considérablement 
les émissions de $\mathrm{CH}_{4}$ lors du stockage, étant donné que la plupart du $\mathrm{CH}_{4}$ est capté puis transformé en dioxyde de carbone par la combustion ${ }^{17}$. Le digestat peut servir d'engrais.

Différentes solutions technologiques existent, dont le choix repose en partie sur le type de fumier présent sur l'exploitation, les installations existantes, la zone climatique considérée (l'efficience du $\mathrm{CH}_{4}$ dans les bassins de fermentation non chauffés peut être très faible à basse température) et la capacité d'investissement. D'autres matières organiques, principalement des produits végétaux tels que le maïs (Kalamaras et Kotsopoulos, 2014), sont souvent digérées avec le fumier car ces matières de base fournissent généralement un meilleur rendement en $\mathrm{CH}_{4}$.

Un digesteur anaérobie peut être construit pour une seule exploitation ou pour centraliser le traitement des déjections animales de plusieurs élevages si la densité de la production animale le permet.

\section{Impact attendu sur les émissions de GES}

La digestion anaérobie réduit les émissions de $\mathrm{CH}_{4}$ du purin stocké en captant le biogaz. Le volume de la réduction des émissions de GES est fonction du volume des émissions du stockage initial, qui dépend à son tour du type de stockage, de la température et de l'exposition de l'installation à l'air libre ou non. S'ajoute à cette réduction, les émissions indirectement évitées grâce au remplacement d'énergies fossiles ; leur importance dépendra du mix énergétique utilisé pour produire de l'électricité dans le pays considéré (voir tableau 18).

Tableau 18. Réduction des émissions avec la digestion anaérobie

\begin{tabular}{llll}
\hline $\begin{array}{l}\text { Forme } \\
\text { d'atténuation }\end{array}$ & $\begin{array}{l}\text { Réduction des } \\
\text { émissions }\end{array}$ & Référence & Lieu \\
\hline $\begin{array}{l}\mathrm{CH}_{4} \text { émis par le } \\
\text { stockage du purin }\end{array}$ & $85 \%$ & (EPA, 2013) & États-Unis \\
& $90 \%$ & (Bates et al., 2009) & UE des 27 \\
& $\begin{array}{l}75-93 \% \text {, diminue } \\
\text { avec la baisse des } \\
\text { températures } \\
\text { annuelles. }\end{array}$ & $\begin{array}{l}\text { Calculs personnels basés sur les facteurs d'émissions } \\
\text { du GIEC (GIEC 2006), les pertes lors du stockage } \\
\text { préalable à la digestion, estimés à 20 \%, les fuites, } \\
\text { estimées à 3 \%, l'efficience de la production nette } \\
\text { d'électricité, estimée à 24 \%, et le facteur d'émission } \\
\text { marginale pour la production électrique, estimé à } \\
0.33 \mathrm{~kg} \mathrm{CO} \mathrm{CO}_{2} \mathrm{e} / \mathrm{kWh}\end{array}$ & $\begin{array}{l}\text { Génpéré } \\
\text { temat }\end{array}$ \\
& & &
\end{tabular}

\section{Éléments des coûts et rapport coût-efficacité}

La digestion anaérobie est une technologie à forte intensité de capital, dont les coûts initiaux élevés ne sont rentables que pour les plus grands élevages ou si plusieurs exploitations adjacentes centralisent le traitement de leur fumier. Outre l'investissement initial, les frais annuels de fonctionnement et d'entretien peuvent être importants en raison de la complexité du système. Néanmoins, la vente de l'énergie produite ou la vente du biogaz est source de revenus. La chaleur produite peut également servir à réduire les dépenses énergétiques de l'exploitation. Le coût d'une infrastructure de distribution de chaleur peut également être un facteur prépondérant de la rentabilité.

L'investissement initial coûterait entre $2700 \mathrm{EUR} / \mathrm{kW}$ et $11000 \mathrm{EUR} / \mathrm{kW}$, alors que les frais de fonctionnement sont estimés représenter entre $2 \%$ et $6 \%$ de cet investissement initial (Hardtlein et Eltrop, 2013 ; Lazarus et Rudstrom, 2007 ; MacLeod et al., 2010 ; Pellerin et al., 2013 ; The Andersons Centre, 2010 ; Walla et Schneeberger, 2008 ; Yiridoe et al., 2009).

Le rapport coût-efficacité est fortement tributaire d'un éventail de facteurs, notamment: «la dimension de l'opération, la méthode de stockage du fumier, le prix de l'électricité, les dépenses d'énergie de l'exploitation (chauffage), la capacité à vendre l'électricité non utilisée pour la production, et-s'il

17. Le potentiel de réchauffement planétaire du $\mathrm{CH}_{4}$ équivaut à 25 fois celui du $\mathrm{CO}_{2}$ sur une période de 100 ans (GIEC, 2007). 
existe un marché d'échange de droits d'émission de carbone - le prix du carbone » La matière de base supplémentaire utilisée dans le digesteur est également un facteur important, ainsi que la zone climatique où il se trouve. Ces facteurs et cette variabilité des données concernant les coûts expliquent en partie la disparité des estimations du rapport coût-efficacité (tableau 19).

Tableau 19. Estimations du rapport coût-efficacité avec la digestion anaérobie*

\begin{tabular}{|c|c|c|}
\hline Rapport coût-efficacité & Référence & Lieu \\
\hline \multicolumn{3}{|l|}{ Digesteurs centralisés } \\
\hline $14 \mathrm{EUR} / \mathrm{t} . \mathrm{CO}_{2} \mathrm{e}$ & (Moran et al. 2008). & Royaume-Uni \\
\hline de 0 EUR à 153 EUR/t. $\mathrm{CO}_{2} \mathrm{e}$ & Amann et al. 2008). & UE des 27 \\
\hline de (48 EUR) à 130 EUR/t. $\mathrm{CO}_{2} \mathrm{e}$ & Bates et al. 2009). & UE des 27 \\
\hline 0 EUR/t. $\mathrm{CO}_{2} \mathrm{e}$ & (MacLeod et al. 2010b) & Royaume-Uni \\
\hline \multicolumn{3}{|l|}{$\begin{array}{l}\text { Digesteurs propres à une seule exploitation } \\
\text { (toutes tailles d'exploitations confondues) }\end{array}$} \\
\hline & (Weiske et Michel 2007) & Allemagne \\
\hline de (2 EUR) à $24 \mathrm{EUR} / \mathrm{t} . \mathrm{CO}_{2} \mathrm{e}$ & (Moran et al. 2008). & Royaume-Uni \\
\hline de (20 EUR) à 62 EUR/t. $\mathrm{CO}_{2} \mathrm{e}$ & $(I C F, 2008)$ & Nouvelle-Zélande \\
\hline $158 \mathrm{EUR} / \mathrm{t} . \mathrm{CO}_{2} \mathrm{e}$ & (Smith et al. 2008). & Monde \\
\hline de 77 EUR à 214 EUR/t. $\mathrm{CO}_{2} \mathrm{e}$ & Bates et al. 2009). & UE des 27 \\
\hline de 22 EUR à 42 EUR/t. $\mathrm{CO}_{2} \mathrm{e}$ & (MacLeod et al. 2010b) & Royaume-Uni \\
\hline de 8 EUR à 693 EUR/t. $\mathrm{CO}_{2} \mathrm{e}$ & $\begin{array}{l}\text { (Hoglund-Isaksson et al. } \\
\text { 2010) }\end{array}$ & UE des 27 \\
\hline $150 \mathrm{EUR} / \mathrm{t} . \mathrm{CO}_{2} \mathrm{e}$ & (Schulte et al. 2012). & Irlande \\
\hline $17 \mathrm{EUR} / \mathrm{t} . \mathrm{CO}_{2} \mathrm{e}$ & (Pellerin et al. 2013). & France \\
\hline de 0 EUR à 635 EUR/t. $\mathrm{CO}_{2} \mathrm{e}$ & (ICF 2013) & États-Unis \\
\hline
\end{tabular}

* Les données entre parenthèses signalent des économies.

Autres effets

La digestion anaérobie présente d'autres avantages : lutte contre la pollution olfactive, destruction des agents pathogènes et des mauvaises herbes présents dans le fumier et amélioration de la gestion du lisier.

Néanmoins, la digestion anaérobie risque d'entraîner une augmentation de la culture des matières de base aux dépens des pâturages ou des aires naturelles, à laquelle s'ajouterait le transport de grands volumes de fumier non transformé et de digestat vers et depuis les installations centralisées.

\section{Difficultés de mise en æuvre}

La généralisation de la mise en œuvre de la digestion anaérobie a été favorisée par divers moyens d'action : aides à l'investissement pour financer les dépenses d'équipement, soutien des prix de l'électricité vendue. La digestion anaérobie pourrait également bénéficier de crédits carbone étant donné que le suivi des économies de gaz à effet de serre est possible à un coût relativement faible. Le nombre élevé d'installations de digestion anaérobie en Allemagne, par rapport aux autres pays d'Europe et du reste du monde, s'explique par un cadre réglementaire favorable (Wilkinson, 2011).

Parallèlement, plusieurs obstacles s'opposent à la mise en œuvre de cette pratique. En particulier, le grand apport de capitaux nécessaire et l'absence d'infrastructures permettant d'injecter l'électricité produite dans le réseau national impliquent des coûts externes importants. Les risques réglementaires et commerciaux concernant les subventions, les conditions de vente de l'électricité par les éleveurs et les prix de l'électricité peuvent également jouer un rôle négatif. Le savoir-faire nécessaire à l'entretien des installations peut décourager les éleveurs. La compatibilité avec les autres réglementations (notamment la 
Directive sur les nitrates de l'UE) et les restrictions éventuelles à l'utilisation de certaines matières de base (comme les déchets alimentaires) peuvent également avoir une incidence.

\subsection{L'amélioration de l'efficacité énergétique des engins agricoles ${ }^{18}$}

Les exploitations agricoles ont besoin d'énergie pour des activités très diverses : alimentation des engins et machines agricoles, gestion du milieu (chauffage, climatisation, éclairage, ventilation, contrôle de l'humidité, etc.), transformation (réfrigération du lait ou séchage des céréales), irrigation et drainage. Les émissions imputables à la consommation d'énergie des exploitations représentent une proportion limitée mais non négligeable du total des émissions de GES d'origine agricole dans les pays de l'OCDE. Des études récentes (AgriClimateChange, 2013; AEA/FEC Services Ltd, 2010) ont mis en lumière l'importance des émissions des engins et machines agricoles en particulier.

\section{Impact attendu sur les émissions de GES}

Selon AgriClimateChange (2013), la proportion des émissions totales de GES imputables aux engins agricoles au niveau des exploitations se situe entre $37 \%$ dans les vignobles et $5 \%$ dans les élevages bovins et les exploitations rizicoles. Les économies de combustibles qui pourraient être réalisées figurent dans la base de données du programme Efficient 20 et sont reprises dans le tableau 20.

Pellerin et al. (2013 : p. 78) estiment que la réduction de la consommation de carburant atteindrait $10 \%$ si les moteurs subissaient des tests (consistant à «faire passer les tracteurs sur un banc d'essai de moteurs afin de pouvoir en optimiser les réglages », Pellerin et al., 2013: p. 75) et $20 \%$ grâce à l'écoconduite. Le taux réel de réduction de la consommation de carburant dépend de l'exploitation, car il est fonction d'une gamme de facteurs tels que le type de culture, le type de sols, le mode de culture et l'efficience de départ du tracteur (Efficient 20, 2013 : p. 3).

Tableau 20. Économies moyennes de carburant réalisables en modifiant l'utilisation des engins agricoles, selon la base de données Efficient 20 (Lieu : UE)

\begin{tabular}{|c|c|c|}
\hline Action & Synthèse & $\begin{array}{l}\text { \% moyen de réduction de la } \\
\text { consommation de carburant }\end{array}$ \\
\hline $\begin{array}{l}\text { Limiter l'utilisation du } \\
\text { tracteur. }\end{array}$ & $\begin{array}{l}\text { Remplacer les tracteurs par des machines à meilleur } \\
\text { rendement énergétique dès que possible }\end{array}$ & 13.0 \\
\hline Adopter l'écoconduite. & $\begin{array}{l}\text { Conduite plus souple, maintien de la vitesse la plus } \\
\text { efficiente, etc. }\end{array}$ & 5.4 \\
\hline $\begin{array}{l}\text { Utiliser une prise de } \\
\text { force économique. }\end{array}$ & $\begin{array}{l}\text { Utiliser une prise de force qui économise le carburant en } \\
\text { permettant au moteur du tracteur de tourner à un moins } \\
\text { grand nombre de tours par minute. }\end{array}$ & 15.4 \\
\hline $\begin{array}{l}\text { Allonger les séquences } \\
\text { de travail. }\end{array}$ & $\begin{array}{l}\text { Plus le champ est long, moins les engins consomment de } \\
\text { carburant pour manœuvrer sur les tournières. }\end{array}$ & 11.0 \\
\hline Adapter le poids. & $\begin{array}{l}\text { Ajuster le lest afin de réduire le dérapage des pneus et le } \\
\text { tassement du sol. }\end{array}$ & 5.7 \\
\hline $\begin{array}{l}\text { Adapter les réglages de } \\
\text { l'outillage. }\end{array}$ & Par exemple, régler la charrue à la profondeur optimale. & 16.8 \\
\hline Bien gérer les pneus. & Choisir le type de pneus approprié, la bonne pression, etc. & 10.7 \\
\hline
\end{tabular}

Source : Efficient 20 (2013) : 6 .

18. Cette étude de cas s'appuie en partie sur les travaux de Frelih-Larsen et al. (2014). 


\section{Éléments des coûts et rapport coût-efficacité}

Les coûts ponctuels de ces modifications sont les suivants : a) délai d'apprentissage nécessaire pour améliorer l'efficacité énergétique ; b) achat de matériel ; c) modification du matériel existant. Des coûts récurrents peuvent intervenir, correspondant au temps passé pour notamment : effectuer les opérations agricoles, contrôler la consommation de carburant ou assister à d'autres formations, et entretenir les machines (voir tableau 21).

Tableau 21. Coûts et économies récurrents associés à l'amélioration de l'efficacité énergétique des engins agricoles (+ : coût ; - : économie)

\begin{tabular}{lcc}
\hline \multicolumn{1}{c}{ Coûts/économies récurrents } & Étude & Lieu \\
\hline - Baisse des dépenses de carburant de $5.4 \%$ à $16.8 \%$ & Efficient $20(2013:$ p. 6$)$ & UE \\
$\begin{array}{l}\text { + Risque que certaines modifications (notamment les techniques } \\
\text { d'écoconduite) puissent allonger la durée des opérations si elles ne } \\
\text { sont pas bien maîtrisées. }\end{array}$ & Efficient $20(2013:$ p. 8). & UE \\
\hline
\end{tabular}

Les éléments dont on dispose laissent penser que le coût net de l'amélioration de l'efficacité énergétique des engins agricoles est susceptible d'être négatif (c'est-à-dire, que les économies de carburant devraient largement compenser les dépenses) (AEA/FEC Services Ltd, 2010, Domingo et al., 2014 : p. 15 ; Pellerin et al., 2013 : p. 78).

\section{Autres coûts et avantages}

Les autres coûts et avantages dépendront du choix des moyens retenus pour améliorer l'efficience. Par exemple, s'il est décidé de diminuer la pression des pneus, le tassement du sol pourrait être atténué, au bénéfice de la production et de l'environnement. D'une façon générale, les émissions en amont correspondant à la production et au transport de carburant, devraient diminuer.

\section{Difficultés de mise en ouvre}

Plusieurs obstacles éventuels s'opposent à l'amélioration de l'efficacité énergétique (AEA/FEC Services Ltd, 2010: Efficient 20 (2013: p. 8). Cependant, en la comparant à d'autres mesures d'atténuation des émissions de GES, Domingo et al. (2014 : p. 16) ont conclu que les mesures d'économie de carburant devraient être faciles à mettre en œuvre pour les agriculteurs et «pourraient représenter la mesure la mieux acceptée par la communauté des exploitants agricoles ».

L'effet rebond est un problème qui nuit à l'efficacité énergétique en général (voir Sorrell, 2007). Il ne devrait pas être particulièrement préoccupant dans le cadre de l'utilisation d'engins agricoles, pour laquelle le bénéfice marginal produit par une utilisation supplémentaire devrait probablement décroitre rapidement au-delà d'un certain taux d'utilisation. Néanmoins, AEA/FEC Services Ltd (2010 : p. 12) préviennent que «les améliorations du rendement énergétique des moteurs des véhicules ne se traduisent pas systématiquement par une baisse de la consommation de carburant ».

En général, le rapport coût-efficacité des économies d'énergie risque de faiblir à mesure que l'intensité des émissions issues de la consommation d'énergie (à savoir, les émissions par unité produite) diminue grâce à l'introduction d'équipements de meilleur rendement énergétique et à la décarbonation de la production d'électricité (AEA/FEC Services Ltd 2010 : p. 8).

L'adoption de mesures d'économie d'énergie sera favorisée par une combinaison de forces du marché (hausse des prix de l'énergie, pour commencer) et de mesures publiques. Les subventions à l'achat de carburant vont à l'encontre des efforts de maîtrise de l'énergie et devraient par conséquent être évitées. Une fois mises en place des incitations privées claires en faveur des économies d'énergie, des mesures publiques d'éducation et des informations. Dans son enquête, Efficient $20(2013:$ p. 8) a conclu que «près de la moitié des exploitants agricoles et forestiers ayant répondu à l'enquête aimeraient réaliser des économies de carburant mais ignorent comment y parvenir » (p. 8). 


\subsection{L'exploitation de la fixation biologique de l'azote dans la rotation des cultures et les mélanges herbagers ${ }^{19}$}

Introduction

Les plantes cultivées de la famille des Leguminosae poussent en symbiose avec des bactéries du sol qui leur permettent de fixer l'azote contenu dans l'atmosphère et de l'utiliser à la place de l'azote des engrais de synthèse ou du fumier. Les légumineuses, qui peuvent être des graines ou des fourrages, sont capables de fixer $300 \mathrm{~kg} \mathrm{~N} / \mathrm{ha} / \mathrm{an}$ supplémentaires, ce qui rend l'apport en azote comparable aux quantités d'azote organique ou de synthèse épandues sur de nombreuses cultures. Les légumineuses peuvent atténuer les émissions de GES de quatre façons : a) par réduction des émissions directes dues aux engrais azotés ; b) par réduction des émissions de $\mathrm{CO}_{2}$ e résultant de la production d'engrais ; c) par réduction du lessivage de l'azote et des émissions indirectes de $\mathrm{N}_{2} \mathrm{O}$ qui lui sont liées ; d) par réduction de la quantité d'azote à épandre sur le suivant cultural.

\section{Impacts attendus sur les émissions de GES}

Le principal effet des légumineuses sur l'atténuation des émissions au niveau de l'exploitation est la réduction ou la suppression des émissions d'hémioxyde d'azote (voir tableau 22). L'introduction de légumineuses dans les rotations permet également le transfert d'azote résiduel aux suivants culturaux, diminuant leurs besoins en engrais et, par conséquent, les émissions de $\mathrm{N}_{2} \mathrm{O}$ (Buses et al., 2013).

Tableau 22. Réduction des émissions associée à l'introduction de légumineuses dans les systèmes de cultures et les pâturages

\begin{tabular}{|c|c|c|c|}
\hline Forme d'atténuation & Réduction des émissions & Source & Lieu \\
\hline $\begin{array}{l}\text { Réduction des } \\
\text { émissions directes et } \\
\text { indirectes de } \mathrm{N}_{2} \mathrm{O}\end{array}$ & $\begin{array}{l}\text { Légumineuses à graines : } 1.02 \mathrm{t} . \mathrm{CO}_{2} \mathrm{e} / \mathrm{ha} / \mathrm{an} \text {; } \\
\text { légumineuses fourragères : } 0.17 \mathrm{t} . \mathrm{CO}_{2} \mathrm{e} / \mathrm{ha} / \mathrm{an}\end{array}$ & Pellerin et al. (2013) & France \\
\hline $\begin{array}{l}\text { Réduction de la } \\
\text { production d'engrais }\end{array}$ & $\begin{array}{l}\text { Légumineuses à graines : } 0.95 \mathrm{t} . \mathrm{CO}_{2} \mathrm{e} / \mathrm{ha} / \mathrm{an} \\
\text { Légumineuses fourragères : } 0.16 \mathrm{t} . \mathrm{CO}_{2} \mathrm{e} / \mathrm{ha} / \mathrm{an}\end{array}$ & Pellerin et al. (2013) & France \\
\hline $\begin{array}{l}\text { Réduction totale des } \\
\text { GES* }\end{array}$ & $\begin{array}{l}\text { Légumineuses à graines : } 1.99 \mathrm{t} . \mathrm{CO}_{2} \mathrm{e} / \mathrm{ha} / \mathrm{an} \\
\text { Légumineuses fourragères : } 0,33 \mathrm{t} . \mathrm{CO}_{2} / \mathrm{ha} / \mathrm{an}\end{array}$ & Pellerin et al. (2013) & France \\
\hline $\begin{array}{l}\text { Réduction des } \\
\text { émissions directes et } \\
\text { indirectes de } \mathrm{N}_{2} \mathrm{O}\end{array}$ & Trèfle : 0.5 t. $\mathrm{CO}_{2} \mathrm{e} / \mathrm{ha} / \mathrm{an}$ & Moran et al. (2008 : p. 43) & $\begin{array}{l}\text { Royaume- } \\
\text { Uni }\end{array}$ \\
\hline $\begin{array}{l}\text { Réduction des } \\
\text { émissions de GES au } \\
\text { niveau de l'exploitation }\end{array}$ & $\begin{array}{l}\text { Substitution de céréales par des légumineuses : } \\
\text { de } 1.19 \text { t. } \mathrm{CO}_{2} \mathrm{e} / \mathrm{ha} / \mathrm{an} \text { (orge) à } \\
2.20 \text { t. } \mathrm{CO}_{2} \mathrm{e} / \mathrm{ha} / \mathrm{an} \text { (maïs) }\end{array}$ & Dequiedt et Moran (2015) & France \\
\hline
\end{tabular}

* y compris des variations mineures non précisées dans le tableau.

\section{Éléments des coûts et rapport coût-efficacité}

Aucun coût ponctuel n'est prévu, mais la substitution de cultures céréalières par des légumineuses entraînerait des coûts récurrents pour les exploitations, qui verraient fondre leurs marges brutes. La baisse des achats d'engrais de synthèse et la suppression d'opérations d'épandage peuvent être sources d'économies (voir tableau 23).

19. Cette étude de cas s'appuie en partie sur les travaux de Frelih-Larsen et al. (2014) 
Tableau 23. Coûts ou économies avec la fixation biologique de l'azote

\begin{tabular}{|c|c|c|}
\hline Coût total & Source & Lieu \\
\hline $\begin{array}{l}\text { Légumineuses à graines } 19 \mathrm{EUR} / \mathrm{ha} / \mathrm{an} \\
\text { Légumineuses fourragères ( } 31 \mathrm{EUR}) / \mathrm{ha} / \mathrm{an} \\
\text { Légumineuses à graines de } 18 \mathrm{EUR} \text { à } 19 \mathrm{EUR} / \mathrm{t} . \mathrm{CO}_{2} \\
\text { Légumineuses fourragères de (189 EUR) à (169 EUR)/t. } \mathrm{CO}_{2}\end{array}$ & Pellerin et al. (2013) & France \\
\hline $\begin{array}{l}\text { Pour le trèfle : baisse du rendement de } 30 \% \text {; baisse des achats de } \mathrm{N} \\
\text { de } 60 \% \text {; baisse des coûts de la main-d'œurre et des machines de } 5 \% \\
\text { Rapport coût-efficacité global : de } 20 \text { EUR à } 55 \text { EUR/t. } \mathrm{CO}_{2} \mathrm{e}\end{array}$ & Moran et al. (2008, p 47) & $\begin{array}{l}\text { Royaume- } \\
\text { Uni }\end{array}$ \\
\hline $\begin{array}{l}\text { Grandes cultures : «cette mesure serait peu coûteuse. » } \\
\text { Pâturages : «le coût de cette mesure pourrait être nul, voire négatif. » }\end{array}$ & Domingo et al. (2014, p 47) & UE \\
\hline $\begin{array}{l}\text { Valeurs diverses en fonction de la région et des hypothèses, moyenne : } \\
43 \mathrm{EUR} / \mathrm{t} . \mathrm{CO}_{2} \mathrm{e}\end{array}$ & Dequiedt et Moran (2015) & France \\
\hline
\end{tabular}

La variation du rapport coût-efficacité dépendra probablement du montant des recettes et du taux de rendement sacrifiés, qui subiront eux-mêmes l'influence de paramètres économiques (prix des produits végétaux et des intrants) et biophysiques (variété, climat, sols et agronomie). Cette opération risque de ne pas être rentable dans les régions où le climat n'est pas favorable.

Les réductions (et le rapport coût-efficacité) des émissions issues de la baisse de la fabrication d'engrais dépendent du type d'engrais et de son lieu de production. Vellinga et al. (2013, p. 67) analysent tout l'éventail des émissions de GES dues aux engrais azotés les plus courants (de la fabrication à la destruction).

\section{Autres effets}

Des avantages connexes peuvent être produits, notamment : a) amélioration de la qualité de l'eau par atténuation du lessivage du nitrate (Nemecek et al. 2008 ; Moran et al. 2008, p142) ; b) baisse de la demande de protéagineux de la part des pays de l'OCDE et, par conséquent, diminution du changement d'affectation des terres dans certaines régions exportatrices de protéagineux ; c) meilleure adaptation au changement climatique (en cas d'utilisation comme culture de couverture), du fait du recul de l'érosion des sols et du renforcement de leur capacité de rétention de l'eau; d) disparition des mauvaises herbes et des maladies (en cas d'utilisation comme tête d'assolement dans le cadre de la rotation des cultures); e) bénéfices éventuels pour la biodiversité (Rees et al., 2014 ; Bues et al., 2013). Les coûts connexes concernent l'accroissement de la variabilité des rendements en fonction de la variabilité des conditions météorologiques, et le déplacement éventuel de la production si les rendements faiblissent.

\section{Difficultés de mise en ouvre}

La culture des légumineuses est applicable dans de nombreux cas, sur les différents types de sols entrant en jeu dans la rotation des cultures et des pâturages; néanmoins, elle convient mieux aux sols légers à $\mathrm{pH}$ et teneur en phosphore modérés à élevés. Les résidus des cultures de légumineuses pouvant entraîner des pertes rapides d'azote, il importe de veiller à ce que le sol ne soit pas laissé en jachère après l'introduction de ces résidus. 


\section{Des mesures d'atténuation aux stratégies d'atténuation possibles : débat préliminaire}

Du point de vue de l'action des pouvoirs publics, il est difficile de tirer des messages clairs des travaux publiés sur les pratiques liées à l'atténuation des émissions, compte tenu des différences entre leurs champs d'étude, leurs méthodes et leurs hypothèses de départ, qui rendent toute comparaison entre les études problématique. Le tableau 24 reprend quelques exemples des différences qui peuvent exercer un impact non négligeable sur l'estimation du rapport coût-efficacité des diverses études. Les travaux comparant plusieurs mesures d'un pays particulier avec la même méthode sont plus à même de fournir des conclusions significatives, comme le montre l'encadré 6 sur le cas de la France.

Tableau 24. Source possible d'incommensurabilité des études

\begin{tabular}{l|l}
\hline Champ couvert & $\begin{array}{l}\text { Où sont fixées les limites du système ? } \\
\text { Quelles catégories d'émissions se trouvent dans les limites du système ? } \\
\text { Quels coûts et quels avantages sont inclus (dépenses uniquement, dépenses plus manque à gagner, coût } \\
\text { d'opportunité, etc.) ? } \\
\text { Méthode et } \\
\text { hypothèses }\end{array}$ \\
$\begin{array}{l}\text { Quelle méthode est employée pour déterminer la réduction des émissions, par exemple, quels facteurs } \\
\text { Comment les émissions issues du changement d'affectation des terres sont-elles calculées et réparties ? } \\
\text { Quelles hypothèses sont établies à propos de la séquestration du carbone dans le sol ? } \\
\text { Quels taux d'actualisation sont utilisés ? } \\
\text { Comment l'utilisation en présence de politiques inchangées et de mesures alternatives a-t-elle été estimée } \\
?\end{array}$ \\
\hline
\end{tabular}

Les résultats d'un sous-ensemble distinct de mesures d'atténuation couramment étudiées dans la documentation internationale analysée sont comparés dans le graphique 7, afin de montrer les variations possibles du rapport coût-efficacitée ${ }^{20}$. Ces études ont été jugées comparables dans leurs grandes lignes, parce qu'elles utilisent des méthodes similaires d'estimation ascendante des coûts, qu'elles comportent des données sur les coûts (et non simplement des examens) et qu'elles sont suffisamment détaillées pour permettre des comparaisons. Les résultats sont présentés pour les mesures le plus fréquemment étudiées, à savoir, celles qui sont traitées dans cinq études au moins. Toutefois, un examen plus approfondi a révélé que les résultats des données sur le rapport coût efficacité n'étaient pas toujours suffisamment transparents. C'est pourquoi certaines mesures sont représentées sur le diagramme alors que le nombre d'estimations de leur rapport coût-efficacité est inférieur à cinq.

Le graphique 7 montre que le rapport coût-efficacité d'une mesure peut varier fortement d'une étude à l'autre, même lorsque les approches adoptées sont sensiblement identiques. Au sein même des études, les rapports coût-efficacité peuvent présenter des écarts importants pour certaines mesures, telles que l'optimisation de l'épandage d'engrais azotés, mais ces différences montrent simplement, en général, que les champs couverts par les études ne sont pas identiques (à savoir, qu'ils varient en fonction de la délimitation du système ou des catégories de coûts et d'émissions incluses dans les limites de ces systèmes).

20. Ces mesures ne correspondent pas exactement à celles présentées à la section 4 . La section 4 traite d'une gamme plus étendues de pratiques, associées à des méthodes et des données ne permettant pas toutes la comparaison. 


\section{Encadré 6. Potentiel d'atténuation et coût de dix actions techniques le cas de la France}

Au nom de l'Agence de l'environnement et de la maîtrise de l'énergie (ADEME), du ministère de l'Agriculture, de l'agroalimentaire et de la forêt (MAAF) et du ministère de l'Écologie, du développement durable et de l'énergie (MEDDE), I'Institut national de la recherche agronomique (INRA) a réalisé une étude sur le potentiel d'atténuation et le coût de dix actions techniques visant à réduire les émissions de gaz à effet de serre de l'agriculture en France (Pellerin et al., 2013).

Les mesures retenues correspondent à quatre leviers techniques : i) diminuer l'épandage d'engrais azoté minéral ; ii) stocker le carbone dans le sol et dans la biomasse ; iii) modifier l'alimentation du bétail ; iv) recycler le fumier pour produire de l'énergie et diminuer la consommation de combustible fossile. Différents critères ont été utilisés afin de sélectionner les mesures pour l'étude, notamment le fait que l'adoption de la mesure ne devrait pas réduire la production de plus de $10 \%$, la nécessité pour la mesure de cibler les pratiques agricoles et non l'amont ou l'aval de la filière d'approvisionnement, et la disponibilité actuelle de la technologie (concernée par la mesure). Le potentiel annuel de réduction des émissions de GES de chaque mesure repose sur des estimations du coût et du potentiel de réduction par unité, sur l'applicabilité (en tenant compte de la superficie des terres et du nombre d'animaux) et sur la projection de l'adoption des mesures entre 2010 et 2030.

Les résultats de l'étude montrent que plusieurs mesures ont un coût négatif, notamment la gestion des pâturages (accroissement de la part des légumineuses dans les prairies temporaires et allongement de la durée de vie de ces dernières, par exemple) et la baisse de la consommation de combustible fossile (par le renforcement de l'isolation et des dispositifs de chauffage des stabulations, ainsi que par l'optimisation de la consommation de gazole des tracteurs par le réglage de leurs moteurs et l'adoption de l'écoconduite). La gestion des engrais par l'ajustement des taux et du calendrier d'épandage, ainsi que l'amélioration de l'utilisation et de l'épandage du fumier, représentent également des mesures dont les coûts sont légèrement négatifs et le potentiel de réduction des émissions élevé.

La réduction ou la suppression du labour ainsi que la méthanisation du fumier du bétail sont des mesures de réduction à coût modéré (moins de $25 \mathrm{EUR} / \mathrm{CO}_{\mathrm{e}}$ ). Les mesures à coût élevé (supérieur à $25 \mathrm{EUR} / \mathrm{CO}_{2} \mathrm{e}$ ) comprennent les mesures relatives à l'utilisation des terres et à leur couverture, notamment par des haies, des bandes enherbées et des cultures de couverture. Dans l'ensemble, les résultats de l'étude démontrent que la plus grande partie des mesures présentant un potentiel de réduction des émissions à coût négatif est liée à la gestion de l'azote et que leur valeur augmente lorsque les émissions induites (diminution de la fabrication d'engrais azotés de synthèse) et les bénéfices parallèles pour l'environnement (amélioration de la qualité de l'eau et de l'air) sont également pris en compte.

Source: Pellerin et al. (2013), "Quelle contribution de l'agriculture française à la réduction des émissions de gaz à effet de serre ? Potentiel d'atténuation et coût de dix actions techniques : le cas de la France ", INRA, France, 92 pages.

Diverses stratégies peuvent être envisagées pour encourager l'adoption de mesures d'atténuation des émissions de GES, allant de l'éducation à la réglementation en passant par les mesures économiques d'incitation - comme le montre le tableau 25 - et dont la pertinence dépendra du coût des mesures d'atténuation pour la société. Plusieurs mesures d'atténuation représentent un coût net pour les acteurs privés - c'est-à-dire que les coûts pour l'agriculteur sont supérieurs aux bénéfices qu'il en retire - mais un bénéfice net pour la société — les bénéfices totaux pour l'agriculteur et pour la société, progrès pour l'environnement compris, sons supérieurs aux coûts totaux. Une stratégie publique peut être nécessaire pour que ces mesures bénéfiques pour la société soient adoptées. Pannell (2008) élabore un cadre général fondé sur les bénéfices nets privés et publics de l'adoption de pratiques favorables à l'environnement et décrit le mécanisme qui devrait être retenu afin d'optimiser le profit net d'une intervention de l'action publique, si tant est qu'il en existe un. 
Graphique 7. Estimations minimales (L) et maximales (H) du rapport coût-efficacité (EUR/t. CO2e) des mesures d'atténuation envisagées dans 12 études de MACC*

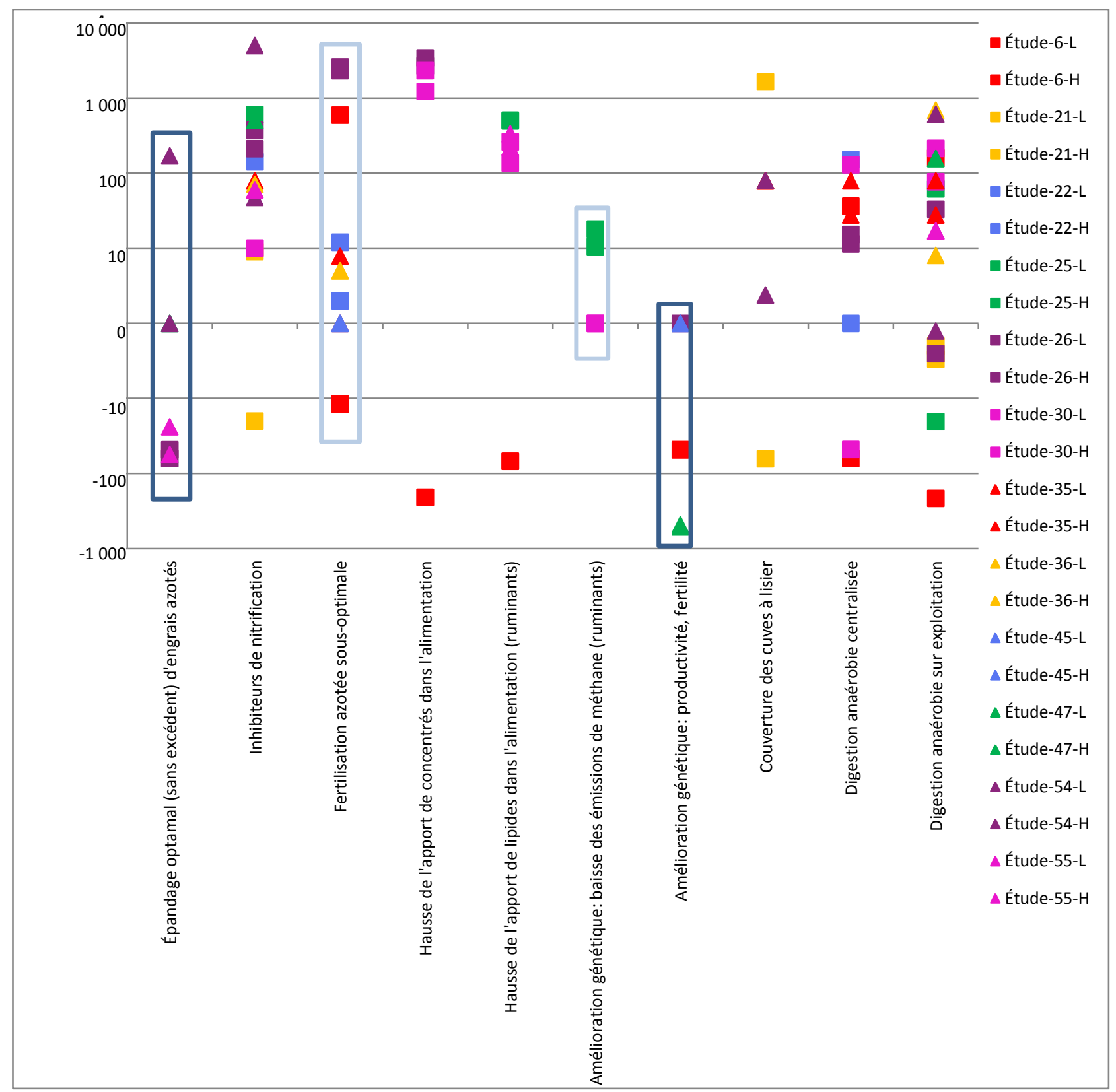

Notes: axe des y construit selon une échelle logarithmique ; $N$ : azote ; $A D$ : digestion anaérobie Voir tableau 27 pour les références des études. 
Tableau 25. Exemples de stratégies d'atténuation des GES

\begin{tabular}{|c|c|c|}
\hline Stratégie & Types d'intervention & Exemples de stratégies d'atténuation \\
\hline $\begin{array}{l}\text { Information et } \\
\text { éducation }\end{array}$ & $\begin{array}{l}\text { - Services de conseil et d'information } \\
\text { fournis par le gouvernement ; } \\
\text { - Étiquetage; } \\
\text { - Rapportage et divulgation ; } \\
\text { - Stigmatisation. }\end{array}$ & $\begin{array}{l}\text { Gains de productivité à travers l'investissement public } \\
\text { dans la R-agricole aux États-Unis (voir Jones et al., 2013). } \\
\text { Délivrance de conseils à travers l'initiative « Farming for a } \\
\text { better climate » (Gouvernement écossais 2013b : p. 147). }\end{array}$ \\
\hline Autoréglementation & $\begin{array}{l}\text { - Codes de bonne pratique } \\
\text { - Co-réglementation }\end{array}$ & - Woodland Carbon Code (Forestry Commission, 2014). \\
\hline Méthodes incitatives & $\begin{array}{l}\text { - Création de marchés; } \\
\text { - Droits/quotas échangeables ; } \\
\text { - Plafonnement des prix ; prix } \\
\text { d'objectif ; } \\
\text { - Taxes; } \\
\text { - Primes : crédits d'impôt, subventions, } \\
\text { etc. }\end{array}$ & $\begin{array}{l}\text { Versement de subventions à travers le programme } \\
\text { agriculture et développement rural de l'UE afin de rétablir } \\
\text { les tourbières } \\
\text { Aux États-Unis, versement de subventions à travers le } \\
\text { Conservation Reserve Program afin de déclasser des } \\
\text { terres labourables (voir Jones et al., 2013). }\end{array}$ \\
\hline $\begin{array}{l}\text { Réglementation } \\
\text { classique et ses } \\
\text { variantes }\end{array}$ & $\begin{array}{l}\text { Couvre un large éventail d'instruments } \\
\text { officiels, souvent étayés de sanctions, } \\
\text { notamment interdiction d'activités, } \\
\text { autorisations préalables, normes, etc. }\end{array}$ & $\begin{array}{l}\text { Directive sur les nitrates de l'UE - fixe les taux et le } \\
\text { calendrier de l'épandage des nutriments. }\end{array}$ \\
\hline
\end{tabular}

Source : repris de MacLeod et al. (2009) : p. 11

Du point de vue du coût global pour les acteurs privés, les mesures du graphique 7 se répartissent en trois groupes :

a) les mesures qui, selon un large accord (voire un consensus), ont un faible coût ou un coût négatif et dont l'adoption devrait pouvoir être encouragée par des stratégies axées sur l'éducation et l'information : l'optimisation de l'épandage des engrais azotés; la réduction des émissions de $\mathrm{CH}_{4}$ issues de la fermentation entérique par amélioration génétique axée sur la productivité/fertilité (encadrées d'un trait foncé sur le graphique 7). Le cas de l'amélioration de l'efficacité énergétique des engins agricoles, non représenté sur le graphique en raison d'un nombre insuffisant d'estimations, peut également entrer dans cette catégorie ;

b) les mesures susceptibles d'avoir un coût positif modéré, mais qui apporteraient un bénéfice net à la société (le coût de leur mise en œuvre étant inférieur au coût des émissions pour la société) et pour lesquelles une stratégie fondée sur des mesures d'incitation pourrait être justifiée : la réduction de l'épandage d'azote à un niveau inférieur au niveau (financièrement) optimal; la diminution spécifique des émissions de $\mathrm{CH}_{4}$ issues de la fermentation entérique par amélioration génétique (encadrées d'un trait clair sur le graphique 7);

c) les autres mesures, de rapports coût-efficacité très divers et capables d'atténuer efficacement les émissions à condition d'être ciblées précisément pour éviter tout coût superflu.

Les deux dernières catégories conduisent à se demander comment recourir aux incitations pour promouvoir l'objectif d'atténuation des émissions à moindre coût. Une stratégie possible consisterait à établir des signaux-prix généraux (taxes ou marché des droits d'émissions) afin de renforcer les incitations à l'adoption des mesures d'atténuation par les agriculteurs lorsqu'elles sont pertinentes. Ces signaux-prix encourageraient également l'innovation et la mise au point de technologies et pratiques innovantes d'atténuation des émissions en agriculture. Une autre stratégie pourrait être l'application de politiques d'incitation ciblées visant à soutenir quelques-unes des mesures techniques les plus prometteuses au niveau local, notamment en faisant appel à des paiements volontaires (comme dans le cadre de la Politique agricole commune de l'UE).

La première catégorie présente sans doute des avantages du fait qu'elle ne demande pas de connaissance des spécificités locales. Néanmoins, elle risque de poser des difficultés de mise en œuvre et de suivi. Seuls quelques pays ont adopté un système de taxes sur le carbone et, jusqu'à présent, 
l'agriculture reste exclue dans une large mesure du champ d'application de ces politiques. En NouvelleZélande et en République de Corée, des régimes d'échange de droits d'émission ont été introduits récemment et prévoient de s'appliquer à l'agriculture. Le secteur agricole néo-zélandais devait être pleinement pris en compte dans le régime national en 2015, mais la mise en œuvre a été retardée (OCDE, 2011 ; Banque mondiale, 2014 : p. 64). En République de Corée, le régime de 2015 inclut explicitement l'agriculture (EDF et IETA, 2013), mais il est trop tôt pour évaluer sa mise en œuvre. Aucun des 18 autres systèmes de tarification du carbone prévus ou en vigueur fin 2014 ne concernaient explicitement l'agriculture (Banque mondiale, 2014). Entre autres facteurs, la création de marchés est difficile lorsque les sources d'émissions sont nombreuses, comme dans l'agriculture. Le nombre de sources d'émissions pose également des problèmes de suivi et de contrôle de l'application de certaines mesures, notamment celles associées à l'amélioration de l'efficience de l'utilisation de l'azote. Enfin, le potentiel de l'agriculture en termes de compensation carbone volontaire a également été analysé mais a rarement été exploité.

Par ailleurs, l'adoption de mesures ciblées à l'appui de l'atténuation peut se heurter à toutes sortes de difficultés au niveau de la mise en œuvre. Premièrement, la question de la combinaison des mesures peut s'avérer problématique. Une mesure d'atténuation peut s'appliquer seule ou conjointement avec d'autres. En cas d'application conjointe, des interférences peuvent se produire, ce qui signifie que le taux de réduction des émissions et le rapport coût-efficacité des mesures peuvent être assez différents si elles sont prises séparément ou en conjonction (voir MacLeod et al., 2010a, Hristov et al., 2013 : p. 121 ; Sikirica et al., 2014 : p. 24). Si, par exemple, une exploitation ensemence ses terres de légumineuses et restreint l'épandage d'engrais azotés, le potentiel de réduction des émissions par amélioration de l'efficience de l'épandage d'azote diminuera et les mesures axées sur l'efficience de l'azote seront moins rentables. À l'inverse, l'optimisation du taux hydrique des sols par amélioration du drainage peut renforcer l'effet des inhibiteurs de nitrification sur l'atténuation ou faciliter l'étalement des épandages de nutriments. Par conséquent, il est primordial que toute mesure ciblée tienne compte de ces synergies.

Deuxièmement, il peut être également nécessaire de tenir compte de l'incidence du déplacement de la production. Certaines mesures d'atténuation peuvent modifier en profondeur les taux de production (les inhibiteurs de nitrification peuvent améliorer les rendements par hectare, alors que le boisement restreint la production vivrière). Compte tenu des prévisions de croissance de la demande alimentaire, une baisse de la production en un point donné débouchera sur un déplacement de la production (et des émissions qui lui sont associées) vers d'autres régions ou pays. Afin de tenir compte de ce phénomène, les analyses du rapport coût-efficacité des mesures d'atténuation devraient, en principe, comprendre le calcul de la réduction des émissions nette de tout déplacement, mais un tel calcul peut être complexe à mettre en pratique et est rarement réalisé.

Troisièmement, la variabilité du rapport coût-efficacité peut également être problématique pour la conception de stratégies, en particulier si elles doivent être ciblées. Les éléments constitutifs du rapport coût-efficacité, notamment les coûts et le taux de réduction des émissions, présentent souvent de fortes variations dans l'espace, dans le temps et en fonction du type d'exploitation. Par exemple, compte tenu de la variation des prix des engrais et de la production au fil des ans et entre les pays, le coût de la généralisation de la culture du maïs aux dépens des prairies destinées à l'alimentation du bétail peut être très différent d'un agriculteur à un autre. Le ciblage de l'épandage d'engrais azotés pour réduire la pollution est un autre exemple, l'incidence sur les émissions de GES pouvant varier en fonction des zones climatiques, des types de sols et des conditions météorologiques. La plupart des études du rapport coûtefficacité décrivent la situation moyenne présumée sans tenir compte de cette variabilité. De ce fait leurs résultats doivent davantage jouer un rôle d'orientation que de prescription applicable aux exploitations prises séparément ou sur des années isolées. À l'avenir, les travaux pourraient porter sur les différents potentiels et coûts d'atténuation des émissions pour, par exemple, différents types et différentes tailles d'exploitations agricoles. Les différences de coûts et d'applicabilité des mesures en fonction de l'environnement socio-économique (institutions, politiques, résilience du secteur) devraient également entrer en ligne de compte, même si souvent, tel est déjà le cas, dans une certaine mesure, dans les études du rapport coût-efficacité à l'échelle mondiale ou régionale. 
Quatrièmement, toutes les données et les hypothèses sur lesquelles reposent les études comportent des incertitudes qui se répercutent sur les estimations, en particulier en ce qui concerne les taux de réduction des émissions et l'estimation du taux d'adoption future des mesures (Eory et al., à paraître). Ces incertitudes sont rarement quantifiées et présentées dans les analyses. Dans l'idéal, les études des rapports coût-efficacité devraient s'accompagner d'une analyse des incertitudes afin de fournir aux autorités des informations sur la fourchette probable du potentiel et des coûts de l'atténuation des émissions. La complexité des modèles utilisés dans les études ne s'y prêtant pas toujours, une autre solution consiste à effectuer une analyse de sensibilité des hypothèses qui seront probablement les plus importantes.

Enfin, la non adoption de mesures d'atténuation présumées doublement gagnantes ou à coût négatif mérite peut-être d'être examinée plus en détail. Les mesures qui, selon les méthodes d'estimation ascendante, ont un coût négatif sont parfois désignées comme «doublement gagnantes », car elles permettent à la fois de réduire les émissions et de réaliser des économies. En théorie, ces mesures devraient être largement adoptées, mais la réalité est souvent autre. Outre les erreurs de calcul et les limites des méthodes d'estimation, ce constat donne lieu à un intéressant "paradoxe », en apparence. Ce paradoxe peut s'expliquer par le fait qu'une mesure risque d'être plus coûteuse dans la pratique que l'étude ne le suggère. Certains coûts importants, tels que les coûts de transaction et de l'apprentissage liés à l'adoption des mesures, sont difficiles à quantifier et sont de ce fait rarement pris en compte dans le calcul du rapport coût-efficacité. L'aversion aux risques des agriculteurs peut également les décourager d'adopter des pratiques particulières. Par ailleurs, il a été avancé que les agriculteurs n'adoptent pas nécessairement les mesures doublement gagnantes car leurs choix ne sont pas guidés par l'optimisation rationnelle du profit. Leur prise de décision obéit davantage à des facteurs internes (connaissance, habitudes et attitudes vis-à-vis du risque), des facteurs sociaux (normes et rôles), le cadre d'action et d'autres contraintes auxquelles les exploitations agricoles sont soumises (Pike, 2008). Moran et al. (2013) sont même allés jusqu'à suggérer qu'il conviendrait de remplacer les explications génériques du doublement gagnant, qui constituent une base pratique d'un point de vue politique mais excessivement simpliste et potentiellement contre-productive pour établir une stratégie d'atténuation, par une connaissance des caractéristiques psychologiques et évolutives.

\section{Principaux enseignements : quelques mesures notables, beaucoup d'autres de rapport coût- efficacité variable et travaux de recherche nécessaires sur les estimations et les solutions possibles à l'appui de l'application}

Déjà nombreux, les travaux sur le rapport coût-efficacité des mesures d'atténuation des émissions de GES agricoles se multiplient, preuve qu'elles peuvent susciter l'intérêt et offrir des possibilités. Toutefois, l'éventail de mesures et d'études est si large qu'il est difficile d'en tirer des enseignements. Les études du rapport coût-efficacité doivent nécessairement traduire la complexité des processus biophysiques et économiques par des résultats qui puissent être communiqués aux responsables publics.

Les différences existant entre les méthodes employées dans les études obligent à faire preuve de prudence pour dresser des comparaisons et tirer des conclusions. Malgré cette réserve, la présente analyse montre que certaines mesures, notamment celles reposant sur le renforcement de l'efficience de l'utilisation des engrais et sur l'amélioration génétique du bétail - voire, sur les progrès de l'efficacité énergétique des engins agricoles -, sont régulièrement considérées comme fournissant des possibilités d'atténuation très avantageuses.

La variation du rapport coût-efficacité dans les diverses options techniques et entre elles est un thème récurrent dans les études analysées. De nombreux facteurs expliquent cette variation, mais le principal enseignement est que pour exploiter au mieux les opportunités et optimiser l'efficience des politiques d'atténuation, les mesures doivent être adaptées aux lieux et aux types d'exploitations.

Il reste à déterminer s'il est préférable d'encourager l'adoption des mesures par des programmes ciblés ou par des stratégies plus générales d'incitation économique. Les deux principales options sont ici l'utilisation d'instruments économiques sectoriels ou plus généraux (taxe ou plafonnement et échange, par exemple) ou l'introduction de méthodes liées à des paiements volontaires pour promouvoir des mesures 
ciblées. La première option peut encourager l'adoption d'une gamme de mesures fondées sur les contraintes des agriculteurs, mais les instruments sur lesquels elles reposent peuvent être difficile à introduire et ne sont pas utilisés actuellement dans l'agriculture. La deuxième option pose d'autres problèmes liés à la conception et à la mise en œuvre d'une stratégie, notamment l'hétérogénéité spatiale des coûts et de l'efficacité des pratiques d'atténuation ainsi que la manière d'adapter le soutien en présence d'une telle hétérogénéité. En particulier, les pouvoirs publics devraient être capables de : a) prévoir le rapport coût-efficacité des différentes combinaisons de mesures; b) tenir compte de l'incidence des déplacements ; c) comprendre pourquoi les agriculteurs sont parfois réticents à adopter des mesures d'atténuation «doublement gagnantes » en apparence et adapter les informations et orientations fournies en conséquence. Enfin, il convient de remarquer que la mise en œuvre des mesures d'atténuation peut avoir une incidence positive ou négative imprévue sur, par exemple, la qualité de l'air, la qualité de l'eau, la qualité du sol, la biodiversité et la santé animale. La prise en compte de ces effets dans l'analyse pourrait modifier sensiblement le rapport coût-efficacité relatif des mesures d'atténuation des GES.

Le travail d'analyse doit être poursuivi pour apporter des réponses à ces problèmes et trouver comment inscrire les mesures d'atténuation dans le cadre plus vaste des politiques climatiques, agricoles et environnementales. Deux projets de l'OCDE s'intéresseront prochainement aux synergies et aux interactions entre la productivité agricole, l'atténuation des GES et l'adaptation au changement climatique ; les obstacles à l'adoption des pratiques agricoles susceptibles d'être doublement gagnantes et plus favorables au climat seront également évalués dans ce cadre. 


\section{Références}

AEA Technologies (2010), «Energy Marginal Abatement Cost Curve for English Agricultural Sector », Rapport $\mathrm{n}^{\circ} \mathrm{EC} 0103$ du ministère de l'Environnement, de l'alimentation et des affaires rurales, Londres, Royaume-Uni.

AgriClimateChange (2013), "Climate Friendly Agriculture: Evaluations and improvements for energy and greenhouse gas emissions at the farm level in the European Union", Solagro, Toulouse. www.agriclimatechange.eu

Akiyama, H., X. Yan et K. Yagi (2010), "Evaluation of Effectiveness of Enhanced-Efficiency Fertilizers as Mitigation Options for N2O and NO Emissions from Agricultural Soils: Meta-Analysis", Global Change Biology, Vol.16, pp. 1837-1846.

Alexandratos, N. et J. Bruinsma (2012), World agriculture towards 2030/2050: the 2012 revision. Document de travail ESA no 12-03. Rome, FAO.

Amann, M. et al. (2008), "Emission scenarios for non-CO2 greenhouse gases in the EU-27, Mitigation potentials and costs in 2020, Final Report", International Institute of Applied Systems Analysis (IIASA) Laxenbourg, Autriche

Amann, M. et al. (2011), "Cost-effective control of air quality and greenhouse gases in Europe: Modeling and policy applications”, Environmental Modelling and Software, Vol. 26, pp. 1489-1501.

Anthony, S. et al. (2008) Quantitative assessment of scenarios for managing trade-off between economics, environment and media, rapport $\mathrm{n}^{\mathrm{o}} \mathrm{WQ} 0106 \mathrm{du}$ ministère britannique de l'Environnement, de l'alimentation et des affaires rurales (Module 6), ministère britannique de l'Environnement, de l'alimentation et des affaires rurales, ADAS, North Wyke Research, Royaume-Uni.

Bajželj,B. et al. (2014), "Importance of food-demand management for climate mitigation", Nature Climate Change, Vol. 4,pp. 924-929.

Ball, B.C., I.P. McTaggart et A. Scott, A. (2004), "Mitigation of greenhouse gas emissions from soil under silage production by use of organic manures or slow-release fertilizer", Soil Use and Management, Vol. 20, pp. 287-295.

Banque mondiale (2014), « State and Trends of Carbon Pricing 2014 », Banque mondiale, Washington, DC, États-Unis. doi : 10.1596/978-1-4648-0268-3.

Bates, J. (2001), "Economic Evaluation of Emission Reductions of Nitrous Oxides and Methane in Agriculture in the EU - Bottom-up Analysis", Ecofys Energy and Environment, AEA Technology Environment et Université technique nationale d'Athènes.

Bates, J. et al. (2009), "Agriculture: methane and nitrous oxide”, in Sectoral Emission Reduction Potentials and Economic Costs for Climate Change (SERPEC-CC), Ecofys, AEA Energy and Environment, Didcot, Royaume-Uni.

Beach, R. et al. (2008), "Mitigation potential and costs for global agricultural greenhouse gas emissions", Agricultural Economics, Vol. 38, pp. 109-115.

Beauchemin, K. et al.(2008), "Nutritional management for enteric methane abatement: a review", Australian Journal of Experimental Agriculture, Vol. 48, pp.21-27.

Bongiovanni, R. et J. Lowenberg-Deboer (2004), "Precision Agriculture and Sustainability", Precision Agriculture, Vol. 5, pp.359-387. 
Bosello, F. et al. (2005)," Cost efficiency and effectiveness of GHG mitigation policies and measures in the agroforestry sector: a survey of the economic literature", MEACAP WP2 D6, Fondazione Eni Enrico Mattei (FEEM), Venice.

Boyer, C. et al. (2011), "Profitability of variable rate nitrogen application in wheat production", Precision Agriculture, Vol.12, pp. 473-487.

Brink, C. et al. (2005), "Cost-effective emission abatement in agriculture in the presence of interrelations: cases for the Netherlands and Europe", Ecological Economics, Vol. 53(1), pp.59-74.

Bues, A. et al. (2013), "The environmental role of protein crops in the new common agricultural policy", Parlement européen. http://www.europarl.europa.eu/delegations/en/studiesdownload.html?languageDocument=EN\&file=933 $\underline{70}$

Cameron K.C. et al. (2002), "Nutrient and pesticide transfer from agricultural soils to water in New Zealand", In Haygarth P and S. Jarvis (eds.), Agriculture, Hydrology and Water Quality, pp. 373-393, CAB International, Wallingford, Royaume-Uni.

Carbon Trust (2012), “Agriculture and horticulture: Introducing energy saving opportunities for farmers and growers", Londres : The Carbon Trust, Royaume-Uni. http://www.carbontrust.com/media/39180/ctv009_agriculture_and horticulture.pdf

Cayuela, M. L. et al. (2014), "Biochar's role in mitigating soil nitrous oxide emissions: A review and metaanalysis", Agriculture, Ecosystems \& Environment, Vol. 191, pp. 5-16.

Centre commun de recherche (2013), « Best Available Techniques (BAT) Reference Document for the Intensive Rearing of Poultry and Pigs Industrial, directive 2010/75/EU relative aux émissions industrielles (prévention et réduction intégrées de la pollution) version 2 »- août 2013, Séville : bureau européen du GIEC, http://eippcb.jrc.ec.europa.eu/reference/

Coderoni, S. et al. (2015), "Climate change mitigation options in the Italian livestock sector", EuroChoices 14(1): 17-24.

Collier, U. et al. (2013), Reducing the UK's carbon footprint and managing competitiveness risks, Committee on Climate Change, Londres. http://www.theccc.org.uk/wp-content/uploads/2013/04/CF-CSummary-Rep-web1.pdf

Cornet, M., J. et al. (2013), Scénarios pour une Belgique bas carbone à l'horizon 2050. Service public fédéral Santé publique, sécurité de la chaîne alimentaire et environnement, Belgique. http://www.klimaat.be/2050/files/2513/8625/2687/Low_Carbon_Scenarios_for_BE_2050__Final_Report.pdf

De Cara, S. et al. (2005), "Methane and nitrous oxide emissions from agriculture in the EU: A spatial assessment of sources and abatement costs", Environmental \& Resource Economics, Vol. 32, pp. 551583.

De Cara, S. et P.A. Jayet (2011), "Marginal abatement costs of greenhouse gas emissions from European agriculture, cost effectiveness, and the EU non-ETS burden sharing agreement", Ecological Economics, Vol. 70, pp. 1680-1690.

DeAngelo, B.J. et al. (2006), "Methane and nitrous oxide mitigation in agriculture", Energy Journal, Vol. 27, pp. 89-108.

de Bruin, K. et al.(2009), "Adapting to climate change in the Netherlands: an inventory of climate adaptation options and ranking of alternatives", Climatic Change, Vol. 95, pp. 23-45.

Dequiedt, B. et D. Moran (2015), "The cost of emission mitigation by legume crops in French agriculture", Ecological Economics, Vol. 110, pp. 51-60.

Desjardins, R. L. et al. (2001), "Canadian greenhouse gas mitigation options in agriculture”, Nutrient Cycling in Agroecosystems, Vol. 60, pp. 317-326. 
Di, H.J. et K.C. Cameron (2002), "The use of a nitrification inhibitor, dicyandiamide (DCD), to decrease nitrate leaching and nitrous oxide emissions in a simulated grazed and irrigated grassland", Soil Use and Management, Vol. 18, pp. 395-403.

Di, H.J. et K.C. Cameron (2003), "Mitigation of nitrous oxide emissions in spray-irrigated grazed grassland by treating the soil with dicyandiamide, a nitrification inhibitor", Soil Use and Management, Vol. 19, pp. 284-290.

Di, H.J. et K.C. Cameron (2004), "Effects of the nitrification inhibitor dicyandiamide on potassium, magnesium and calcium leaching in grazed grassland", Soil Use and Management, Vol. 20, pp. 2-7.

Di, H.J. et al. (2007), "Comparison of the effectiveness of a nitrification inhibitor, dicyandiamide, in reducing nitrous oxide emissions in four different soils under different climatic and management conditions", Soil Use and Management, Vol. 23, pp. 1-9.

Diekmann, F. et M.T. Batte (2010), "2010 Ohio Farming Practices Survey: Adoption and Use of Precision Farming Technology in Ohio", The Ohio State University, États-Unis

Domingo, J.E. et al. (2014), "Measures at the farm level to reduce greenhouse gas emissions from agriculture", Notes de la version provisoire. Bruxelles : Parlement européen.

Dominguez, I.P. et al. (2009), "Trading schemes for greenhouse gas emissions from European agriculture: A comparative analysis based on different implementation options", Review of Agricultural and Environmental Studies - Revue d'Etudes en Agriculture et Environnement, Vol. 90, pp. 287-308.

Domínguez, I.P. et al. (2012), "Agricultural GHG emissions in the EU: an exploratory economic assessment of mitigation policy options", Commission européenne, Centre commun de recherche, Institut pour les études de prospective technologique, Séville. http://ftp.jrc.es/EURdoc/JRC69817.pdf

Doreau, M. et al. (2014), "Mitigation of enteric methane for French cattle: Potential extent and cost of selected actions”, Animal Production Science, Vol 54,pp.1417-1422

Durandeau, S. et al. (2010), "Coupling biophysical and micro-economic models to assess the effect of mitigation measures on greenhouse gas emissions from agriculture", Climatic Change, Vol. 98, pp. 5173.

EDF (Environment Defense Fund) et IETA (International Emissions Trading Association (2013), "The World's Carbon Markets: A Case Study Guide to Emissions Trading- South Korea”, EDF et IETA, Washington,

États-Unis. http://www.ieta.org/assets/EDFCaseStudyMarch2014/south\%20korea\%20ets\%20case\%20study\%20ma rch\%202014.pdf

Efficient 20 (2012), "Fuel Efficiency: Reducing your fuel bill and helping the environment", Efficient 20, Commission Européenne, Rennes, France.

Efficient 20 (2013), “Advisor's brochure Teaching from the Efficient 20 project Tips for encouraging farmers and foresters to reduce their fuel consumption", Efficient 20, Commission Européenne, Rennes, France.

Eory, V. (2012), "Advice on the inclusion of precision farming in the RPP2 budget", ClimateXChange, Edinburgh. http://www.climatexchange.org.uk/reducing-emissions/advice-inclusion-precision-farming$\operatorname{rpp} 2 /$.

Eory, V. (2014), "Evaluating the use of marginal abatement cost curves applied to greenhouse gas abatement in the UK agriculture", thèse de doctorat, décembre 2014, SRUC/Edinburgh University, Royaume-Uni.

Eory,V. et al. (2014), “Assessing uncertainty in the cost-effectiveness of agricultural greenhouse gas mitigation”, Agricultural Economics Society 88th Annual Conference, 9-11 Avril 2014, AgroParisTech, Paris, France.

Eory,V. et al. (2013), "Multiple-pollutant cost-effectiveness of greenhouse gas mitigation measures in the UK agriculture”, Environmental Science \& Policy, Vol. 27:pp. 55-67. 
Environmental Protection Agency-Queensland, Office of Climate Change (2008), “An enhanced Queensland Marginal Abatement Cost Curve", EPA Queensland, Brisbane, Australie.

Farming for a Better Climate (2014), "Practical Guide: Optimising Tractor Fuel Use", Scottish Rural College, Edinburgh.Available at: www.sruc.ac.uk/downloads/120198/improve farm_efficiency.

Forestry Commission (2014), "Woodland Carbon Code: Requirements for voluntary carbon sequestration projects”, Forestry Commission, Londres. www.forestry.gov.uk/forestry/INFD-863FFL.

Frelih-Larsen, A. et al. (2014), "Mainstreaming climate change into rural development policy post 2013", Rapport final, Ecologic Institute, Berlin. http://bookshop.europa.eu/en/mainstreaming-climate-changeinto-rural-development-policy-post-2013pbML0614002/?CatalogCategoryID=1PKep2Ix.xIAAAEn5nxL_IUy

Genesis Faraday (2008), "A study of the scope for the application of research in animal genomics and breeding to reduce nitrogen and methane emissions from livestock based food chains", Rapport $\mathrm{n}^{\mathrm{o}} \mathrm{AC} 0204$ du ministère de l'Environnement, de l'alimentation et des affaires rurales, Royaume-Uni.

Gerber, P.J et al. (2013), « Lutter contre le changement climatique grâce à l'élevage - Une évaluation des émissions et des opportunités d'atténuation au niveau mondial », Organisation des Nations Unies pour l'alimentation et l'agriculture (FAO), Rome.

GIEC (Groupe d'experts intergouvernemental sur l'évolution du climat) (2013), «Climate Change 2013: The Physical Science Basis. Contribution of Working Group I to the Fifth Assessment Report of the Intergovernmental Panel on Climate Change », Stocker, T. F et al. (éd.), Cambridge University Press, Royaume-Uni.

GIEC (2007), "Climate Change 2007: Working Group I: The Physical Science Basis, IPCC AR 4 Report”, GIEC, Genève, Suisse. http://www.ipcc.ch/publications_and_data/ar4/wg1/en/ch2s2-10-2.html.

GIEC (2006), «Lignes directrices 2006 du GIEC pour les inventaires nationaux de gaz à effet de serre, préparé par le programme du GIEC des inventaires nationaux de gaz à effet de serre, Volume 4 : Agriculture, foresterie et autres affectations des terres », Eggleston, H. S., Buendia, L., Miwa, K., Ngara, T. et Tanabe, K., Institute for Global Environmental Strategies (IGES), Japon.

Glenk, K. et S. Colombo (2011), "Designing policies to mitigate the agricultural contribution to climate change: an assessment of soil based carbon sequestration and its ancillary effects", Climatic Change, Vol. 105, pp. 43-66.

Glenk, K. et al. (2014) "Adoption of greenhouse gas mitigation in agriculture: an analysis of dairy farmers' preferences and adoption behaviour”, Ecological Economics, Vol. 108, pp. 49-58.

Godwin, R. J. et al. (2003), “An Economic Analysis of the Potential for Precision Farming in UK Cereal Production”. Biosystems Engineering, Vol. 84, pp. 533-545.

Golub, A. et al. (2009), "The opportunity cost of land use and the global potential for greenhouse gas mitigation in agriculture and forestry”, Resource and Energy Economics, Vol. 31, pp. 299-319.

Gouvernement écossais (2013a), Scottish Greenhouse Gas Emissions 2011, Édimbourg : Gouvernement écossais, Royaume-Uni.

Gouvernement écossais (2013b), Low Carbon Scotland: Meeting our Emissions Reduction Targets 20132027, The Draft Second Report on Proposals and Policies, Édimbourg: Gouvernement écossais, Royaume-Uni.

Gouvernement néo-zélandais (2014) New Zealand's Greenhouse Gas Inventory 1990-2012, Wellington : Gouvernement néo-zélandais, Nouvelle-Zélande.

Grainger, C. et K.A. Beauchemin (2011), "Can enteric methane emissions from ruminants be lowered without lowering their production?”, Animal Feed Science and Technology, Vol. 166-167, pp.308-320.

Graus, W. et al. (2004), Marginal GHG-abatement Curves for Agriculture. Ecofys, Utrecht, Pays-Bas. 
Hardtlein, M. et L. Eltrop (2013), "Schwachstellen-Identifikation und Optimierungspotenziale von Biogasanlagen in Baden-Württemberg - Eine technisch-ökonomische Analyse auf der Basis einer Befragung von Biogasanlagenbetreibern”, Universität Stuttgart, Institut für Energiewirtschaft und Rationelle Energieanwendung, Allemagne.

Hasegawa, T. et Y. Matsuoka (2010), "Global methane and nitrous oxide emissions and reduction potentials in agriculture", Journal of Integrative Environmental Sciences, Vol.7 (sup1), pp. 245-256.

Hediger, W. (2006)," Modeling GHG emissions and carbon sequestration in Swiss agriculture: an integrated economic approach Greenhouse Gases and Animal Agriculture: An Update”, Proceedings of the 2nd International Conference on Greenhouse Gases and Animal Agriculture (Zurich, Suisse), International Congress Series, Vol. 1293, pp. 86-95.

Hartmann, M. et al. (2007), "Reducing nitrogen losses from agricultural systems - an integrated economic assessment. $47^{\mathrm{e}}$ conférence annuelle de la GEWISOLA (association allemande des économistes de l'agriculture).

Henderson, B. et al. (à paraître), Marginal costs of abating greenhouse gases in the global ruminant livestock sector. Mitigation and Adaptation Strategies for Global Change.

HGCA (2009), Be PRECISE case studies. http://www. hgca. com/publications/2009/june/03/be-precisecase-studies. aspx, dernière consultation le 1/10/2014.

Hoglund-Isaksson, L. et al. (2010), "Potentials and costs for mitigation of non- $\mathrm{CO}_{2}$ greenhouse gas emissions in the European Union until 2030. Results ", RAPPORT externe à la DG Action pour le climat, International Institute for Applied Systems Analysis, Laxenbourg, Autriche. http://ec.europa.eu/clima/policies/package/docs/non_co2emissions_may2010_en.pdf

Hristov, A. et al. (2013), "Mitigation of greenhouse gas emissions in livestock production - A review of technical options for non- $\mathrm{CO}_{2}$ emissions", Étude FAO : production et santé animales, 177, Rome.

IBERS (The Institute of Biological, Environmental and Rural Sciences) (2010), "Ruminant Nutrition Regimes to Reduce Methane \& Nitrogen Emissions", Rapport no AC0209 du ministère de l'Environnement, de l'alimentation et des affaires rurales, Royaume-Uni.

ICF (2008), "Analysis of the Potential and Costs for Greenhouse Gas Emission Reductions within the New Zealand Agricultural Sector", Rapport international de l'ICF au ministère de l'Agriculture et de la sylviculture, Wellington, Nouvelle-Zélande.

ICF International (2013), “Greenhouse Gas Mitigation Options and Costs for Agricultural Land and Animal Production within the United States", préparé pour le ministère de l'Agriculture des États-Unis, bureau du programme relatif au changement climatique, ICF International, Washington.

IGER (2001), "Cost Curve Assessment of Mitigation Options in Greenhouse Gas Emissions from Agriculture", Rapport n ${ }^{\circ} \mathrm{CC} 0229$ du ministère de l'Environnement, de l'alimentation et des affaires rurales, Royaume-Uni.

Iho, A. (2005), "Does scale matter? Cost-effectiveness of agricultural nutrient abatement when target level varies", Agricultural and Food Science, Vol. 14 (3), pp. 277-295.

Jacobsen, P. (2010), "Natural Breeding versus Artificial Insemination: A Cost Comparison Analysis", University of Wyoming, États-Unis. www.uwyo.edu/agecon/what $\% 20 \mathrm{we} \% 20 \mathrm{do} /$ senior\%20theses/2010_03_29 seniorthesis_pjacobsen.pdf

Jensen, E.S. et al. (2010), "Faba bean in cropping systems”, Field Crops Research, Vol. 115, pp. 203-216.

Jones, C.A., et al. (2013), "New Uses of Old Tools? Greenhouse Gas Mitigation with Agriculture Sector Policies", Applied Economic Perspectives and Policy, Vol. 35 ( 3), pp. 398-434.

Kalamaras, S.D. and T.A. Kotsopoulos (2014), "Anaerobic co-digestion of cattle manure and alternative crops for the substitution of maize in South Europe”, Bioresource Technology, Vol. 172, pp.68-75. 
Keller, M.S. et al. (2006), "Cost-effectiveness of greenhouse gases emission reductions in various sectors", INFRAS, Berne, Suisse.

Kesicki, F. et N. Strachan (2011), "Marginal abatement cost (MAC) curves: confronting theory and practice", Environmental Science \& Policy, Vol. 14, pp. 1195-1204.

Kesicki, F. et P. Ekins (2012), "Marginal abatement cost curves: a call for caution”, Climate Policy, Vol. 12, pp. 219-236.

Key, Nigel, et S. Sneeringer (2011), "Carbon Prices and the Adoption of Methane Digesters on Dairy and Hog Farms", EB-1, ministère de l'Agriculture des États-Unis, Economic Research Service, février 2011.

Kirk, G. et al. (2012), "Quantification of the potential changes in soil carbon in England from soil protection measures within the Soil Protection Review 2010", rapport final du sous-projet A du projet SP1106 du ministère de l'Environnement, de l'alimentation et des affaires rurales : Soil carbon studies to explore greenhouse gas emissions and mitigation, Royaume-Uni.

Konidari, P. et D. Mavrakis (2007), “A multi-criteria evaluation method for climate change mitigation policy instruments”, Energy Policy, Vol. 35, pp. 6235-6257.

Lazarus, W.F. et M. Rudstrom (2007), “The Economics of Anaerobic Digester Operation on a Minnesota Dairy Farm”, Applied Economic Perspectives and Policy, Vol. 29, pp.349-364.

Leip, A. et al. (2010), "Evaluation of the livestock sector's contribution to the EU greenhouse gas emissions (GGELS)", Rapport final. Commission européenne, Centre commun de recherche.

Lengers, B et al. (2014), "What drives marginal abatement costs of greenhouse gases on dairy farms? A meta-modelling approach", Journal of Agricultural Economics, Vol. 65 (3), pp. 579-599.

Lengers, B. et al.(2013), "Comparison of GHG-Emission Indicators for Dairy Farms with Respect to Induced Abatement Costs, Accuracy, and Feasibility", Applied Economic Perspectives and Policy, Vol. 35 (3), pp. 451-475".

Lesschen, J.P. et al. (2011), "Greenhouse gas emission profiles of European livestock sectors", Animal Feed Science and Technology, Vol. 166-167, pp.16-28.

Lewandrowski, J et al. (2004), "Economics of Sequestering Carbon in the U.S. Agricultural Sector", Technical Bulletin No. (TB1909). Washington, DC: U.S. Department of Agriculture, Economic Research Service.

Linkov, I. et al. (2006), "From comparative risk assessment to multi-criteria decision analysis and adaptive management: Recent developments and applications", Environment International, Vol. 32, pp.10721093.

Lu, Y.C. et al. (2000), "Cover crops in sustainable food production", Food Reviews International, Vol. 16, pp.121-157.

Lucas, P. L. et al. (2007), "Long-term reduction potential of non- $\mathrm{CO}_{2}$ greenhouse gases", Environmental Science \& Policy, Vol. 10 (2), pp. 85-103.

MacLeod, M. et al. (2009), Understanding the costs of environmental regulation in Europe , Edward Elgar, Cheltenham, Royaume-Uni.

MacLeod et al. (2010a), "Developing greenhouse gas marginal abatement cost curves for agricultural emissions from crops and soils in the UK", Agricultural Systems, Vol. 103, pp. 198-209.

MacLeod, M. et al. (2010b), "Review and update of UK marginal abatement cost curves for agriculture", Report to Committee on Climate Change, Committee on Climate Change, Londres.

MacLeod, M. et al. (2010c), "Roadmaps Integrating RTD in Developing Realistic GHG Mitigation Options from Agriculture up to 2030", Project Code: AC0214 Final report 28/5/2010, Londres : ministère de l'Environnement, de l'alimentation et des affaires rurales, Royaume-Uni. 
MacLeod, M. et al. (2013), "Greenhouse gas emissions from pig and chicken supply chains - A global life cycle assessment”, FAO, Rome.

MacLeod, M. et al. (2014), "Measuring Progress Towards a Low Carbon Rural Economy", In Agriculture and the Environment X, Delivering Multiple Benefits from our Land: Sustainable Development in Practice, pp. 215-19.

Marette, S. et G. Millet (2014), "Economic benefits from promoting linseed in the diet of dairy cows for reducing methane emissions and improving milk quality", Food Policy, Vol.46, pp.140-149.

Martin, C. et al. (2010), "Methane mitigation in ruminants: from microbe to the farm scale", Animal, Vol. 4, pp. 351-365.

McCarl, B.A. et U.A. Schneider (2001), "Greenhouse gas mitigation in U.S. agriculture and forestry", Science, Vol. 294, pp.2481-82.

McCarl, B.A. et R.D. Sands (2007), "Competitiveness of terrestrial greenhouse gas offsets: are they a bridge to the future?”, Climatic Change, Vol. 80, pp. 109-126.

McKinsey \& Co Climate Desk (2009), "Pathways to a low-carbon economy", Version 2 of the Global Greenhouse Gas Abatement Cost Curve, McKinsey, Chicago.

Merino, P. et al. (2002), "Mitigation of $\mathrm{N}_{2} \mathrm{O}$ emissions from grassland by nitrification inhibitor and Actilith F2 applied with fertilizer and cattle slurry", Soil Use and Management, Vol. 18, pp.135-141.

Miglior, F. et al. (2005), "Selection Indices in Holstein Cattle of Various Countries", Journal of Dairy Science, Vol. 88, pp.1255-1263.

Moate, P.J. et al. (2011), "Influence of cold-pressed canola, brewers grains and hominy meal as dietary supplements suitable for reducing enteric methane emissions from lactating dairy cows", Animal Feed Science and Technology, Vol. 166-167. pp254-264.

Monteny, G. et al. (2006), "Greenhouse gas abatement strategies for animal husbandry", Agriculture, Ecosystems \& Environment, Vol. 112 (2-3),pp.163-170.

Moorby, J., et al. (2007), "A review of research to identify best practice for reducing greenhouse gases from agriculture and land management", IGER-ADAS, rapport $\mathrm{n}^{\circ} \mathrm{AC0206}$ du ministère de l'Environnement, de l'alimentation et des affaires rurales, Royaume-Uni.

Moran, D. et al. (2011), "Developing carbon budgets for UK agriculture, land-use, land-use change and forestry out to 2022”, Climatic Change, Vol. 105, pp.529-553.

Moran, D. et al. (2011), "Marginal Abatement Cost Curves for UK Agricultural Greenhouse Gas Emissions", Journal of Agricultural Economics, Vol. 62, pp.93-118.

Moran, D. et al. (2008), "UK marginal abatement cost curves for the agriculture and land use, land-use change and forestry sectors out to 2022, with qualitative analysis of options to 2050", Rapport final. Comité britannique sur le changement climatique, Londres. http://core.ac.uk/download/pdf/6557621.pdf

Moran, D. et al. (2012), "Wider implications of greenhouse gas mitigation measures in English agriculture", Rapport n ${ }^{\circ}$ AC0226 du ministère de l'Environnement, de l'alimentation et des affaires rurales, Royaume-Uni.

Moran, D. et al. (2013), "Mitigation win-win", Nature Climate Change, Vol. 3., pp. 611-13.

Myhre, G.D. et al. (2013), "Anthropogenic and Natural Radiative Forcing”, In : Climate Change 2013 : The Physical Science Basis. Contribution of Working Group I to the Fifth Assessment Report of the Intergovernmental Panel on Climate Change [Stocker, T.F. et al. (éds.)], Cambridge University Press, Cambridge, Royaume-Uni et New York, États-Unis.

Naucler, T. et P.A. Enkvist (2009) "Pathways to Low Carbon Economy: Version 2 of the Global Greenhouse Gas Abatement Cost Curve", McKinsey \& Company. 
Nemecek, T et al. (2008), "Environmental impacts of introducing grain legumes into European crop rotations", European Journal of Agronomy, Vol. 28, pp. 380-393.

Nemet, G. et al. (2010), "Implications of incorporating air-quality co-benefits into climate change policymaking”, Environmental Research Letters, Vol.5, p.9.

Neufeldt, H. et M. Schafer (2008), "Mitigation strategies for greenhouse gas emissions from agriculture using a regional economic-ecosystem model”, Agriculture Ecosystems \& Environment, Vol. 123 (4), pp.305-316.

Nordhaus, W. et J. Boyer (1999), Warming the World - Economic Models of Global Warming, Internet Edition, MIT Press, États-Unis.

OCDE (2008), Handbook on Constructing Composite Indicators, OCDE, Paris.

OCDE (2014), Compendium des indicateurs agro-environnementaux de l'OCDE, Éditions OCDE, Paris. dx.doi.org/10.1787/9789264181243-fr .

OCDE (2015), Indicateurs de croissance verte pour l'agriculture : Évaluation préliminaire, Études de l'OCDE sur la croissance verte, Éditions OCDE, Paris. dx.doi.org/10.1787/9789264226111-fr

O’Hara, P., J. Freney et M. Ulyatt (2003), « Abatement of Agricultural Non-Carbon Dioxide Greenhouse Gas Emissions », ministère de l'Agriculture et de la sylviculture, Wellington, Nouvelle-Zélande.

Opio, C. et al. (2013), "Greenhouse gas emissions from ruminant supply chains - A global life cycle assessment”, Food and Agriculture Organization of the United Nations (FAO), Rome.

Pannell, D. (2008), "Public benefits, private benefits, and policy mechanism choice for land use change for environmental benefits", Land Economics, Vol. 84(2), pp. 225-240.

Patra, A. (2012), "Enteric methane mitigation technologies for ruminant livestock: a synthesis of current research and future directions", Environ Monitoring Assessment, Vol. 184, pp.1929-1952.

Pearce, D.W. et R.K. Turner (1990), Economics of natural resources and the environment, Harvester Wheatsheaf, Londres, Royaume-Uni.

Pellerin S. et al. (2013), «Quelle contribution de l'agriculture française à la réduction des émissions de gaz à effet de serre ? Coût et potentiel de réduction de la pollution de dix mesures techniques : le cas de la France », Synthèse du rapport d'étude, INRA, France.

Perez, I. (2004), “Greenhouse gases: Inventories, abatement costs and markets for emission permits in European agriculture - A modelling approach”, PhD Dissertation, Rheinischen Friedrich-WilhelmsUniversität zu Bonn, Bonn, Allemagne.

Pike, T. (2008), "Understanding behaviours in a farming context: bringing theoretical and applied evidence together from across Defra and highlighting policy relevance and implications for future research", novembre 2008, document de travail de l'Observatoire de l'environnement et du changement agricole du ministère de l'Environnement, de l'alimentation et des affaires rurales, Londres : ministère de l'Environnement, de l'alimentation et des affaires rurales, Royaume-Uni.

Pillars, R. (2014), "Farm based anaerobic digesters", Manure Technologies Fact Sheet Series, 1-2. Michigan State University Extension, États-Unis.

Pollok C. (2008), “Options for Greenhouse Gas Mitigation in the UK April 2008 Market”, Harborough : Nuffield Farming Scholarships Trust, Royaume-Uni.

Posthumus, H et al. (2013), "Costs and benefits of erosion control measures in the UK", Soil Use and Management, doi: 10.1111/sum.12057

Povellato, A. et al. (2007), "Cost-effectiveness of greenhouse gases mitigation measures in the European agro-forestry sector: a literature survey", Environmental Science \& Policy, Vol. 10(5), pp. 474-490.

Pretty, J.N. et al. (2000), "An assessment of the total external costs of UK agriculture", Agricultural Systems, Vol. 65, pp. 113-136. 
Radov, D. et al. (2007), "Market mechanisms for reducing GHG emissions from agriculture, forestry and land management", NERA Economic Consulting, Londres: ministère de l'Environnement, de l'alimentation et des affaires rurales, Royaume-Uni.

Rains, G.C. et D.L. Thomas (2009), "Precision Farming - An Introduction". Bulletin 1186, The Cooperative Extension Service, University of Georgia, Etats-Unis.

Rees, R.M. et al. (2014). "Legume Futures Final Report. 2014”. SRUC, Edinburgh. www.legumefutures.de

Regina, K. et al. (2013), "Emissions of nitrous oxide from boreal agricultural mineral soils-Statistical models based on measurements", Agriculture, Ecosystems and Environment, Vol. 164, pp. 131-136.

Reichardt, M. et C. Jurgens (2009), “Adoption and future perspective of precision farming in Germany: results of several surveys among different agricultural target groups", Precision Agriculture, Vol. 10, pp.73-94.

Reichardt, M.C. et al. (2009), "Dissemination of precision farming in Germany: acceptance, adoption, obstacles, knowledge transfer and training activities", Precision Agriculture, Vol. 10, pp.525-545.

Reisinger, A. et S. Ledgard (2013), "Impact of greenhouse gas metrics on the quantification of agricultural emissions and farm-scale mitigation strategies: A New Zealand case study", Environmental Research Letters, Vol. 8.

Schils, R.L.M. et al. (2007), "DairyWise, A Whole-Farm Dairy Model”, Journal of Dairy Science, Vol. 90 (11), pp. 5334-5346.

Schneider, U.A. et B.A. McCarl (2006), “Appraising agricultural greenhouse gas mitigation potentials: Effects of alternative assumptions”, Agricultural Economics, Vol. 35(3), pp.277-287.

Schneider, U.A et al. (2007), "Agricultural sector analysis on greenhouse gas mitigation in US agriculture and forestry”, Agricultural Systems, Vol. 94, pp. 128-140.

Schneider, U.A. (2000), “Agricultural sector analysis on greenhouse gas mitigation in US agriculture and forestry”, Dissertation, Texas A\&M University, Etats-Unis.

Schulte, R.P. et al. (2012), “A Marginal Abatement Cost Curve for Irish Agriculture”, Teagasc, Dublin, Irlande.

Sikirica, N. et al. (2014), "Overview of mitigation measures in agriculture for AGLINK-COSIMO", Wageningen : Alterra Wageningen UR, Pays-Bas.

Smith, K.A. et al. (1997), "Emissions of $\mathrm{N}_{2} \mathrm{O}$ and $\mathrm{NO}$ associated with nitrogen fertilization in intensive agriculture, and the potential for mitigation”. Soil Use and Management, Vol.13, pp. 296-304.

Smith, P.D. et al. (2008), "Greenhouse gas mitigation in agriculture", Philosophical Transactions of the Royal Society B-Biological Sciences, Vol. 363, pp.789-813.

Smith P. et al. (2014), «Agriculture, Forestry and Other Land Use (AFOLU) », in Edenhofer, O. et al. (éds.), Climate Change 2014: Mitigation of Climate Change. Contribution of Working Group III to the Fifth Assessment Report of the Intergovernmental Panel on Climate Change, Cambridge University Press, Cambridge, Royaume-Uni et New York, États-Unis.

Stavins, R.N. et K.R. Richards (2005), “The cost of U.S. forest-based carbon sequestration”, Pew Center on Global Climate Change, Washington DC.

Stern, D. I. et al. (2012), "Where in the world is it cheapest to cut carbon emissions?", Australian Journal of Agricultural and Resource Economics, Vol. 56 (3), pp. 315-331.

Steve Sorrell (2007), «The Rebound Effect: an assessment of the evidence for economy-wide energy savings from improved energy efficiency », rapport produit par le Sussex Energy Group pour la fonction «Technologie et évaluation stratégique» du Centre britannique de recherche sur l'énergie, octobre 2007, Londres : UKERC, Royaume-Uni. 
Sustainable Energy Ireland (2009), "Ireland's Low-Carbon Opportunity: An analysis of the costs and benefits of reducing greenhouse gas emissions: Technical Appendix”, Dublin: Sustainable Energy, Irlande.

The Andersons Centre (2010), “A detailed economic assessment of anaerobic digestion technology and its suitability to UK farming and waste systems. $2^{\mathrm{e}}$ edition”, The Andersons Centre, Royaume-Uni.

USEPA (2013), “Global Mitigation of Non-CO2 Greenhouse Gases: 2010-2030.”, EPA-430-R-13-011, US EPA, Washington DC. www.epa.gov/climatechange/EPAactivities/economics/nonco2mitigation.html.

Valantin, G. (2012), "Marginal abatement cost curves for UK forestry", Forestry Commission Research Report. Édimbourg : Commission Foresterie, Royaume-Uni.

van Amstel, A.R. (2012), "Methane: its role in climate change and options for control", Dissertation. Université de Wageningue, Pays-Bas.

van den Pol-Dasselaar, A. et al. (2013), "Kosteneffectiviteit reductiemaatregelen emissie broeikasgassen zuivel”, Wageningen UR Livestock Research, Lelystad, Pays-Bas.

van Groenigen, J.W. et al. (2008), "Mitigation strategies for greenhouse gas emissions from animal production systems: synergy between measuring and modelling at different scales", Australian Journal of Experimental Agriculture, Vol. 48 (2), pp. 46-53.

Vellinga, T.V. et al. (2011), "Implementation of GHG mitigation on intensive dairy farms: Farmers' preferences and variation in cost effectiveness", Livestock Science, Vol. 137 (1-3), pp. 185-195.

Vellinga, T.V. et al. (2013), "Methodology used in FeedPrint: a tool quantifying greenhouse gas emissions of feed production and utilization", rapport 674, Lelystad : WUR, Pays-Bas.

Vermont, B. et S. De Cara (2010), "How costly is mitigation of non- $\mathrm{CO}_{2}$ greenhouse gas emissions from agriculture?: A meta-analysis “, Ecological Economics 69 : pp. 1373-1386.

Wagner, F. et al. (2012), "Sectoral marginal abatement cost curves: implications for mitigation pledges and air pollution co-benefits for Annex I countries”, Sustainable Science, Vol. 7 (2), pp. 169-184.

Wall, E., et al. (2010), "Developing breeding schemes to assist mitigation of greenhouse gas emissions", Animal, Vol. 4, pp.366-376.

Walla, C. et W. Schneeberger (2008), “The optimal size for biogas plants", Biomass and Bioenergy, Vol. 32, pp.551-557.

Wallander, S. (2013) "While Crop Rotations Are Common, Cover Crops Remain Rare" Amber Waves, USDA Economic Research Services, Washington. www.ers.usda.gov/amber-waves/2013-march/whilecrop-rotations-are-common,-cover-crops-remain-rare.aspx, dernière consultation le 13/1/15

Wassmann, R. et H. Pathak (2007), "Introducing greenhouse gas mitigation as a development objective in rice-based agriculture: II. Cost-benefit assessment for different technologies, regions and scales", Agricultural Systems, Vol. 94 (3) pp. 826-840.

Weiske, A. et J. Michel (2007), "Greenhouse gas emissions and mitigation costs of selected mitigation measures in agricultural production”, MEACAP WP3 D15a, Institute for Energy and Environment (IE), Leipzig.

Weyant, J.P. et al. (2006), “Overview of EMF-21: Multigas Mitigation and Climate Policy”, The Energy Journal, Vol 27, pp. 1-32.

Whittle, L. et al. (2013), "Costs and potential of agricultural emissions abatement in Australia: A quantitative assessment of livestock abatement under the CFI", ABARES technical report 13.2, ABARes, Canberra.

Wilkinson, K.G. (2011), “A comparison of the drivers influencing adoption of on-farm anaerobic digestion in Germany and Australia”, Biomass and Bioenergy, Vol. 35, pp.1613-1622. 
Wiltshire. J. et al. (2014), “Assessment of the Effectiveness, Impact and Cost of Measures to Protect Soils: Report for Defra”.. Didcot : Ricardo-AEA Ltd, Royaume-Uni.

Winiwarter, W. et Z. Klimont (2011), "The role of N-gases (N2O, NOx, NH3) in cost-effective strategies to reduce greenhouse gas emissions and air pollution in Europe", Current Opinion in Environmental Sustainability, Vol. 3, 438-445.

Wreford, A. et al. (2010), Climate Change and Agriculture: Impacts, adaptation and mitigation. OCDE, Paris. dx.doi.org/10.1787/9789264086876-en

Yiridoe, E.K. et al. (2009), "Nonmarket co-benefits and economic feasibility of on-farm biogas energy production”, Energy Policy, Vol. 37, pp.1170-1179. 


\section{Annexe A.}

\section{Évolution des émissions de GES dans les pays de l'OCDE}

Graphique 8. Émissions de gaz à effet de serre $\left(\mathrm{CH}_{4}\right.$ et $\left.\mathrm{N}_{2} \mathrm{O}\right)$ d'origine agricole, pays de l'OCDE, 1990-2010

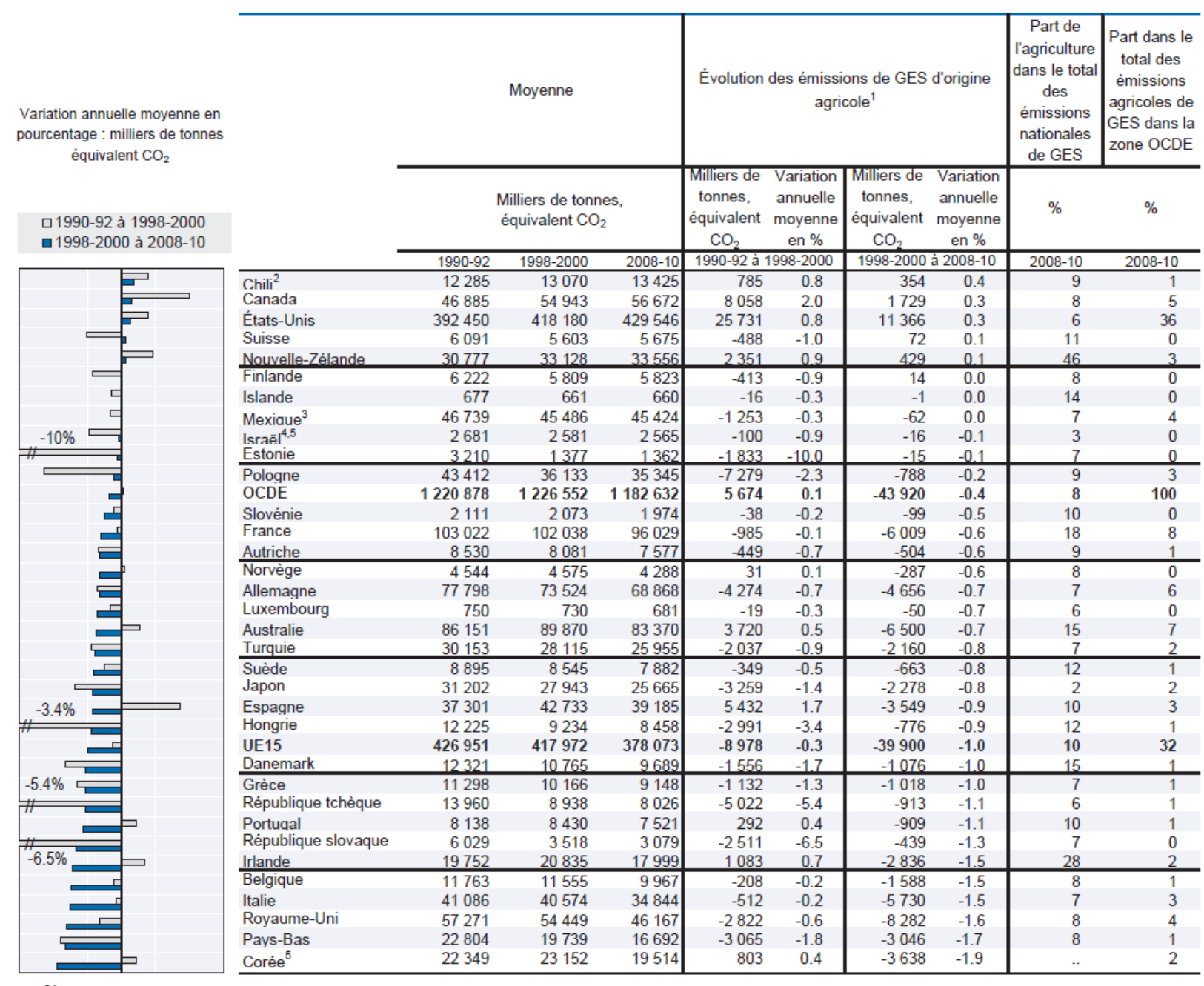

Source : OCDE (2013). 
Annexe B.

\section{Complément d'information}

Tableau 26. Exemples de coûts (-) et avantages (+) secondaires de certaines mesures courantes d'atténuation des GES

\begin{tabular}{|c|c|c|c|c|c|c|c|c|c|c|c|c|}
\hline Effet global & + & + & + & - & - & - & - & - & - & - & - & \\
\hline Mesure & 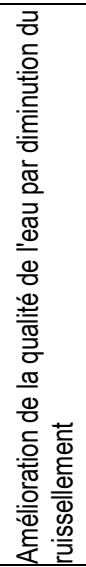 & 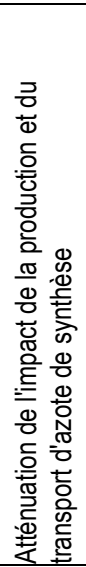 & 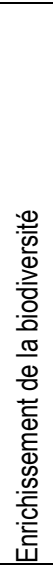 & 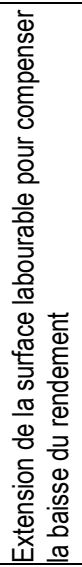 & 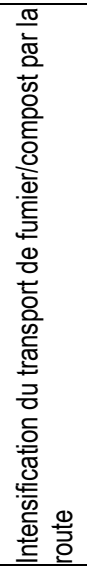 & 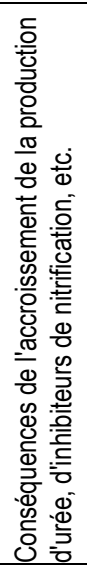 & 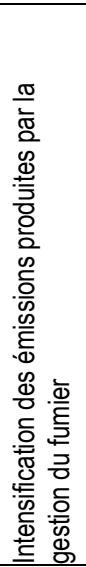 & 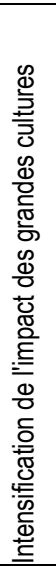 & 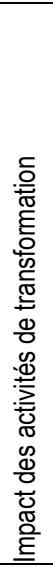 & 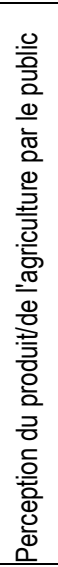 & 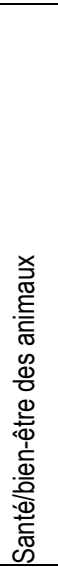 & Autres effets \\
\hline $\begin{array}{l}\text { Fixation biologique utilisée comme source } \\
\text { d'azote }\end{array}$ & $\mathrm{x}$ & $\mathrm{x}$ & $\mathrm{x}$ & $\mathrm{x}$ & & & & & & & & \\
\hline Baisse de l'utilisation d'engrais azotés & $x$ & $\mathrm{x}$ & & $\mathrm{x}$ & & & & & & & & \\
\hline Amélioration du drainage des sols & $\mathrm{x}$ & & & & & & & & & & & \\
\hline Éviter l'excès d'azote & $x$ & $\mathrm{x}$ & & & & & & & & & & \\
\hline $\begin{array}{l}\text { Prise en compte de l'apport total en azote } \\
\text { du fumier }\end{array}$ & $x$ & $x$ & & $\mathrm{x}$ & $\mathrm{x}$ & & & & & & & \\
\hline $\begin{array}{l}\text { Amélioration du calendrier de l'épandage } \\
\text { d'engrais azotés de synthèse }\end{array}$ & $\mathrm{x}$ & $\mathrm{x}$ & & & & & & & & & & \\
\hline Engrais à libération contrôlée & & & & & & $\mathrm{x}$ & & & & & & $\begin{array}{l}\text { Risque accru de } \\
\text { lessivage de } \\
\text { l'azote }\end{array}$ \\
\hline Inhibiteurs de nitrification & $\mathrm{x}$ & $\mathrm{x}$ & & & & $\mathrm{x}$ & & & & & & \\
\hline $\begin{array}{l}\text { Amélioration du calendrier de l'épandage } \\
\text { de lisier et de fientes de volaille }\end{array}$ & $\mathrm{x}$ & $\mathrm{x}$ & & & & & $\mathrm{x}$ & & & & & \\
\hline $\begin{array}{l}\text { Adoption de systèmes moins dépendants } \\
\text { des intrants }\end{array}$ & $\mathrm{x}$ & $\mathrm{x}$ & $\mathrm{x}$ & $\mathrm{x}$ & & & & & & & & \\
\hline $\begin{array}{l}\text { Obtentions végétales à assimilation de } \\
\text { l'azote renforcée }\end{array}$ & $\mathrm{x}$ & $\mathrm{x}$ & & & & & & & & & & \\
\hline $\begin{array}{l}\text { Séparation des épandages de lisier et } \\
\text { d'engrais }\end{array}$ & $\mathrm{x}$ & & & & & & & & & & & \\
\hline $\begin{array}{l}\text { Allègement ou suppression du travail du } \\
\text { sol }\end{array}$ & $\mathrm{x}$ & & & & & & & & & & & $\begin{array}{l}\text { Atténuation de } \\
\text { l'érosion du sol }\end{array}$ \\
\hline $\begin{array}{l}\text { Utilisation de compost et de fumier } \\
\text { pailleux de préférence au lisier }\end{array}$ & & $\mathrm{x}$ & & & $x$ & & $x$ & & & & & \\
\hline $\begin{array}{l}\text { Accroissement de la teneur en amidon de } \\
\text { l'alimentation }\end{array}$ & & & & & & & & $x$ & & & $\mathrm{x}$ & \\
\hline $\begin{array}{l}\text { Hausse de la part de maïs-ensilage dans } \\
\text { l'alimentation }\end{array}$ & & & & & & & & $\mathrm{x}$ & & & & \\
\hline Précurseurs d'acide propionique & & & & & & & & & $\mathrm{x}$ & $x$ & & \\
\hline Probiotiques & & & & & & & & & $\mathrm{x}$ & $\mathrm{x}$ & & \\
\hline lonophores & & & & & & & & & $\mathrm{x}$ & $x$ & & \\
\hline
\end{tabular}


Tableau 26. Exemples de coûts (-) et avantages (+) secondaires de certaines mesures courantes d'atténuation des GE (suite)

\begin{tabular}{lcc}
\hline Somatotropine bovine & $\mathrm{x}$ \\
$\begin{array}{l}\text { Potentiel génétique amélioré des vaches } \\
\text { laitières - productivité }\end{array}$ & $\mathrm{x}$ \\
$\begin{array}{l}\text { Potentiel génétique amélioré des vaches } \\
\text { laitières - fertilité }\end{array}$ & $\mathrm{x}$ \\
$\begin{array}{l}\text { Potentiel génétique amélioré des bovins à } \\
\text { viande }\end{array}$ & $\mathrm{x}$ \\
Manipulation transgénique des ruminants & $\mathrm{x}$ \\
\hline
\end{tabular}

Source : d'après Moran et al., 2008, Annexe B.

Tableau 27. Études récentes du rapport coût-efficacité de mesures multiples d'atténuation des émissions de GES

Méthode de calcul du rapport coût-efficacité : 1. études d'estimation ascendante des coûts ; 2. modélisation microéconomique ; 3. modèles d'équilibre axés sur l'offre, par région ou par secteur.

\begin{tabular}{|c|c|c|c|c|c|c|c|}
\hline $\mathrm{N}^{\circ}$ & Étude & $\begin{array}{c}\text { Champ } \\
\text { géographique } \\
\text { couvert }\end{array}$ & Résolution & $\begin{array}{c}\text { Au niveau de } \\
\text { l'exploitation } \\
\text { ou du cycle } \\
\text { de vie } \\
\end{array}$ & $\begin{array}{c}\text { Méthode } \\
\text { d'obtention } \\
\text { de la MACC }\end{array}$ & $\begin{array}{l}\text { Interac- } \\
\text { tions }\end{array}$ & $\begin{array}{c}\text { Rend compte du } \\
\text { rapport CE des } \\
\text { mesures prises } \\
\text { séparément? }\end{array}$ \\
\hline 1 & Schneider 2000 & États-Unis & Régions infranationales & exploitation & 3 & $\begin{array}{l}\text { Non } \\
\text { précisé }\end{array}$ & Non \\
\hline 4 & IGER 2001 & Royaume-Uni & $\begin{array}{l}\text { De l'échelle d'une } \\
\text { exploitation à l'échelle } \\
\text { nationale }\end{array}$ & exploitation & 1 & $\begin{array}{l}\text { Non } \\
\text { précisé }\end{array}$ & Oui \\
\hline 6 & Graus et al. 2004 & Monde & Régions mondiales & exploitation & 1 & Oui & Oui \\
\hline 7 & Perez 2004 & UE des 15 & NUTS 2 & exploitation & 3 & Non & Non \\
\hline 9 & De Cara et al. 2005 & UE des 15 & Régionale (NUTS 2) & exploitation & 2 & Non & Non \\
\hline 11 & $\begin{array}{l}\text { DeAngelo et al., } \\
2006\end{array}$ & Monde & Régions mondiales & exploitation & 1 & $\begin{array}{l}\text { Évite le } \\
\text { double } \\
\text { comptage }\end{array}$ & Non \\
\hline 12 & $\begin{array}{l}\text { Hediger, } 2006 \text { et } \\
\text { Hartmann, } 2007\end{array}$ & Suisse & Régions infranationales & exploitation & 2 & $\begin{array}{l}\text { Non } \\
\text { précisé }\end{array}$ & Non \\
\hline 15 & Lucas et al., 2007 & Monde & Non précisé & $\begin{array}{l}\text { exploitation + } \\
\text { autres } \\
\text { secteurs }\end{array}$ & 1 & Non & Non \\
\hline 18 & Schils et al., 2007 & Pays-Bas & Exploitation & exploitation & 2 & Non & Non \\
\hline 19 & $\begin{array}{l}\text { Schneider et al., } \\
2007\end{array}$ & États-Unis & Régions infranationales & exploitation & 3 & Non & Non \\
\hline 20 & $\begin{array}{l}\text { Wassman et } \\
\text { Pathak, } 2007\end{array}$ & $\begin{array}{l}\text { Philippines, Chine, } \\
\text { Inde }\end{array}$ & Régions infranationales & exploitation & 1 & $\begin{array}{l}\text { Non } \\
\text { précisé }\end{array}$ & Non \\
\hline 21 & Weiske et al., 2007 & $\begin{array}{l}\text { Nord-Ouest de } \\
\text { l'Allemagne }\end{array}$ & $\begin{array}{l}\text { Au niveau de } \\
\text { l'exploitation }\end{array}$ & exploitation & 3 & $\begin{array}{l}\text { Non } \\
\text { précisé }\end{array}$ & Oui \\
\hline 22 & Amann et al., 2008 & UE des 27 & Nationale & exploitation & 3 & $\begin{array}{l}\text { Évite le } \\
\text { double } \\
\text { comptage }\end{array}$ & Oui \\
\hline 23 & Beach et al., 2008 & Monde & Monde & exploitation & 1 & $\begin{array}{l}\text { Évite le } \\
\text { double } \\
\text { comptage }\end{array}$ & Non \\
\hline 24 & EPA, 2008 & Queensland & Queensland & exploitation & 3 & Oui & Non \\
\hline 25 & ICF ; 2008 & Nouvelle-Zélande & Nationale & exploitation & 1 & $\begin{array}{l}\text { Non } \\
\text { précisé }\end{array}$ & Oui \\
\hline 26 & Moran et al., 2008 & Royaume-Uni & Nationale & exploitation & 1 et 2 & Oui & Oui \\
\hline 27 & $\begin{array}{l}\text { Neufeldt et Shcafer, } \\
2008\end{array}$ & Allemagne & Régionale & exploitation & 2 & Oui & Non \\
\hline 28 & Smith et al., 2008 & Monde & Régions mondiales & exploitation & 1 & $\begin{array}{l}\text { Non } \\
\text { précisé }\end{array}$ & Non \\
\hline 30 & Bates et al., 2009 & UE des 27 & UE des 27 & exploitation? & 1 & $\begin{array}{l}\text { Non } \\
\text { précisé }\end{array}$ & Oui \\
\hline
\end{tabular}




\begin{tabular}{|c|c|c|c|c|c|c|c|}
\hline $\mathrm{N}^{0}$ & Étude & $\begin{array}{c}\text { Champ } \\
\text { géographique } \\
\text { couvert }\end{array}$ & Résolution & $\begin{array}{c}\text { Au niveau de } \\
\text { l'exploitation } \\
\text { ou du cycle } \\
\text { de vie }\end{array}$ & $\begin{array}{l}\text { Méthode } \\
\text { d'obtention } \\
\text { de la MACC }\end{array}$ & $\begin{array}{l}\text { Interac- } \\
\text { tions }\end{array}$ & $\begin{array}{l}\text { Rend compte du } \\
\text { rapport CE des } \\
\text { mesures prises } \\
\text { séparément? }\end{array}$ \\
\hline 31 & Golub et al., 2009 & Monde & Régions mondiales & exploitation & 3 & Non & Non \\
\hline 32 & McKinsey, 2009 & Monde & Monde & exploitation & 1 & $\begin{array}{l}\text { Non } \\
\text { précisé }\end{array}$ & Non \\
\hline 33 & $\begin{array}{l}\text { AEA Technologies, } \\
2010\end{array}$ & Angleterre & Nationale & exploitation & 1 & Oui & Oui \\
\hline 34 & $\begin{array}{l}\text { Durandeau et al., } \\
2010\end{array}$ & France & Régions infranationales & exploitation & 2 & Oui & Non \\
\hline 35 & $\begin{array}{l}\text { Hasegawa et al., } \\
2010\end{array}$ & Monde & Régions mondiales & exploitation & 3 & Oui & Oui \\
\hline 36 & $\begin{array}{l}\text { Hoglund-Isaksson } \\
\text { et al., } 2010\end{array}$ & $\begin{array}{l}\text { Pays signataires } \\
\text { de l'annexe I du } \\
\text { protocole de Kyoto }\end{array}$ & Nationale & $\begin{array}{l}\text { exploitation + } \\
\text { autres } \\
\text { secteurs }\end{array}$ & 1 & Oui & Oui \\
\hline 37 & IBERS 2010 & Royaume-Uni & $\begin{array}{l}\text { De l'échelle d'une } \\
\text { exploitation à l'échelle } \\
\text { nationale }\end{array}$ & exploitation & 1 et 2 & Non & Oui \\
\hline 38 & Leip et al., 2010 & UE des 27 & NUTS 2 & ACV & 3 & Oui & Non \\
\hline 41 & $\begin{array}{l}\text { De Cara et Jayet, } \\
2011\end{array}$ & UE des 24 & Régionale (NUTS 2) & exploitation & 2 & Non & Non \\
\hline 45 & Vellinga et al., 2011 & Pays-Bas & $\begin{array}{l}\text { Au niveau de } \\
\text { l'exploitation }\end{array}$ & exploitation & 2 & Non & Oui \\
\hline 46 & $\begin{array}{l}\text { Dominguez et al., } \\
2012\end{array}$ & UE des 27 & NUTS 2 & exploitation & 3 & Oui & Non \\
\hline 47 & Schulte et al., 2012 & Irlande & Nationale & ACV & 1 & Oui & Oui \\
\hline 50 & van Amstel, 2012 & Monde & Monde & exploitation & 3 & Oui & Non \\
\hline 51 & Wagner et al., 2012 & $\begin{array}{l}\text { Pays signataires } \\
\text { de l'annexe I du } \\
\text { protocole de Kyoto }\end{array}$ & Nationale & $\begin{array}{l}\text { exploitation + } \\
\text { autres } \\
\text { secteurs }\end{array}$ & 1 & $\begin{array}{l}\text { Évite le } \\
\text { double } \\
\text { comptage }\end{array}$ & Non \\
\hline 52 & Cornet et al., 2013 & Belgique & Nationale & exploitation & 1 & $\begin{array}{l}\text { Non } \\
\text { précisé }\end{array}$ & Non \\
\hline 54 & ICF, 2013 & États-Unis & $\begin{array}{l}\text { Au niveau de } \\
\text { l'exploitation }\end{array}$ & exploitation & 1 & $\begin{array}{l}\text { Non } \\
\text { précisé }\end{array}$ & Oui \\
\hline 55 & Pellerin et al., 2013 & France & Nationale & $\begin{array}{l}\text { Au niveau de } \\
\text { l'exploitation et } \\
\text { en partie en } \\
\text { amont/aval de } \\
\text { l'exploitation }\end{array}$ & 1 & Oui & Oui \\
\hline 56 & $\begin{array}{l}\text { Reisinger et } \\
\text { Legard, } 2013\end{array}$ & Nouvelle-Zélande & $\begin{array}{l}\text { Au niveau de } \\
\text { l'exploitation }\end{array}$ & $\mathrm{ACV}$ & 1 & $\begin{array}{l}\text { Non } \\
\text { précisé }\end{array}$ & Non \\
\hline 57 & USEPA, 2013 & Monde & $\begin{array}{l}\text { Nationale/supra- } \\
\text { nationale }\end{array}$ & exploitation & 1 & $\begin{array}{l}\text { Évite le } \\
\text { double } \\
\text { comptage }\end{array}$ & Non \\
\hline 59 & Whittle et al., 2013 & Australie & $\begin{array}{l}\text { Au niveau de } \\
\text { l'exploitation }\end{array}$ & exploitation & 1 & $\begin{array}{l}\text { Évite le } \\
\text { double } \\
\text { comp- } \\
\text { tage }\end{array}$ & Non \\
\hline 61 & Doreau et al., 2014 & France & Nationale & exploitation & 1 & Oui & Oui \\
\hline 63 & $\begin{array}{l}\text { Henderson et al., (à } \\
\text { paraître) }\end{array}$ & Monde & $\begin{array}{l}\text { De } 8 \mathrm{~km}^{2} \text { à supra- } \\
\text { nationale }\end{array}$ & ACV & 1 & $\begin{array}{l}\text { Évite le } \\
\text { double } \\
\text { comp- } \\
\text { tage }\end{array}$ & Non \\
\hline 64 & $\begin{array}{l}\text { Lengers et al., } 2013 \\
\text { et Lengers et al., } \\
2014\end{array}$ & Allemagne & $\begin{array}{l}\text { Au niveau de } \\
\text { l'exploitation }\end{array}$ & exploitation & 2 & Oui & Non \\
\hline
\end{tabular}

Source : Références citées. 
66 -RAPPORT COÛT-EFFICACITÉ DES MESURES D’ATTÉNUATION DES ÉMISSIONS DE GAZ À EFFET DE SERRE EN AGRICULTURE

Tableau 28. Synthèse des sous-catégories de mesures d'atténuation incluses dans chaque étude (pour les études comportant un calcul du rapport coût-efficacité) Les nombres indiquent le nombre de mesures d'atténuation dans chaque sous-catégorie mentionnée pour chaque étude

\begin{tabular}{|c|c|c|c|c|c|c|c|c|c|c|c|c|c|c|c|}
\hline Catégorie/No de l'étude & 4 & 6 & 11 & 20 & 21 & 22 & 23 & 24 & 25 & 26 & $\underline{28}$ & 30 & 32 & 33 & 35 \\
\hline Gestion des terres labourables - total & 9 & 9 & 4 & 7 & 4 & 7 & 6 & 2 & 1 & 14 & 8 & 4 & 4 & 2 & 15 \\
\hline Agronomie & 2 & 0 & 0 & 0 & 0 & 1 & 0 & 0 & 0 & 1 & 2 & 0 & 0 & 0 & 0 \\
\hline Gestion des nutriments & 7 & 3 & 2 & 0 & 3 & 4 & 3 & 0 & 1 & 11 & 2 & 4 & 1 & 0 & 6 \\
\hline Modifications des structures et de la gestion & 0 & 0 & 0 & 0 & 1 & 0 & 0 & 0 & 0 & 0 & 0 & 0 & 0 & 0 & 0 \\
\hline Travail du sol et gestion des résidus & 0 & 0 & 0 & 0 & 0 & 1 & 1 & 2 & 0 & 1 & 1 & 0 & 1 & 1 & 1 \\
\hline Gestion de l'eau et des sols & 0 & 0 & 0 & 0 & 0 & 0 & 0 & 0 & 0 & 1 & 2 & 0 & 0 & 1 & 0 \\
\hline Gestion du riz & 0 & 6 & 2 & 7 & 0 & 1 & 2 & 0 & 0 & 0 & 1 & 0 & 2 & 0 & 8 \\
\hline Vergers & 0 & 0 & 0 & 0 & 0 & 0 & 0 & 0 & 0 & 0 & 0 & 0 & 0 & 0 & 0 \\
\hline Gestion des pâturages - total & 0 & 1 & 0 & 0 & 1 & 0 & 1 & 1 & 3 & 1 & 4 & 3 & 0 & 0 & 0 \\
\hline Intensité et calendrier du pâturage & 0 & 1 & 0 & 0 & 1 & 0 & 1 & 0 & 1 & 0 & 1 & 2 & 0 & 0 & 0 \\
\hline Accroissement de la productivité & 0 & 0 & 0 & 0 & 0 & 0 & 0 & 0 & 1 & 1 & 1 & 1 & 0 & 0 & 0 \\
\hline Gestion par le feu & 0 & 0 & 0 & 0 & 0 & 0 & 0 & 1 & 0 & 0 & 2 & 0 & 0 & 0 & 0 \\
\hline Gestion de l'eau et des sols & 0 & 0 & 0 & 0 & 0 & 0 & 0 & 0 & 1 & 0 & 0 & 0 & 0 & 0 & 0 \\
\hline Gestion des sols organiques - total & 0 & 0 & 0 & 0 & 0 & 0 & 0 & 0 & 0 & 0 & 1 & 0 & 1 & 0 & 0 \\
\hline Restauration des terres dégradées - total & 0 & 0 & 0 & 0 & 0 & 0 & 0 & 1 & 0 & 0 & 3 & 0 & 1 & 0 & 0 \\
\hline Gestion du bétail & 10 & 11 & 0 & 0 & 3 & 1 & 6 & 3 & 5 & 11 & 3 & 4 & 2 & 0 & 2 \\
\hline Rendement des nutriments et alimentation & 3 & 6 & 0 & 0 & 3 & 1 & 4 & 1 & 1 & 4 & 1 & 2 & 0 & 0 & 0 \\
\hline Additifs alimentaires et agents spécifiques & 2 & 1 & 0 & 0 & 0 & 0 & 2 & 1 & 2 & 3 & 1 & 0 & 2 & 0 & 2 \\
\hline Santé animale & 0 & 0 & 0 & 0 & 0 & 0 & 0 & 0 & 0 & 0 & 0 & 0 & 0 & 0 & 0 \\
\hline Modifications des structures et de la gestion & 1 & 2 & 0 & 0 & 0 & 0 & 0 & 0 & 1 & 0 & 1 & 1 & 0 & 0 & 0 \\
\hline Structure des troupeaux et génétique animale & 4 & 2 & 0 & 0 & 0 & 0 & 0 & 1 & 1 & 4 & 0 & 1 & 0 & 0 & 0 \\
\hline Stabulation et fumier - total & 5 & 0 & 3 & 0 & 6 & 1 & 1 & 2 & 1 & 3 & 2 & 3 & 0 & 0 & 5 \\
\hline Stabulation - total & 1 & 0 & 0 & 0 & 3 & 0 & 0 & 1 & 0 & 0 & 0 & 0 & 0 & 0 & 0 \\
\hline Amélioration du stockage et de la gestion du fumier & 3 & 0 & 0 & 0 & 2 & 0 & 0 & 1 & 0 & 2 & 1 & 1 & 0 & 0 & 2 \\
\hline Digestion anaérobie et captation du $\mathrm{CH}_{4}$ & 1 & 0 & 3 & 0 & 1 & 1 & 1 & 0 & 1 & 1 & 1 & 2 & 0 & 0 & 3 \\
\hline Changement d'affectation des terres - total & 0 & 0 & 0 & 0 & 0 & 0 & 0 & 3 & 0 & 0 & 2 & 0 & 0 & 0 & 0 \\
\hline Efficacité énergétique - total & 0 & 0 & 0 & 0 & 0 & 0 & 0 & 0 & 0 & 0 & 2 & 0 & 0 & 15 & 0 \\
\hline Transport & 0 & 0 & 0 & 0 & 0 & 0 & 0 & 0 & 0 & 0 & 0 & 0 & 0 & 1 & 0 \\
\hline Chauffage des locaux & 0 & 0 & 0 & 0 & 0 & 0 & 0 & 0 & 0 & 0 & 0 & 0 & 0 & 7 & 0 \\
\hline Déchets & 0 & 0 & 0 & 0 & 0 & 0 & 0 & 0 & 0 & 0 & 0 & 0 & 0 & 0 & 0 \\
\hline Électricité & 0 & 0 & 0 & 0 & 0 & 0 & 0 & 0 & 0 & 0 & 0 & 0 & 0 & 3 & 0 \\
\hline Chauffage industriel & 0 & 0 & 0 & 0 & 0 & 0 & 0 & 0 & 0 & 0 & 0 & 0 & 0 & 1 & 0 \\
\hline Production d'électricité & 0 & 0 & 0 & 0 & 0 & 0 & 0 & 0 & 0 & 0 & 1 & 0 & 0 & 2 & 0 \\
\hline Efficience des autres consommations d'énergie & 0 & 0 & 0 & 0 & 0 & 0 & 0 & 0 & 0 & 0 & 1 & 0 & & 1 & 0 \\
\hline
\end{tabular}




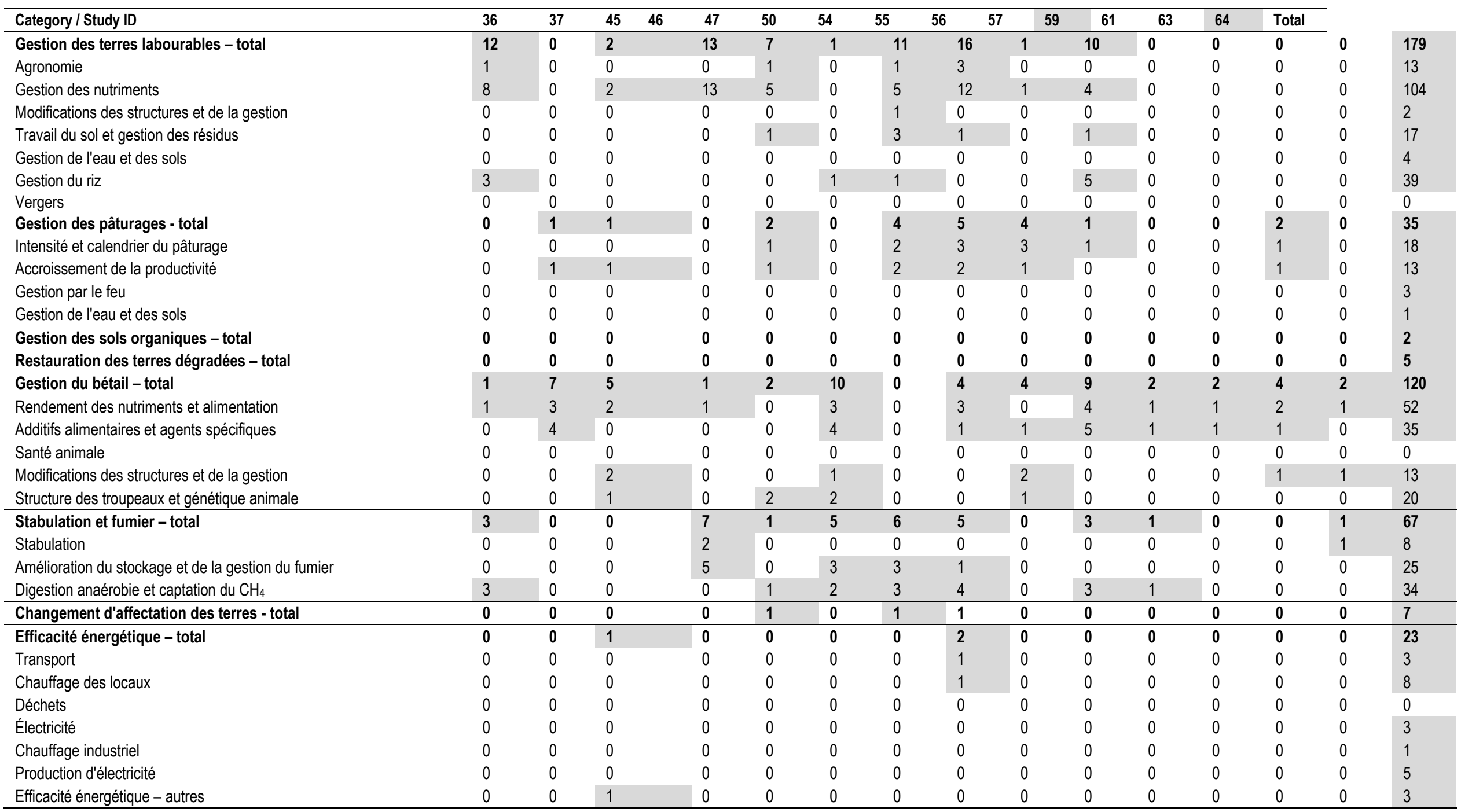


Tableau 29. Nombre de mesures d'atténuation dans chaque catégorie (en gras) et sous-catégorie (en italique)

Sous-catégories

Gestion des terres labourables - total

Agronomie - total

Gestion des nutriments - total

Modifications des structures et de la gestion à plus long terme

Travail du sol et gestion des résidus - total

Gestion de l'eau et des sols - total

Gestion du riz - total

Gestion des pâturages - total

Intensité et calendrier du pâturage - total

Accroissement de la productivité - total

Gestion de l'eau et des sols - total

Gestion des sols organiques - total

Restauration des terres dégradées - total

En général

Amendements nutritifs

Amendements organiques (fumiers, biosolides, composts, etc.)

Diminution du travail du sol

Gestion du bétail - total

Rendement des nutriments et alimentation - total

Additifs alimentaires et agents spécifiques - total

Amélioration génétique animale et structure des troupeaux - total

Stabulation et fumier - total

Stabulation - total

Amélioration du stockage et de la gestion du fumier - total

Digestion anaérobie et captation du $\mathrm{CH}_{4}$ - total

Changement d'affectation des terres - total

Brise-vent/zones ripicoles/bandes tampons

Conversion des terres labourables en pâturages

Boisement

Non conversion des surfaces boisées

Récoltes de biomasse

Sylvopastoralisme

Autres

Efficacité énergétique - total

Transport - total

Chauffage des locaux - total

Électricité - total

Chauffage industriel - total

Production d'électricité - total

Nombre de mesures

\section{4}

7

32

2

6

15

16

17

22

10

24

7

12

5

7

1

1

1

1

1

1

16

1

7

3

1

4 
Tableau 30. Nombre de cas où chaque mesure apparaît dans les études analysées

\begin{tabular}{lcl}
\hline Catégorie/Activité d'atténuation & TOTAL
\end{tabular}

Gestion des terres labourables - total 179

Agronomie - total

Variétés végétales améliorées

Allongement de la phase de culture pérenne dans les rotations culturales

Diminution de la jachère complète

Ajout de nutriments si leur taux est insuffisant

Adoption de systèmes moins dépendants vis-à-vis des intrants (nutriments, pesticides, etc.)

Cultures de couverture/cultures dérobées (vignobles et vergers compris)

Maintien du $\mathrm{pH}$ optimal pour la croissance des végétaux (par chaulage, notamment)

Évolution de cultivars d'hiver vers des cultivars de printemps

0

Agroforesterie (à faible densité de peuplement)

1

0

Haies

Culture des terres afin d'enrichir le sol en carbone (plantes permanentes ou à racines profondes)

Hausse de la production par optimisation des facteurs de production afin d'enrichir le sol en carbone

Agronomie - autres

Gestion des nutriments - total

Agriculture de précision

Éviter l'excès d'azote (par ajustement des objectifs de productivité)

Recours à un système de recommandation relatif aux engrais

Analyse du fumier avant épandage

Prise en compte de l'apport total en azote du fumier

Optimisation du calendrier d'épandage des engrais azotés minéraux (en fonction des conditions météorologiques)

Report du premier épandage d'engrais au printemps

Fractionnement de la fertilisation (maintien de la quantité de départ d'engrais azotés, mais fractionnement en trois apports inférieurs)

Utilisation de la forme appropriée d'engrais azoté minéral

0

Amélioration du calendrier de l'épandage de lisier et de fientes de volaille (en fonction des conditions

météorologiques)

Amélioration du calendrier de l'épandage de fumier (hausse du stockage)

Hausse de l'épandage de fumier au printemps et baisse de l'épandage de fumier en été

Épandage d'engrais banni des zones à haut risque

Respect d'un écart de plusieurs jours entre l'épandage de lisier et l'épandage d'engrais

Utilisation de compost et de fumier pailleux de préférence au lisier

Mélange de résidus de récolte riches en azote avec d'autres résidus de ratio C:N plus élevé

Application précise de l'azote dans le sol

Rampe d'épandage - recommandée pour les terres labourables et les pâturages

Enfouisseur - recommandé principalement pour les pâturages

Injection - recommandée principalement pour les pâturages

Application de fumier après épandage

Épandage de lisier sur de courtes trajectoires

Augmentation du taux d'infiltration dans le sol (dilution du fumier, application d'eau après épandage)

Engrais à libération contrôlée

Production d'inhibiteurs de nitrification naturels par les plantes 0

Épandage d'inhibiteurs d'uréase 0

Maintien du $\mathrm{pH}$ au-dessus de 6.5

Obtentions végétales à assimilation de l'azote renforcée 1

Amélioration génétique de l'efficacité de l'absorption et de l'utilisation de l'azote par les plantes

Fixation biologique de l'azote (légumineuses dans les rotations) 
Baisse de l'utilisation d'engrais azotés

Épandage d'intrants organiques sur les terres labourables au lieu des pâturages

Amélioration de l'entretien des disperseurs d'engrais

Modification des communautés microbiennes du sol afin de réduire le $\mathrm{N}_{2} \mathrm{O}$ en $\mathrm{N}_{2}$ (introduction de souches de

rhizobiums, par exemple)

Zones sans engrais sur les bords des champs

Optimisation de la géométrie de la distribution d'engrais

Bandes tampons autour des plans d'eau

Réduction du chaulage

Gestion des nutriments - autres

Modifications des structures et de la gestion - total

Resserrement des cycles de l'azote (production animale et végétale optimisée à l'échelon régional)

Déplacement des cultures très consommatrices d'azote vers des zones plus sèches, plus fraîches

Diminution de la monoculture

Autres

Travail du sol et gestion des résidus - total

Diminution du travail du sol

Absence de travail du sol

Maintien des résidus de récolte

Éviter l'absence de travail du sol, envisager le labour profond occasionnel

Labourer au début du printemps, épandre uniformément les résidus de récolte et contrôler le tassement du sol 0

Éviter le brûlage des résidus

Ensevelir le biocharbon

Contrôle du trafic

Autres

Gestion de l'eau et des sols - total

Renforcement de l'irrigation

Drainage des sols

Réduire/empêcher le tassement des sols

Empêcher l'érosion du sol

Gestion de l'eau et des sols - autres

Gestion du riz - total

NA

Aération du sol des rizières (réduire leur profondeur, les vider plusieurs fois par an)

Inondation superficielle

Drainage à la mi-saison

Paille hors-saison

Inondation alternée

Compost de paille de riz

Phosphogypse

Semis direct sur sol humide

Remplacement de l'urée par AN

Remplacement de l'urée par du bicarbonate d'ammonium et du sulfate d'ammonium

Modification du type d'engrais (en général)

Autres usages de la paille de riz

Cultivars à faible taux de méthane

Inondation permanente

Riz - autres

Vergers - total

Conversion au système de treillage 
Autres

Gestion des pâturages - tota

0

Intensité et calendrier du pâturage - total

34

Réduction de l'azote sur les pâturages temporaires et permanents les plus intensifs

Hausse du chargement sur les pâturages à productivité moyenne

Pâturage intensif (rotation fréquente du bétail sur les pâturages)

Allongement de la période de pâturage

Retrait du bétail des sols humides

Réduction du chargement

Autres

Accroissement de la productivité - total

Fertilisation

Régénération des pâturages

Éviter l'épandage d'engrais avant la régénération des pâturages

Fixation biologique de l'azote (mélanges cultures herbagères-légumineuses)

Introduction d'espèces ( $\mathrm{y}$ compris de légumineuses)

Nouvelles variétés végétales de fourrage aux caractéristiques nutritionnelles améliorées

Nouvelles variétés végétales de fourrage visant à protéger la croissance des graminées

Amélioration de la digestibilité (qualité du fourrage)

Remplacement de l'ensilage de graminées par de l'ensilage de maïs

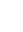

Augmentation de l'ensilage de légumineuses et diminution de l'ensilage de graminées

Graminées à teneur en sucre plus élevée 2

$\begin{array}{ll}\text { Autres } & 2\end{array}$

Allongement de la durée de vie des pâturages temporaires $\quad 1$

$\begin{array}{ll}\text { Accroissement de la productivité des pâturages } & 1\end{array}$

Renforcement de l'irrigation $\quad 1$

Gestion par le feu - total $\quad 2$

Gestion par le feu
Gestion de l'eau et des sols - total

Gestion de l'eau et des sols - total $\quad 1$

Ajuste le $\mathrm{pH}$ au-dessus de 5 par chaulage $\quad 1$

Drainage des sols - VOIR GESTION DES TERRES LABOURABLES 0

Empêcher le tassement du sol - VOIR GESTION DES TERRES LABOURABLES 0

$\begin{array}{ll}\text { Gestion des sols organiques - total } & \mathbf{2}\end{array}$

Éviter le drainage des terres humides/la conversion des tourbières 1

$\begin{array}{ll}\text { Éviter les cultures sarclées et de tubercules } & 0\end{array}$

$\begin{array}{ll}\text { Éviter le labour profond } & 0\end{array}$

$\begin{array}{ll}\text { Maintenir une nappe d'eau superficielle - tourbières } & 0\end{array}$

$\begin{array}{ll}\text { Maintenir une nappe d'eau superficielle - terres labourables } & 0\end{array}$

$\begin{array}{ll}\text { Autres } & 1\end{array}$

\begin{tabular}{lc}
\hline Restauration des terres dégradées - total & 5 \\
En général & 1 \\
Maîtrise de l'érosion & 0 \\
Restauration du couvert végétal & 0 \\
Amendements nutritifs & 1 \\
Amendements organiques (fumiers, biosolides, composts, etc.) & 1 \\
Allègement du travail du sol & 1 \\
Conservation des résidus de récolte & 0 \\
Conservation de l'eau & 0 \\
Autres & 1 \\
\hline
\end{tabular}

$\begin{array}{lr}\text { Gestion du bétail - total } & 120\end{array}$ 
Rendement des nutriments et alimentation - total

52

Augmentation de la part de concentrés dans l'alimentation (en remplacement des fourrages)

Besoins du bétail en nutriments fixés en fonction d'objectifs spécifiques

Alimentation riche en lipides (matières grasses alimentaires)

Alimentation équilibrée entre apport d'énergie et apport de protéines (réduction de la part de protéines,

augmentation de la part de glucides

Alimentation riche en amidon

Alimentation riche en amidon (maïs)

Amélioration de la qualité des protéines (composition équilibrée en acides aminés essentiels)

Optimiser (= réduire) l'ingestion de protéines du bétail

Réduire l'ingestion de protéines (sans complémentation en acides aminés)

Réduire l'ingestion de protéines et fournir une complémentation en acides aminés

Qualité supérieure de l'avoine

Augmentation de la fréquence de traite

Somatotropine bovine

Estimation de la production potentielle de $\mathrm{CH}_{4}$ à partir des aliments du bétail

Transformation des aliments du bétail

Traitement mécanique des aliments du bétail

Traitement chimique des aliments de faible qualité

Alimentation à base de rations totales mixtes

Alimentation de précision (+ analyse des aliments)

Alimentation par phases (multiples)

Amélioration du coefficient de conversion des aliments (hausse de la valeur énergétique et de la digestibilité)

Poursuite de l'amélioration conventionnelle de l'alimentation

Amélioration de l'alimentation des porcins

Réduire l'excès de graisse sous-cutanée des bœufs et des agneaux à mesure qu'ils approchent de leur poids à

l'abattage

Accroître la masse graisseuse pour l'abattage

Aliments du bétail dérivés de l'industrie alimentaire

Aliments du bétail produits sur l'exploitation

Autres

Amélioration de la prise alimentaire

Additifs alimentaires et agents spécifiques - total

En général

Augmentation de la teneur en sel de l'alimentation

Extraits végétaux

Huiles essentielles

Tanins

Saponines

lonophores (monensin, par exemple)

Antibiotiques

Précurseurs d'acide propionique

Nitrate

Administration directe d'inhibiteurs de nitrification

Séparation des hexoses

Administration directe de micro-organismes probiotiques (levures, notamment)

Manipulation des archébactéries et des bactéries du rumen

Administration directe de micro-organismes - bactéries acétogènes, oxydants du méthane

Promotion des bactéries acétogènes

Anti-méthanogènes

(1)


Modification génétique de la microflore du rumen $\quad 0$

Vaccination contre les bactéries méthanogènes 3

Accepteurs alternatifs d'hydrogène (acides gras non saturés, par exemple)

Analogues halogénés du méthane $\quad 0$

$\begin{array}{lc}\text { Agents de défaunation } & 0\end{array}$

Composés végétaux spontanés (nouvelles espèces/transformations génétiques) 0

Chicorée $\quad 0$

$\begin{array}{lr}\text { Utilisation d'enzymes exogènes } & 0\end{array}$

Allicine $\quad 1$

Glycérol $\quad 1$

Autres $\quad 1$

$\begin{array}{lr}\text { Santé animale - total } & 0\end{array}$

$\begin{array}{ll}\text { Meilleure planification sanitaire } & 0\end{array}$

Amélioration de l'hygiène et de la supervision lors de l'agnelage $\quad 0$

Amélioration de la nutrition des brebis en fin de gestation afin d'accroître les chances de survie de l'agneau 0

Vaccination $\quad 0$

$\begin{array}{lr}\text { Antiparasites } & 0\end{array}$

$\begin{array}{ll}\text { Autres } & 0\end{array}$

Modifications des structures et de la gestion - total 13

Réduction du nombre de génisses/Amélioration de la gestion de la fertilité 2

Polyvalence des vaches (lait, veaux et viande) $\quad 0$

Augmentation de la production d'aliments du bétail à l'échelle de l'exploitation ou à l'échelle locale 0

Agriculture biologique $\quad 0$

Gestion hivernale du bétail (collecte et réutilisation des excrétats) $\quad 0$

Hausse de la part du pâturage par rapport à la stabulation 1

Hausse de la part de la stabulation par rapport au pâturage 1

Réduction des taux de charge $\quad 6$

Contournement de la phase de la jeune bête destinée à l'engraissement 1

Autres

Amélioration génétique animale et structure des troupeaux - total $\quad 20$

Sélection visant à réduire la production de méthane $\quad 5$

Sélection visant à renforcer la longévité, la fertilité et autres caractéristiques étrangères à la production 1

Sélection visant à accroître le rendement/gain de poids 3

Sélection visant à réduire les émissions de $\mathrm{CH}_{4}$ (ingestion résiduelle) 0

Amélioration du rendement laitier de $30 \%$ - bétail laitier 0

Amélioration du rendement laitier 2

Amélioration du coefficient de conversion des aliments $\quad 0$

Clonage $\quad 0$

$\begin{array}{lr}\text { Bétail génétiquement modifié } & 0\end{array}$

$\begin{array}{ll}\text { Insémination artificielle } & 0\end{array}$

Sélection planifiée du sexe avant insémination (sexage des embryons et du sperme) 0

$\begin{array}{ll}\text { Jumelage } & 0\end{array}$

Manipulation transgénique $\quad 2$

Amélioration du potentiel génétique en général $\quad 3$

Amélioration du potentiel génétique - productivité 1

Amélioration du potentiel génétique - fertilité $\quad 1$

Amélioration du potentiel génétique - longévité $\quad 0$ 


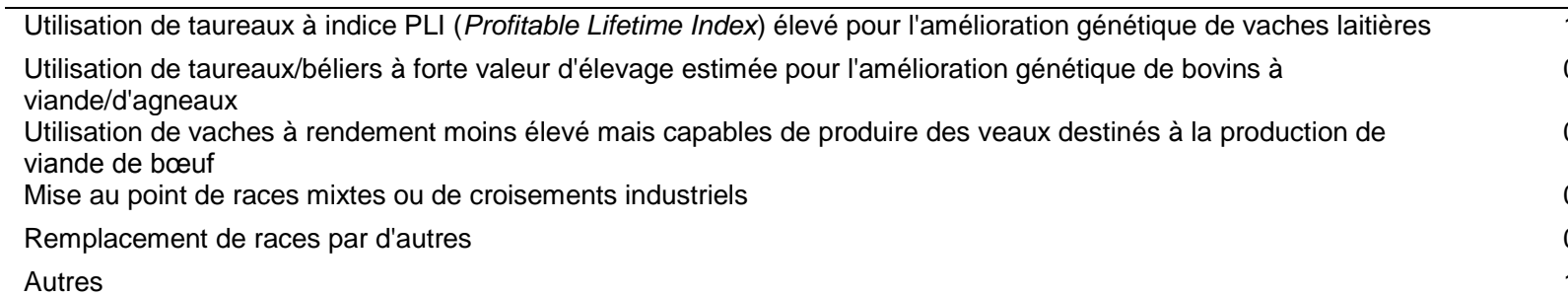

Stabulation et fumier - total 67

Stabulation - total

Nouveaux systèmes de stabulation à faibles émissions pour le bétail et la volaille

Ventilation naturelle

Ralentissement de la vitesse de l'air au-dessus du fumier

0

0

0

1

Refroidissement des surfaces recouvertes de fumier

Filtration des émissions provenant des locaux de stabulation

Systèmes de stabulation entravée au lieu de stabulation libre

Systèmes sur lisier - bétail laitier

Systèmes paillés - bétail laitier

Stabulation libre, systèmes de litière montée - bétail laitier

Cages et volières au lieu de systèmes au sol pour les poules pondeuses 0

Maintien des surfaces, du fumier et des animaux au sec 0

Amélioration des systèmes d'abreuvoirs 0

Séchage du fumier (en particulier des fientes de volaille) 0

Absorption de l'urine/Utilisation de litière 0

Systèmes paillés 0

Systèmes de litière montée 0

Systèmes de litière montée - porcins 0

Systèmes sur lisier/Rigoles profondes de drainage des déjections 0

Sol partiellement ou intégralement recouverts de caillebotis 0

Enlèvement fréquent du fumier et transfert vers un stockage en extérieur (plus froid) 1

Type d'étable 1

Autres 2

Amélioration du stockage et de la gestion du fumier - total 25

Refroidissement

Ralentissement de la décomposition anaérobie par retrait du lisier vers un stockage en extérieur - porcins 0

Réduction du flux d'air au-dessus du lisier et du fumier de ferme 0

Couverture des fumières 0

Abaissement du taux de remplissage des cuves à lisier 0

Couverture du lisier (huile, plastique, paille, granulés, couverture rigide, par exemple) 7

Semi-perméable 1

Imperméable 2

Permettre la constitution d'une croûte naturelle au-dessus du lisier du bétail 2

Séparer les éléments solides du lisier 1

Séparation rapide des matières fécales et de l'urine 0

Compostage du fumier solide (également après séparation du lisier) 0

Remplacement de l'utilisation de fumier solide par un procédé (anaérobie) employant le lisier 1

Remplacement des installations anaérobies par des installations aérobies 2

Aération maîtrisée pendant le stockage du lisier 0

Réduction au minimum de l'agitation du lisier 0

Acidification du fumier 0 


\begin{tabular}{|c|c|}
\hline Réduction de la surface du lisier ou du fumier de ferme par unité de volume (réservoirs au lieu de bassins) & 0 \\
\hline Combustion de la litière des volailles & 0 \\
\hline Maîtrise des processus de dénitrification du lisier & 0 \\
\hline Hausse de la teneur en carbone du fumier (par ajout de paille) & 1 \\
\hline Compactage du fumier de ferme & 0 \\
\hline Broyage du fumier de ferme & 0 \\
\hline Augmentation de la fréquence de l'épandage de lisier & 0 \\
\hline Séchage des fientes de volaille dans l'exploitation & 0 \\
\hline Incinération de la litière des volailles & 1 \\
\hline Épandage quotidien du fumier & 2 \\
\hline Autres & 4 \\
\hline Digestion anaérobie et captation du $\mathrm{CH}_{4}-$ total & 34 \\
\hline Digestion anaérobie & 18 \\
\hline Digestion anaérobie centralisée & 3 \\
\hline Digestion anaérobie sur l'exploitation & 4 \\
\hline Méthanisation, captation du méthane et combustion & 6 \\
\hline Captation du méthane par flambage (sans production d'énergie) & 0 \\
\hline Production de $\mathrm{H}_{2}$ à partir du fumier par un processus anaérobie et conversion en énergie & 0 \\
\hline Autres & 1 \\
\hline Changement d'affectation des terres - total & 7 \\
\hline Brise-vent/zones ripicoles/bandes tampons & 1 \\
\hline Conversion des terres labourables en pâturages & 1 \\
\hline Boisement des terres labourables & 0 \\
\hline Mise hors production des terres labourables & 0 \\
\hline Boisement des pâturages & 0 \\
\hline Terres labourables drainées - terres humides & 0 \\
\hline Boisement & 1 \\
\hline Restauration permanente du couvert végétal des terres mises hors production & 0 \\
\hline Rétablissement des tourbières & 0 \\
\hline Non conversion des surfaces boisées & 1 \\
\hline Récoltes de biomasse & 1 \\
\hline Sylvopastoralisme & 1 \\
\hline Autres & 1 \\
\hline Efficacité énergétique - total & 23 \\
\hline Transport - total & 3 \\
\hline Réduction de la consommation de carburant des tracteurs, etc. & 3 \\
\hline Équiper et entretenir les véhicules (pression des pneus, niveaux d'huile, etc.) & 0 \\
\hline Veiller à l'emploi de techniques de conduite favorisant l'économie de carburant par les employés & 0 \\
\hline Transport - autres & 0 \\
\hline Chauffage des locaux - total & 8 \\
\hline Micro-dispositif de cogénération & 1 \\
\hline Amélioration de l'efficience du chauffage (stabulations et serres) & 2 \\
\hline Ajustement des ferme-porte automatiques, des portes souples, des bandes d'étanchéité & 1 \\
\hline Utilisation de brise-vent afin de protéger contre les vents violents & 0 \\
\hline Amélioration de l'isolation (toits, murs, tuyaux chauds intérieurs et extérieurs) & 1 \\
\hline $\begin{array}{l}\text { Amélioration du chauffage (installation de minuteurs et de thermostats, commandes distinctes pour le chauffage et la } \\
\text { ventilation) }\end{array}$ & 1 \\
\hline Entretenir et vérifier régulièrement les thermostats & 0 \\
\hline
\end{tabular}




\begin{tabular}{|c|c|}
\hline Rediffuser l'air chaud dans les bâtiments hauts de l'exploitation & 0 \\
\hline Réduire la ventilation au minimum, en particulier lorsque le chauffage fonctionne. & 0 \\
\hline Utiliser de l'air extérieur pour le refroidissement dès que possible. & 0 \\
\hline Vérifications régulières de la ventilation (nettoyage, entretien) & 0 \\
\hline Ajuster la ventilation à vitesse variable & 0 \\
\hline Entretenir régulièrement les chaudières & 0 \\
\hline Déplacer les serres chauffées vers des zones où elles peuvent tirer profit de la chaleur produite par les déchets & 0 \\
\hline Nettoyer régulièrement les vitres des serres afin de mieux tirer profit de la chaleur solaire & 0 \\
\hline Installer des écrans thermiques dans les serres & 1 \\
\hline Remplacer les carreaux brisés ou tombés & 0 \\
\hline Chauffage des locaux - autres & 1 \\
\hline Déchets - total & 0 \\
\hline Réduction des déchets pendant le transport, la transformation et le stockage & 0 \\
\hline Déchets - autres & 0 \\
\hline Électricité - total & 3 \\
\hline Pré-refroidissement du lait par un refroidisseur à plaques lait-eau & 1 \\
\hline Réfrigération efficiente (loin des sources de chaleur, dégivrage régulier, entretien) & 1 \\
\hline Lait - autres & 0 \\
\hline Utilisation de lampes fluorescentes & 0 \\
\hline Utilisation de tubes fluorescents plus efficients & 0 \\
\hline Éclairage - autres & 1 \\
\hline Écourter le temps de lavage à pression par un trempage préalable & 0 \\
\hline Autres & 0 \\
\hline Transformation par chauffage - total & 1 \\
\hline Séchage amélioré de la production & 1 \\
\hline Entretien et nettoyage régulier des séchoirs & 0 \\
\hline Utilisation de capteurs d'humidité lors du séchage & 0 \\
\hline Planifier le séchage de manière à tirer le meilleur parti des périodes les moins humides de l'année & 0 \\
\hline Transformation par séchage & 0 \\
\hline Production d'électricité - total & 5 \\
\hline Utilisation d'électricité renouvelable & 0 \\
\hline Combustion de la biomasse & 3 \\
\hline Énergie solaire & 1 \\
\hline Énergie éolienne & 0 \\
\hline Chauffage solaire de l'eau & 0 \\
\hline Énergie hydraulique à petite échelle & 0 \\
\hline Pompes à chaleur géothermiques ou aérothermiques & 1 \\
\hline Production électrique - autres & 0 \\
\hline Efficacité énergétique - autres & 3 \\
\hline
\end{tabular}

